\title{
Reduced classes and curve counting on surfaces I: theory
}

\author{
Martijn Kool and Richard Thomas \\ With an appendix written with Dmitri Panov
}

\begin{abstract}
We develop a theory of reduced Gromov-Witten and stable pair invariants of surfaces and their canonical bundles.

We show that classical Severi degrees are special cases of these invariants. This proves a special case of the MNOP conjecture, and allows us to generalise the Göttsche conjecture to the non-ample case. In a sequel we prove this generalisation.

We prove a remarkable property of the moduli space of stable pairs on a surface. It is the zero locus of a section of a bundle on a smooth compact ambient space, making calculation with the reduced virtual cycle possible.
\end{abstract}

\section{Contents}

1 Introduction $\quad 335$

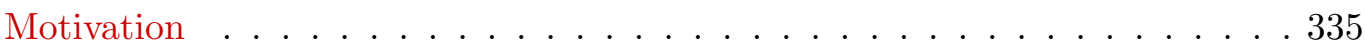

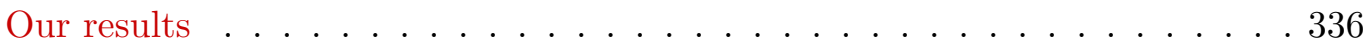

Notation . . . . . . . . . . . . . . . . . . . . . 339

2 Reduced obstruction theories $\quad 340$

2.1 The algebraic twistor family $\mathcal{S}_{B} \ldots \ldots \ldots$. . . . . . . . . . 340

2.2 Stable maps . . . . . . . . . . . . . . . . . . . . . . . . . . . . . . . . . . . . . . . . . . . . . . . . . . . . .

2.3 Stable pairs . . . . . . . . . . . . . . . . . . . 347

3 Invariants $\quad 349$

3.1 Reduced Gromov-Witten invariants . . . . . . . . . . . . . . . . . . 349

3.2 Reduced stable pair invariants . . . . . . . . . . . . . . . . 352

4 Insertions and linear systems $\quad 357$

4.1 Det and $\operatorname{div} \ldots \ldots \ldots \ldots \ldots \ldots$. . . . . . . . . . . . . . . . . . . . . . . .

4.2 Point insertions and linear subsystems . . . . . . . . . . . . 360

4.3 Extension to pairs and the threefold $X \ldots \ldots$. . . . . . . . 361

5 Counting nodal curves $\quad \mathbf{3 6 2}$

5.1 Severi degrees as reduced Gromov-Witten invariants . . . . . . . . . . . 362

Received 12 November 2013, accepted in final form 13 February 2014.

2010 Mathematics Subject Classification 14N35 (primary), 14C05 (secondary)

Keywords: Gromov-Witten invariants, stable pair invariants, reduced Gromov-Witten invariants, perfect obstruction theory, surfaces, Gttsche conjecture.

This journal is (C) Foundation Compositio Mathematica 2014. This article is distributed with Open Access under the terms of the Creative Commons Attribution Non-Commercial License, which permits non-commercial reuse, distribution, and reproduction in any medium, provided that the original work is properly cited. For commercial re-use, please contact the Foundation Compositio Mathematica.

Both authors were supported by an EPSRC programme grant EP/G06170X/1. 


\section{Curve COUnTing ON SURFACES I: THEORY}

5.2 Severi degrees as reduced stable pair invariants . . . . . . . . . . . 365

Appendix A. Reduced obstruction theory for stable pairs revisited written with Dmitri Panov $\quad \mathbf{3 6 9}$

A.1 The Hilbert scheme of curves as a zero locus . . . . . . . . . . . . . . . . 369

A.2 The relative Hilbert scheme of points as a zero locus . . . . . . . . . . . 373

A.3 Identifying the obstruction theories . . . . . . . . . . . . . 373

References

\section{Introduction}

\section{Motivation}

Fix a nonsingular projective surface $S$ and a homology class $\beta \in H_{2}(S, \mathbb{Z})$. There are various ways of counting holomorphic curves in $S$ in class $\beta$; in this paper we focus on Gromov-Witten invariants [Beh97, LT98] and stable pairs [PT09]. Since these are deformation invariant they must vanish in class $\beta$ if there exists a deformation of $S$ for which the Hodge type of $\beta$ is not $(1,1)$. We can see the origin of this vanishing without deforming $S$ as follows.

For simplicity work in the simplest case of an embedded curve $C \subset S$ with normal bundle $N_{C}=\mathcal{O}_{C}(C)$. As a Cartier divisor, $C$ is the zero locus of a section $s_{C}$ of a line bundle $L:=\mathcal{O}_{S}(C)$, giving the exact sequence

$$
0 \longrightarrow \mathcal{O}_{S} \stackrel{s_{C}}{\longrightarrow} L \longrightarrow N_{C} \longrightarrow 0
$$

The resulting long exact sequence describes the relationship between first order deformations and obstructions $H^{0}\left(N_{C}\right), H^{1}\left(N_{C}\right)$ of $C \subset S$, and the deformations and obstructions $H^{1}\left(\mathcal{O}_{S}\right)$, $H^{2}\left(\mathcal{O}_{S}\right)$ of the line bundle $L \rightarrow S$ :

$$
\begin{aligned}
0 \longrightarrow H^{0}(L) /\left\langle s_{C}\right\rangle & \longrightarrow H^{0}\left(N_{C}\right) \longrightarrow H^{1}\left(\mathcal{O}_{S}\right) \longrightarrow H^{1}(L) \\
& \longrightarrow H^{1}\left(N_{C}\right) \longrightarrow H^{2}\left(\mathcal{O}_{S}\right) \longrightarrow H^{2}(L) \longrightarrow 0 .
\end{aligned}
$$

The resulting "semi-regularity map" [KS59] $H^{1}\left(N_{C}\right) \rightarrow H^{2}\left(\mathcal{O}_{S}\right)=H^{0,2}(S)$ takes obstructions to deforming $C$ to the "cohomological part" of these obstructions. Roughly speaking, if we deform $S$, we get an associated obstruction in $H^{1}\left(N_{C}\right)$ to deforming $C$ with it; its image in $H^{0,2}(S)$ is the $(0,2)$-part of the cohomology class $\beta \in H^{2}(S)$ in the deformed complex structure. Thus it gives the obvious cohomological obstruction to deforming $C$ : that $\beta$ must remain of type $(1,1)$ in the deformed complex structure on $S$.

In particular, when $S$ is fixed, obstructions lie in the kernel of $H^{1}\left(N_{C}\right) \rightarrow H^{2}\left(\mathcal{O}_{S}\right)$. More generally, if we only consider deformations of $S$ for which $\beta$ remains $(1,1)$ then the same is true. And when $h^{0,2}(S)>0$ but $H^{2}(L)=0$, the existence of this trivial $H^{0,2}(S)$ piece of the obstruction sheaf guarantees that the virtual class vanishes.

So it would be nice to restrict attention to surfaces and classes $(S, \beta)$ inside the NoetherLefschetz locus (the locus of surfaces $S$ for which $\beta \in H^{2}(S)$ has type $(1,1)$; for more details see [Voi13, MP13]) defining a new obstruction theory using only the kernel of the semi-regularity map. ${ }^{1}$ Checking that this kernel really defines an obstruction theory in the generality needed to define a virtual cycle - that is, for deformations to all orders, over an arbitrary base, of possibly non-embedded curves - has proved difficult; there is a hotchpotch of results in different cases

\footnotetext{
${ }^{1}$ For embedded curves, this means we use the obstruction space $H^{1}(L)$ to deforming sections of $L$. We have been able to remove the obstructions $H^{2}\left(\mathcal{O}_{S}\right)$ to deforming $L$ since the space of line bundles is smooth over the Noether-Lefschetz locus.
} 
[BeF98, BL00, Blo72, BuF03, Don90, IM13, KL07, Lee04, Li02, Liu00, Man07, MP13, MPT10, OP10, Ran99, Ros12, Sch12, STV13]. Here we give quite a general construction using a mixture of some of these methods. Since this paper appeared, Jon Pridham has found a more general solution [Pri12] using derived deformation theory, $\infty$-stacks, etc. His result is broader and more natural, but our methods are much more elementary.

\section{Our results}

Surfaces. For stable pairs on $S$ we get optimal results. We show that the kernel of the semiregularity map gives a reduced perfect obstruction theory, virtual cycle and invariants whenever

$$
H^{2}(L)=0 \text { for effective line bundles } L \text { with } c_{1}(L)=\beta .
$$

Equivalently, by Serre duality, the condition is that there is no curve in class $\beta$ which is contained in a canonical divisor of $S$. This condition is necessary to ensure the semi-regularity map (1) is surjective.

For Gromov-Witten theory, multiple covers complicate the situation, but we are able to prove the same result for the moduli space of stable maps when

$$
H^{1}\left(T_{S}\right) \stackrel{\cup \beta}{\longrightarrow} H^{2}\left(\mathcal{O}_{S}\right) \text { is surjective. }
$$

Here $\beta \in H^{1}\left(\Omega_{S}\right)$ and we use the pairing $\Omega_{S} \otimes T_{S} \rightarrow \mathcal{O}_{S}$. Condition (3) implies (2): for any $L=\mathcal{O}(C)$ in class $\beta$, the map $\cup \beta$ factors through

$$
H^{1}\left(T_{S}\right) \longrightarrow H^{1}\left(\mathcal{O}_{C}(C)\right) \longrightarrow H^{2}\left(\mathcal{O}_{S}\right)
$$

so surjectivity implies that $H^{2}(L)=0$ by the exact sequence (1).

Condition (3) is a transversality assumption on the moduli space of surfaces $S$. It asks that the $h^{2,0}(S)$ equations

$$
\int_{\beta} \sigma_{i}=0, \quad\left\{\sigma_{i}: i=1, \ldots, h^{2,0}(S)\right\} \text { a basis for } H^{2,0}(S)
$$

cutting out the Noether-Lefschetz locus are transverse to 0 . In particular if the moduli space of surfaces is smooth, it asks that the Noether-Lefschetz locus be smooth of the expected codimension $h^{2,0}(S)$. For example, we note that for degree $d \geqslant 4$ surfaces $S \subset \mathbb{P}^{3}$, it is almost always satisfied in a precise sense [Kim91, Section 3].

Method. We embed $S$ as the central fibre of an algebraic twistor family ${ }^{2}$

$$
\mathcal{S}_{B} \rightarrow B
$$

Here $B$ is a first order Artinian neighbourhood of the origin in a certain $h^{0,2}(S)$-dimensional family of first order deformations of $S$.

In Section 2 we show that condition (3) implies that the relative moduli space of curves (stable maps or stable pairs) on the fibres of $\mathcal{S}_{B}$ is in fact the moduli space of curves on the central fibre. We then show that the natural perfect obstruction theory of the family (its relative perfect obstruction theory made absolute) is isomorphic to the kernel of the semi-regularity map on the standard obstruction theory. Thus the latter, which is canonical, can indeed be used as

\footnotetext{
${ }^{2}$ This is an outrageous abuse of notation, motivated by the $S=K 3$ case where it is a first order neighbourhood of the central fibre in the twistor family used in [BL00]. It should also be noted that the family is not canonical, but involves choices.
} 


\section{Curve COUNTING ON SURFACES I: THEORY}

an obstruction theory, giving a definition of reduced curve counting invariants. These coincide with the usual invariants when $h^{0,2}(S)=0$.

This use of relative moduli spaces means that we require condition (3) only for $\beta$. When condition (3) holds also for all $\beta^{\prime}<\beta$ then the absolute moduli space of curves in $\mathcal{S}_{B}$ also coincides with the moduli space for $S$, and the ordinary Gromov-Witten invariants of the total space $\mathcal{S}_{B}$ can be expressed in terms of the reduced Gromov-Witten invariants of $S$ with so-called $K_{S}$-twisted $\lambda$-class insertions. (These are Chern classes of the virtual bundle whose fibre over $f: C \rightarrow S \subset \mathcal{S}_{B}$ is $R \Gamma\left(f^{*} K_{S}\right)$. For the K3 case see [MPT10].)

Threefolds. We also work on the Calabi-Yau threefold

$$
\left(X=K_{S}\right) \curvearrowleft \mathbb{C}^{*},
$$

always using condition (3) in this case. Again we get a reduced obstruction theory, and use $\mathbb{C}^{*}$-localisation to define reduced residue Gromov-Witten and stable pair invariants. The former come entirely from the moduli space of stable maps to $S$ itself and include the reduced invariants of $S$. More generally they include some $\lambda$-classes twisted by $K_{S}$. The moduli space $P_{X}^{\mathbb{C}^{*}}$ of stable pairs fixed by the $\mathbb{C}^{*}$-action, however, contains stable pairs not scheme-theoretically supported on $S$, so the moduli space is bigger than the moduli space $P_{S}$ of stable pairs on $S$. But $P_{S} \subset P_{X}^{\mathbb{C}^{*}}$ forms a connected component, so the reduced residue invariants of $X$ contain a contribution coming entirely from $S$. This contribution includes the reduced invariants of $S$, and more generally signed virtual Euler characteristics of loci in $P_{S}$ satisfying incidence conditions. In the sequel [KT14], we describe conditions under which $P_{S}$ is all of $P_{X}^{\mathbb{C}^{*}}$.

Insertions and Severi degrees. In Section 4 we develop a careful treatment of insertions in these theories. We then prove various folklore results about them, such as the link to the Picard variety and sublinear systems. (The existing literature only handles these issues within symplectic geometry.)

In Section 5 we use this to show that with the right insertions, the reduced Gromov-Witten invariants recover the Severi degrees

$$
n_{\delta}(L):=\operatorname{deg} \overline{\{C \in|L|: C \text { has } \delta \text { nodes }\}} \subset|L|
$$

of appropriately ample linear systems $|L|$ on $S$.

In this case we are also able to show that only $P_{S} \subset P_{X}^{\mathbb{C}^{*}}$ contributes to the stable pair invariants of $X$. Therefore the threefold MNOP conjecture [MNOP06] applied to $X$ (and extended to the reduced $\mathbb{C}^{*}$-localised invariants) predicts that the $n_{\delta}(L)$ should also be expressible as a precise combination of reduced stable pair invariants of $S$. We prove this in Theorem 5.4.

This also points to a definition of virtual Severi degrees in the non-ample case via reduced Gromov-Witten or stable pair invariants. Moreover, we give an extension of the Göttsche conjecture to these virtual numbers, that is, that they should be computed by the Göttsche polynomials even when the latter are not obviously enumerative. In the sequel [KT14] we compute the resulting stable pair invariants and prove this version of the conjecture.

Stable pairs as a zero locus. In Appendix 5.2, written with Dmitri Panov, we give an alternative, more direct, construction of the reduced stable pair theory on a surface $S$, without reference to $\mathcal{S}_{B}$. This is achieved by realising the moduli space in the following way. We first take the zero locus of a natural section of a bundle over a smooth ambient space, then we take the 


\section{Kool, R. P. Thomas}

zero locus of a section of another bundle over that.

After posting this paper we discovered that the first step here was used many years ago by Dürr, Kabanov and Okonek [DKO07], giving a description of the virtual cycle on the Hilbert scheme of curves in $S$. We then extend this to stable pairs over those curves.

This is an unusual phenomenon. Having a local description of a moduli space as the zero locus of a section of a bundle $E$ over a smooth ambient space $A$ with

$$
\operatorname{dim} A-\operatorname{rank} E=v
$$

is basically equivalent to having a perfect obstruction theory of virtual dimension $v$. But having such a description globally, for some compact $A$ and the same $v$, is extremely rare. It is also extremely desirable: it means the pushforward to $A$ of the virtual cycle is Poincaré dual to $c_{t o p}(E)$, with which one can try to calculate.

We exploit this in the sequel [KT14] to calculate the reduced stable pair invariants in terms of universal formulae in topological numbers ${ }^{3}$ of $(S, \beta)$. In forthcoming work [PT14], this also provides one of the foundations of a computation of the full stable pairs theory of the twistor family of a K3 surface. Via Pandharipande and Pixton's recent proof of the MNOP conjecture for many threefolds, this then gives a proof of the famous KKV formula for the Gromov-Witten invariants of $\mathrm{K} 3$ surfaces in all genera, degrees and for all multiple covers.

Except for genus zero Gromov-Witten calculations in complete intersections in convex varieties, we know of no other moduli problem where such direct calculation is possible. Usually obtaining explicit results is very complicated, involving various difficult degeneration and localisation tricks.

Organisation. The paper is organised as follows. Most results are proved twice, once for stable maps, and an analogous result for stable pairs. We work out the reduced obstruction theories in Section 2 assuming condition (3). In Appendix 5.2 we use an easier construction to show that condition (3) can be replaced by condition (2) for stable pairs on $S$ only. In Section 3 we define the corresponding reduced invariants for $S$, the reduced residue invariants of $X=K_{S}$, and we show the latter contain the information of the former. Section 4 deals with insertions and linear systems of curves in $S$. Section 5 discusses the application to Severi degrees and the MNOP conjecture for reduced invariants with many point insertions.

In summary, we describe invariants incorporating the following:

- reduced Gromov-Witten and stable pair invariants of $S$

- Gromov-Witten and stable pair invariants of $S$ when $H^{2,0}(S)=0$

- reduced equivariant Gromov-Witten and stable pair invariants of $K_{S}$

- equivariant invariants of $K_{S}$ when $H^{2,0}(S)=0$

- reduced GW invariants of $S$ with $K_{S}$-twisted $\lambda$-class insertions

- relative Gromov-Witten and stable pair invariants of $\mathcal{S}_{B} \rightarrow B$

- absolute Gromov-Witten invariants of $\mathcal{S}_{B}$

- absolute equivariant Gromov-Witten invariants of $K_{\mathcal{S}_{B} / B}$

- Severi degrees and virtual Severi degrees

\footnotetext{
${ }^{3}$ In contrast, the nonreduced stable pair invariants of $S$ do not have such a simple form in general, depending on the Seiberg-Witten invariants of $S$. See [Koo13], where a duality formula is also obtained, and the MNOP conjecture is related to Taubes' $\mathrm{SW}=\mathrm{GW}$ correspondence.
} 


\section{Notation}

Throughout we keep largely to the following notation.

\begin{tabular}{|c|c|}
\hline$S$ & a smooth projective surface \\
\hline$X, \bar{X}$ & $\begin{array}{l}\text { the total space of the canonical bundle } K_{S} \text { of } S \text { and its } \\
\text { projective completion } \mathbb{P}\left(K_{S} \oplus \mathcal{O}_{S}\right) \text { respectively }\end{array}$ \\
\hline$V \supset B$ & $\begin{array}{l}\text { choice }(5) \text { of } h^{0,2}(S) \text {-dimensional subspace of } H^{1}\left(T_{S}\right) \\
\text { and first order thickening (6) of its origin }\end{array}$ \\
\hline $\mathcal{S}_{B}, \mathcal{X}_{B}, \overline{\mathcal{X}}_{B}$ & $\begin{array}{l}\text { (6) the algebraic twistor family of } S \text { over the Artinian } \\
\text { base } B \text {, its relative canonical bundle and completion }\end{array}$ \\
\hline$\iota, q$ & inclusion map of $S$ into $X, \bar{X}$ and projection $q: X, \bar{X} \rightarrow S$ \\
\hline$\beta$ & a class in $H^{2}(S, \mathbb{Z})$, usually of Hodge type $(1,1)$ \\
\hline$h$ & $\begin{array}{l}\text { the arithmetic genus of curves in class } \beta \text {, determined by } \\
\text { the adjunction formula } 2 h-2=\beta^{2}-\int_{\beta} c_{1}(S)\end{array}$ \\
\hline $\begin{array}{l}L \\
\text { condition }(2)\end{array}$ & $\begin{array}{l}\text { a line bundle on } S \text { with } c_{1}(L)=\beta \\
H^{2}(L)=0 \text { for effective line bundles } L \text { with } c_{1}(L)=\beta\end{array}$ \\
\hline condition (3) & $H^{1}\left(T_{S}\right) \stackrel{\cup \beta}{\longrightarrow} H^{2}\left(\mathcal{O}_{S}\right)$ is surjective \\
\hline$\gamma_{i}$ & (49) integral basis $\gamma_{1}, \ldots, \gamma_{b_{1}(S)}$ of $H_{1}(S, \mathbb{Z}) /$ torsion, \\
\hline $\operatorname{div}$ & (46) divisor class in $S$ of a stable map \\
\hline $\operatorname{det}$ & (46) line bundle associated to above divisor class \\
\hline$[\gamma]$ & Poincaré dual of homology class $\gamma$ \\
\hline $\mathcal{P}$ & Poincaré line bundle on $S \times \operatorname{Pic}(S)$ \\
\hline $\mathfrak{t}, t=c_{1}(\mathfrak{t})$ & $\begin{array}{l}\text { one dimensional irreducible representation of } T=\mathbb{C}^{*} \text { of } \\
\text { weight one, and generator of } H^{*}\left(B \mathbb{C}^{*}, \mathbb{Z}\right)=\mathbb{Z}[t] \text { respectively }\end{array}$ \\
\hline $\bar{M}_{g, n}(S, \beta)$ & $\begin{array}{l}\text { moduli space of stable maps from connected genus } g \\
\text { curves with } n \text { marked points to } S \text { in class } \beta\end{array}$ \\
\hline $\bar{M}_{g}(S, \beta)$ & as above but without marked points, that is, $n=0$ \\
\hline $\bar{M}_{g, n}\left(S, \mathbb{P}^{\delta}\right)$ & $\begin{array}{l}\text { (51) stable maps whose divisor class lies in a given linear } \\
\text { system } \mathbb{P}^{\delta} \subset|L|\end{array}$ \\
\hline$P_{n}(S, \beta)$ & $\begin{array}{l}\text { moduli space of stable pairs }(F, s) \text { on } S \text { with curve class } \\
\beta \text { and holomorphic Euler characteristic } \chi(F)=n\end{array}$ \\
\hline$R_{g, \beta}$ & (30) reduced Gromov-Witten invariant $\in \mathbb{Q}$ \\
\hline $\mathscr{R}_{g, \beta}$ & (34) reduced residue Gromov-Witten invariant $\in \mathbb{Q}(t)$ \\
\hline$P_{n, \beta}^{r e d}$ & (44) reduced stable pair invariant $\in \mathbb{Z}$ \\
\hline $\mathscr{P}_{n, \beta}^{\text {red }}$ & $\begin{array}{l}\text { reduced residue stable pair invariant } \in \mathbb{Z}(t) \text {; see }(35) \text { for } \\
X \text { and }(45) \text { for } S\end{array}$ \\
\hline$r_{g, \beta}$ & (65) reduced residue Gopakumar-Vafa BPS invariants \\
\hline$g=g(C)$ & arithmetic genus of a curve $C$ \\
\hline $\bar{g}:=g(\bar{C})$ & geometric genus $=$ arithmetic genus of normalisation $\bar{C}$ \\
\hline$n_{\delta}(L)$ & $\begin{array}{l}\text { (60) Severi degree counting } \delta \text {-nodal curves in } \\
\delta \text {-dimensional linear subsystems } \mathbb{P}^{\delta} \text { of }|L|\end{array}$ \\
\hline$H_{\beta} \subset H_{\gamma}$ & (74) $\operatorname{Hilb}_{\beta}(S)$ embedded in $\operatorname{Hilb}_{\gamma:=[A]+\beta}(S)$ by $C \mapsto C+A$ \\
\hline
\end{tabular}




\section{Reduced obstruction theories}

\subsection{The algebraic twistor family $\mathcal{S}_{B}$}

Fix a projective surface $S$, and let $\mathfrak{m}$ denote the maximal ideal at the origin $0 \in H^{1}\left(T_{S}\right)$. The first order neighbourhood of the origin

$$
\text { Spec } \mathcal{O}_{H^{1}\left(T_{S}\right)} / \mathfrak{m}^{2}
$$

has a cotangent sheaf whose restriction to the origin is $H^{1}\left(T_{S}\right)^{*}$. Over this Artinian space lies a tautological flat family of surfaces $\mathcal{S}$ with Kodaira-Spencer class the identity in $H^{1}\left(T_{S}\right)^{*} \otimes H^{1}\left(T_{S}\right)$ parametrising the extension

$$
\left.0 \longrightarrow H^{1}\left(T_{S}\right)^{*} \otimes \mathcal{O}_{S} \longrightarrow \Omega_{\mathcal{S}}\right|_{S} \longrightarrow \Omega_{S} \longrightarrow 0 .
$$

Now fix a class $\beta \in H^{1,1}(S) \cap\left(H^{2}(S, \mathbb{Z}) /\right.$ torsion) for which $H^{1}\left(T_{S}\right) \stackrel{\cup \beta}{\longrightarrow} H^{2}\left(\mathcal{O}_{S}\right)$ is a surjection. Picking a splitting we get a $h^{0,2}(S)$-dimensional subspace $V \subset H^{1}\left(T_{S}\right)$ such that

$$
\cup \beta: V \rightarrow H^{2}\left(\mathcal{O}_{S}\right)
$$

is an isomorphism. Restricting the family $\mathcal{S}$ to $V$ gives a flat family

$$
\mathcal{S}_{B} \quad \text { over } B:=\operatorname{Spec} \mathcal{O}_{V} / \mathfrak{m}^{2}=\operatorname{Spec}\left(\mathbb{C} \oplus V^{*}\right)
$$

and an exact sequence of Kähler differentials

$$
\left.\left.0 \longrightarrow \Omega_{B}\right|_{S} \longrightarrow \Omega_{\mathcal{S}_{B}}\right|_{S} \longrightarrow \Omega_{S} \longrightarrow 0 \text {. }
$$

In the first term we have suppressed the pullback map from the base; the result is $V^{*} \otimes \mathcal{O}_{S}$. The extension class of $(7)$ - the Kodaira-Spencer class in $H^{1}\left(T_{S} \otimes V^{*}\right)=\operatorname{Hom}\left(V, H^{1}\left(T_{S}\right)\right)$ of the family $\mathcal{S}_{B}$ - induces an isomorphism

$$
V \underset{\simeq}{\simeq} H^{1}\left(T_{S}\right) \stackrel{\cup \beta}{\longrightarrow} H^{2}\left(\mathcal{O}_{S}\right) .
$$

There is a canonical isomorphism [Blo72, Proposition 3.8]

$$
H_{d R}^{2}\left(\mathcal{S}_{B} / B\right) \cong H^{2}(S, \mathbb{C}) \otimes_{\mathbb{C}} \mathcal{O}_{B}
$$

So corresponding to $\beta \otimes 1$ we get the horizontal lift of $\beta$ :

$$
\beta_{B} \in H_{d R}^{2}\left(\mathcal{S}_{B} / B\right) \text {. }
$$

By projection we obtain a class

$$
\left[\beta_{B}\right]^{0,2} \in H_{d R}^{2}\left(\mathcal{S}_{B} / B\right) / F^{1} H_{d R}^{2}\left(\mathcal{S}_{B} / B\right),
$$

where $F^{1} H_{d R}^{2}\left(\mathcal{S}_{B} / B\right)$ is the part of the Hodge filtration defined by $\Omega_{\mathcal{S}_{B} / B}^{\geqslant 1}$. The scheme theoretic Noether-Lefschetz locus in $B$ is defined to be the zero locus of $\left[\beta_{B}\right]^{0,2}$. The family $\mathcal{S}_{B}$ was constructed precisely to ensure the following.

Lemma 2.1. The Noether-Lefschetz locus in $B$ is just the closed point $0 \in B$. 


\section{Curve COUnting on SuRfaces I: TheOrY}

Proof. Since $\beta$ has type $(1,1)$ on the central fibre $S,\left[\beta_{B}\right]^{0,2}$ certainly vanishes at the origin 0 . Next pick any nonzero tangent vector $v \in V$, thus defining a subscheme $B_{v} \subset B$ by intersecting $B$ with the span $\mathbb{C} v \subset V$ (equivalently, define $B_{v}$ via the ideal $\langle v\rangle^{\perp} \subset V^{*} \subset \mathcal{O}_{B}$ ). By [Blo72, Proposition 4.2], $B_{v}$ lies in the Noether-Lefschetz locus if and only if

$$
\nabla(\beta)=0 \text { in } H^{2}\left(\mathcal{O}_{S}\right) \text {. }
$$

Here $\nabla$ [Blo72, (4.1)] is the map given by cup product with the Kodaira-Spencer class of $\mathcal{S}_{B_{v}}$, that is, the image of $v \in V$ under the first arrow of (8). By (8) then, (9) does not hold, so $B_{v}$ is not in the Noether-Lefschetz locus.

Denote by $j: S \hookrightarrow \mathcal{S}_{B}$ the inclusion of the central fibre, and denote by $\bar{M}_{g, n}\left(\mathcal{S}_{B} / B, \beta_{B}\right) \rightarrow B$ the moduli space of stable maps of connected genus $g$ curves with $n$ marked points to the fibres of $\mathcal{S}_{B} \rightarrow B$.

Proposition 2.2. Recall that we are assuming condition (3). Then the natural morphism of stacks $j_{*}: \bar{M}_{g, n}(S, \beta) \rightarrow \bar{M}_{g, n}\left(\mathcal{S}_{B} / B, \beta_{B}\right)$ is an isomorphism.

Proof. Since $\bar{M}_{g, n}\left(\mathcal{S}_{B} / B, \beta_{B}\right) \times_{B}\{0\} \cong \bar{M}_{g, n}(S, \beta)$ we need only prove the following.

Suppose we have an Artinian scheme $A$ with a morphism to $B$, a proper flat family $C \rightarrow A$ and a $B$-morphism $h: C \rightarrow \mathcal{S}_{B}$ which pulls back to a stable map $h_{0}: C_{0} \rightarrow S$ satisfying $h_{0 *}\left[C_{0}\right]=\beta$. Then we want to show that $A \rightarrow B$ factors through $0 \in B$.

Now define $\mathcal{S}_{A} / A:=\mathcal{S}_{B} \times_{B} A$ with horizontal class

$$
\beta_{A}:=\beta \otimes 1 \in H_{d R}^{2}\left(\mathcal{S}_{A} / A\right) \cong H^{2}(S, \mathbb{C}) \otimes_{\mathbb{C}} \mathcal{O}_{A} .
$$

It is the pullback of $\beta_{B}$ via $A \rightarrow B$. [Blo72, Proposition 5.6] defines the class $h_{*}[C]=\beta_{A} \in$ $F^{1} H_{d R}^{2}\left(\mathcal{S}_{A} / A\right)$. In particular $\left[\beta_{A}\right]^{0,2} \in H_{d R}^{2}\left(\mathcal{S}_{A} / A\right) / F^{1} H_{d R}^{2}\left(\mathcal{S}_{A} / A\right)$ is zero, so that the image of $A$ lies in the zero locus of $\left[\beta_{B}\right]^{0,2}$, which by Lemma 2.1 is scheme-theoretically just the closed point $0 \in B$.

We are also interested in the threefold $X=K_{S}$ that is the total space of the canonical bundle of $S$. For technical reasons it is often convenient to work on its projective completion $\bar{X}:=\mathbb{P}\left(K_{S} \oplus \mathcal{O}_{S}\right)$.

Let $\beta \in H^{2}(S)$ and $\iota: S \hookrightarrow \bar{X}$ be the inclusion of the zero section. Since $\bar{X}$ is a $\mathbb{P}^{1}$-bundle over $S$, there are canonical isomorphisms $H^{1}\left(T_{\bar{X}}\right) \cong H^{1}\left(T_{S}\right)$ and $H^{i, j}(S) \cong H^{i+1, j+1}(\bar{X})$ when $i+j=2$, intertwining $\cup \beta$ with $\cup \iota_{*} \beta$. Associated to the family $\mathcal{S}_{B} \rightarrow B$ (6) we also get families of threefolds $\mathcal{X}_{B} \rightarrow B$ and $\overline{\mathcal{X}}_{B} \rightarrow B$, with natural inclusions from $\mathcal{S}_{B}$ which we also denote by $\iota$. As before we let $j$ denote any of the inclusions of the central fibres $S, X, \bar{X}$ into the families $\mathcal{S}_{B}, \mathcal{X}_{B}, \overline{\mathcal{X}}_{B}$

In addition to the moduli space of stable maps $\bar{M}_{g, n}\left(\bar{X}, \iota_{*} \beta\right)$ to $\bar{X}$, we will also want to use moduli spaces of stable pairs [PT09] on $S$ and $\bar{X}$.

Denote by $P_{n}\left(\bar{X}, \iota_{*} \beta\right)$ the (fine) moduli space of stable pairs $(F, s)$ on $\bar{X}$ with $\operatorname{ch}_{2}(F)=\iota_{*} \beta$ and holomorphic Euler characteristic $\chi(F)=n$. Similarly, $P_{n}\left(\overline{\mathcal{X}}_{B} / B, \iota_{*} \beta_{B}\right)$ is the relative moduli 
space of stable pairs on the fibres of $\overline{\mathcal{X}}_{B} \rightarrow B$. We can repeat these definitions for $S$ and $\mathcal{S}_{B} / B$ for stable pairs $(F, s)$ with $c_{1}(F)=\beta$ and $\chi(F)=n$.

Proposition 2.3. Assuming condition (3), the natural morphisms

(i) $j_{*}: \bar{M}_{g, n}(S, \beta) \rightarrow \bar{M}_{g, n}\left(\mathcal{S}_{B} / B, \beta_{B}\right)$,

(ii) $j_{*}: \bar{M}_{g, n}\left(\bar{X}, \iota_{*} \beta\right) \rightarrow \bar{M}_{g, n}\left(\overline{\mathcal{X}}_{B} / B, \iota_{*} \beta_{B}\right)$,

(iii) $j_{*}: P_{n}(S, \beta) \rightarrow P_{n}\left(\mathcal{S}_{B} / B, \beta_{B}\right)$ and

(iv) $j_{*}: P_{n}\left(\bar{X}, \iota_{*} \beta\right) \rightarrow P_{n}\left(\overline{\mathcal{X}}_{B} / B, \iota_{*} \beta_{B}\right)$

are isomorphisms.

Proof. The first is Proposition 2.2. The same proof, with $h_{*}[C]$ replaced by $c_{1}(F)$, gives statement (iii).

For statements (ii) and (iv), the proof is the same once we replace $h_{*}[C]$ by $c h_{2}(F)$ and $H_{d R}^{2}\left(\mathcal{S}_{B} / B\right) / F^{1} H_{d R}^{2}\left(\mathcal{S}_{B} / B\right)$ by $H_{d R}^{4}\left(\overline{\mathcal{X}}_{B} / B\right) / F^{2} H_{d R}^{4}\left(\overline{\mathcal{X}}_{B} / B\right)$.

\subsection{Stable maps}

In this section we abbreviate the notation $\bar{M}_{g, n}(S, \beta)$ for the moduli space of stable maps with $n$ marked points to $\bar{M}$. When $n=0$ and $f: C \hookrightarrow S$ is an embedded curve with normal bundle $N_{C}$, the natural deformation-obstruction theory for stable maps is

$$
E^{\bullet}:=R \Gamma\left(N_{C}\right)^{\vee}
$$

at the point $f \in \bar{M}$. This naturally extends to a two-term complex over $\bar{M}$ with a morphism to the truncated cotangent complex $\mathbb{L}_{\bar{M}}:=\tau^{\geqslant-1} L_{\bar{M}}^{\bullet}$ of $\bar{M}$. The semi-regularity map $h^{1}\left(\left(E^{\bullet}\right)^{\vee}\right)=$ $H^{1}\left(N_{C}\right) \rightarrow H^{2}\left(\mathcal{O}_{S}\right)$ of (1) is Serre dual to the composition

$$
H^{0}\left(K_{S}\right) \longrightarrow H^{0}\left(f^{*} \Omega_{S} \otimes f^{*} \Omega_{S}\right) \longrightarrow H^{0}\left(N_{C}^{*} \otimes \omega_{C}\right),
$$

where all of the maps are the obvious ones.

For a general stable map $f:\left(C, x_{1} \ldots, x_{n}\right) \rightarrow S$, we replace $N_{C}^{*}$ by the complex $\left\{f^{*} \Omega_{S} \rightarrow\right.$ $\left.\Omega_{C}\left(x_{1}+\ldots+x_{n}\right)\right\}$. Here $f^{*} \Omega_{S}$ is placed in degree 0 . When $C$ is embedded and $n=0$, the arrow is surjective so the complex is indeed quasi-isomorphic to its kernel $N_{C}^{*}$. More globally, denote the universal curve by

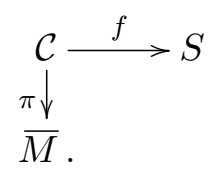

Then the perfect obstruction theory of Gromov-Witten theory is [Beh97, BeF97]

$$
E^{\bullet}:=\left(R \pi_{*} R \mathscr{H} \text { om }\left(\left\{f^{*} \Omega_{S} \rightarrow \Omega_{\mathcal{C} / \bar{M}}^{\log }\right\}, \mathcal{O}_{\mathcal{C}}\right)\right)^{\vee} \longrightarrow \mathbb{L}_{\bar{M}},
$$

where

$$
\Omega_{\mathcal{C} / \bar{M}}^{\log }:=\Omega_{\mathcal{C} / \bar{M}}\left(x_{1}+\cdots+x_{n}\right)
$$




\section{Curve COUNTING ON SURFACES I: THEORY}

and $x_{1}, \ldots, x_{n}$ are the sections of the universal curve defining the marked points. Since marked points are always smooth points of the curve, (13) is the sheaf of logarithmic one-forms. Letting $\omega_{\mathcal{C} / \bar{M}}$ be the relative dualising line bundle, consider the composition of the following standard maps

$$
\begin{aligned}
f^{*} K_{S} \rightarrow f^{*} \Omega_{S} \otimes f^{*} \Omega_{S} & \\
\downarrow & \left\{f^{*} \Omega_{S} \longrightarrow \Omega_{\mathcal{C} / \bar{M}}\right\} \otimes \Omega_{\mathcal{C} / \bar{M}} \rightarrow\left\{f^{*} \Omega_{S} \rightarrow \Omega_{\mathcal{C} / \bar{M}}^{\log }\right\} \otimes \omega_{\mathcal{C} / \bar{M}} .
\end{aligned}
$$

The composition $f^{*} K_{S} \rightarrow \Omega_{\mathcal{C} / \bar{M}} \otimes \Omega_{\mathcal{C} / \bar{M}}$ of the first arrow and the dotted arrow is zero since it factors through $\Lambda^{2} \Omega_{\mathcal{C} / \bar{M}}=0$. Therefore (14) defines a map of complexes

$$
f^{*} K_{S} \longrightarrow\left\{f^{*} \Omega_{S} \rightarrow \Omega_{\mathcal{C} / \bar{M}}^{\log }\right\} \otimes \omega_{\mathcal{C} / \bar{M}}
$$

Composing with $H^{0}\left(K_{S}\right) \rightarrow H^{0}\left(f^{*} K_{S}\right)$ and applying $R \pi_{*}$ we get the (dual) semi-regularity map

$$
H^{0}\left(K_{S}\right) \otimes \mathcal{O}_{\bar{M}} \longrightarrow E^{\bullet}[-1]
$$

which reduces to (10) when $f$ is an embedding and there are no marked points. Dualising and taking $h^{1}$ gives

$$
\mathrm{ob} \longrightarrow H^{2}\left(\mathcal{O}_{S}\right) \otimes \mathcal{O}_{\bar{M}}
$$

where ob $:=h^{1}\left(\left(E^{\bullet}\right)^{\vee}\right)$ is the obstruction sheaf on $\bar{M}$. We will see that this is a surjection in the proof of Theorem 2.4.

We define the reduced obstruction theory of $\bar{M}$ to be the cone on the map (15):

$$
E_{\text {red }}^{\bullet}:=\operatorname{Cone}\left(H^{0}\left(K_{S}\right) \otimes \mathcal{O}_{\bar{M}}[1] \rightarrow E^{\bullet}\right) .
$$

Its name is justified by the next result.

THEOREm 2.4. Suppose that $\beta \in H^{2}(S, \mathbb{Z})$ is a $(1,1)$ class such that $H^{1}\left(T_{S}\right) \stackrel{\cup \beta}{\longrightarrow} H^{2}\left(\mathcal{O}_{S}\right)$ is surjective. Then there is a perfect obstruction theory ${ }^{4} E_{\text {red }}^{\bullet} \rightarrow \mathbb{L}_{\bar{M}}$ for $\bar{M}_{g, n}(S, \beta)$ with virtual dimension

$$
v:=g-1+\int_{\beta} c_{1}(S)+n+h^{0,2}(S) .
$$

Proof. By [Beh97, BeF97] the relative obstruction theory for $\bar{M}_{g, n}\left(\mathcal{S}_{B} / B, \beta_{B}\right)$ is

$$
E_{r e l}^{\bullet}:=\left(R \pi_{*} R \mathscr{H} o m\left(\left\{f^{*} \Omega_{\mathcal{S}_{B} / B} \rightarrow \Omega_{\mathcal{C} / \bar{M}}^{\log }\right\}, \mathcal{O}_{\mathcal{C}}\right)\right)^{\vee} \longrightarrow \mathbb{L}_{\bar{M}_{g, n}\left(\mathcal{S}_{B} / B, \beta_{B}\right) / B},
$$

\footnotetext{
${ }^{4}$ While $E_{r e d}^{\bullet}$ is canonical, we are currently unable to prove that the map $E_{r e d}^{\bullet} \rightarrow \mathbb{L}_{\bar{M}}$ is independent of the choice (5) made to define the algebraic twistor family $\mathcal{S}_{B}$. But the induced virtual cycle is canonical, since it only depends on the K-theory class of $E_{r e d}^{\bullet}$ [Pid91, Sie04].
} 
where the maps are those of the universal diagram

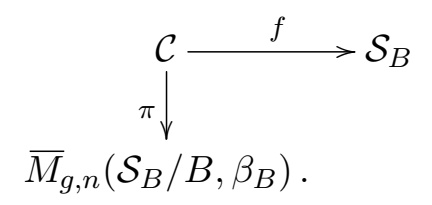

By Proposition $2.2, \bar{M}_{g, n}\left(\mathcal{S}_{B} / B, \beta_{B}\right) \cong \bar{M}$ and the above diagram factors through the diagram (11) for $S$ instead of $\mathcal{S}_{B}$. Therefore in fact $E_{r e l}^{\bullet}$ is just $E^{\bullet}$ (12), giving a perfect relative obstruction theory

$$
E^{\bullet} \longrightarrow \mathbb{L}_{\bar{M} / B}
$$

Even though $B$ is not smooth, its simple form - and the fact that $\bar{M}$ is supported over the reduced point $0 \in B$ - means that there is an exact triangle

$$
\left.\left.\Omega_{B}\right|_{\bar{M}} \longrightarrow \mathbb{L}_{\bar{M}} \longrightarrow \mathbb{L}_{\bar{M} / B} \longrightarrow \Omega_{B}\right|_{\bar{M}}[1]
$$

where again we have suppressed the pullback map from $B$. Explicitly, embed $\bar{M}$ into an ambient stack $\mathcal{A} \rightarrow B$ which is smooth over $B$, and let $I$ denote the ideal of $\bar{M} \subset \mathcal{A}$. Then the triangle comes from the horizontal exact sequence of vertical complexes

$$
\begin{gathered}
I / I^{2}=I / I^{2} \\
\downarrow \\
\downarrow \\
\downarrow
\end{gathered}
$$

where the exactness of the bottom row follows from the smoothness of $\mathcal{A} \rightarrow B$. From (19) and (20) we get a map $\left.E^{\bullet}[-1] \rightarrow \Omega_{B}\right|_{\bar{M}}$ whose cone we define to be $F^{\bullet}$ :

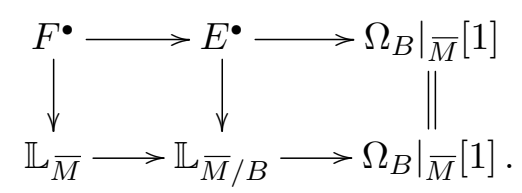

To show that $F^{\bullet}=\operatorname{Cone}\left(\left.E^{\bullet}[-1] \rightarrow \Omega_{B}\right|_{\bar{M}}\right)$ is quasi-isomorphic to $E_{r e d}^{\bullet}=\operatorname{Cone}\left(H^{0}\left(K_{S}\right) \otimes\right.$ $\left.\mathcal{O}_{\bar{M}}[1] \rightarrow E^{\bullet}\right)$ it is sufficient to show that the composition

$$
\left.H^{0}\left(K_{S}\right) \otimes \mathcal{O}_{\bar{M}} \longrightarrow E^{\bullet}[-1] \longrightarrow \Omega_{B}\right|_{\bar{M}}
$$

is an isomorphism. The dual of this isomorphism says that moving in any direction in $B$, our curve is obstructed at first order since its cohomology class acquires a nonzero $(0,2)$ part $[\beta]^{0,2}$. It also proves the surjectivity claimed in (16).

Applying $h^{0}$ we have

$$
\left.H^{0}\left(K_{S}\right) \otimes \mathcal{O}_{\bar{M}} \longrightarrow R^{0} \pi_{*}\left(\left\{f^{*} \Omega_{S} \rightarrow \Omega_{\mathcal{C} / \bar{M}}^{\log }\right\} \otimes \omega_{\mathcal{C} / \bar{M}}\right) \longrightarrow \Omega_{B}\right|_{\bar{M}}
$$

Recalling the definition of the dual semi-regularity map (15) via the diagram (14) we see this 


\section{CURVE COUNTING ON SURFACES I: THEORY}

factors through

$$
\left.H^{0}\left(K_{S}\right) \otimes \mathcal{O}_{\bar{M}} \longrightarrow R^{0} \pi_{*}\left(f^{*} \Omega_{S} \otimes \omega_{\mathcal{C} / \bar{M}}\right) \longrightarrow \Omega_{B}\right|_{\bar{M}},
$$

where the first arrow is the obvious map pulling back two-forms to $C$. By Lemma 2.5 below the second arrow - induced by the Kodaira-Spencer map of $\bar{M} / B$ in the bottom row of $(21)$ - is the same as the one

$$
\left.R^{0} \pi_{*}\left(f^{*} \Omega_{S} \otimes \omega_{\mathcal{C} / \bar{M}}\right) \longrightarrow R^{1} \pi_{*}\left(\left.\Omega_{B}\right|_{\bar{M}} \otimes \omega_{\mathcal{C} / \bar{M}}\right) \cong \Omega_{B}\right|_{\bar{M}}
$$

induced by the Kodaira-Spencer map $\left.\Omega_{S} \rightarrow \Omega_{B}\right|_{\bar{M}}[1](7)$ of $\mathcal{S}_{B} / B$. The composition is therefore the dual of (8) pulled back to $\bar{M}$, which is indeed an isomorphism.

By the long exact sequences in cohomology of diagram (21), and the fact that $E^{\bullet}$ is a perfect relative obstruction theory, we see that $F^{\bullet}$ has cohomology only in degrees $-1,0$ and that $F^{\bullet} \rightarrow \mathbb{L}_{\bar{M}}$ is an isomorphism on $h^{0}$ and a surjection on $h^{-1}$. And since $E^{\bullet}$ is quasi-isomorphic to a two-term complex of vector bundles $\left\{E^{-1} \rightarrow E^{0}\right\}$, so is $F^{\bullet}$ locally:

$$
F^{\bullet} \simeq\left\{\left.E^{-1} \rightarrow E^{0} \oplus \Omega_{B}\right|_{\bar{M}}\right\} .
$$

(We work locally to obtain the map $E^{-1} \rightarrow \Omega_{B}$ from the fact that $E^{-1}$ is free, thus defining a projective module.) Working globally one can do the same thing by resolving by sufficiently negative locally free sheaves, etc. Thus $F^{\bullet} \rightarrow \mathbb{L}_{\bar{M}}$ is an absolute obstruction theory for $\bar{M}$.

LEMma 2.5. The second arrow in (23) is the one induced by the Kodaira-Spencer map $\Omega_{S} \rightarrow$ $\left.\Omega_{B}\right|_{\bar{M}}[1](7)$ of $\mathcal{S}_{B} / B$.

Proof. Let $\overline{\mathcal{M}}:=\overline{\mathcal{M}}_{g, n}$ denote the stack of prestable curves with $n$ marked points, with universal curve $\mathcal{C} \rightarrow \overline{\mathcal{M}}$.

In passing from (22) to (23) we have passed from the obstruction theory $E^{\bullet} \rightarrow \mathbb{L}_{\bar{M} / B}$ (composed with the Kodaira-Spencer map $\left.\mathbb{L}_{\bar{M} / B} \rightarrow \Omega_{B}\right|_{\bar{M}}[1]$ of $\left.\bar{M}\right)$ to the relative obstruction theory $\mathcal{E}^{\bullet} \rightarrow \mathbb{L}_{\bar{M} / \overline{\mathcal{M}} \times B}$ (composed with the Kodaira-Spencer map $\mathbb{L}_{\bar{M} / \overline{\mathcal{M}} \times B} \rightarrow \Omega_{B}[1]$ of $\bar{M} / \overline{\mathcal{M}}$ ). In other words the second arrow in (23) is the composition

$$
\left.h^{-1}\left(\mathcal{E}^{\bullet}\right) \longrightarrow h^{-1}\left(\mathbb{L}_{\bar{M} / \overline{\mathcal{M}} \times B}\right) \longrightarrow \Omega_{B}\right|_{\bar{M}} .
$$

We recall the relative obstruction theory of $\bar{M} / \overline{\mathcal{M}} \times B$ [Beh97, BeF97], using the maps

$$
\begin{gathered}
\mathcal{C} \times \overline{\mathcal{M}} \bar{M} \stackrel{f}{\longrightarrow} \mathcal{S}_{B} \\
\underset{\downarrow}{\downarrow} \pi \\
\end{gathered}
$$

In fact, it is simpler to describe the dual of the perfect obstruction theory $\mathcal{E}^{\bullet} \rightarrow \mathbb{L} \mathbb{L}_{\bar{M} / \overline{\mathcal{M}} \times B}$; it is the composition $\mathbb{L} \frac{\vee}{\bar{M} / \overline{\mathcal{M}} \times B} \rightarrow R \pi_{*}\left(f^{*} T_{\mathcal{S}_{B} / B}\right)$ of the top row of the diagram of vertical exact 
triangles

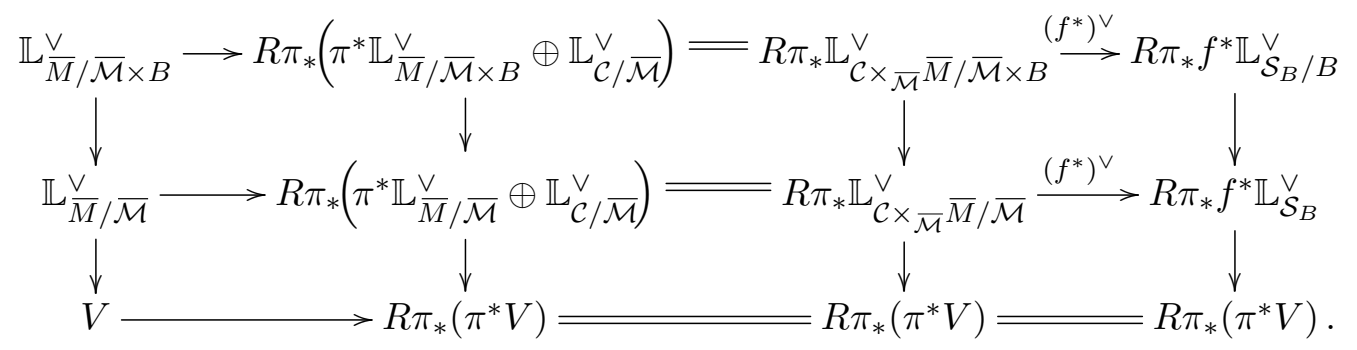

Here $\mathbb{L}_{\mathcal{S}_{B} / B}^{\vee}$ is just the tangent bundle $T_{\mathcal{S}_{B} / B}$ since $\mathcal{S}_{B} \rightarrow B$ is smooth, $V$ is the splitting of (5), and we recall that $\left.\Omega_{B}\right|_{\bar{M}} \cong V^{*}$.

Use the natural exact triangle of cohomologies

$$
V \longrightarrow R \pi_{*}\left(\pi^{*} V\right) \longrightarrow R^{1} \pi_{*}\left(\pi^{*} V\right)[-1]
$$

to remove $R^{1} \pi_{*}\left(\pi^{*} V\right)[-1]$ from the lower and the middle terms in the right-hand column. In the latter case we call the resulting cone $\left(\mathcal{F}^{\bullet}\right)^{\vee}$. Now compose (26) horizontally and dualise, to give a commutative diagram involving just the first and last columns:

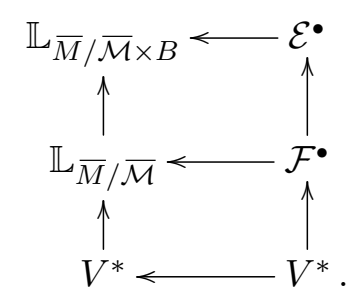

Rearranging gives

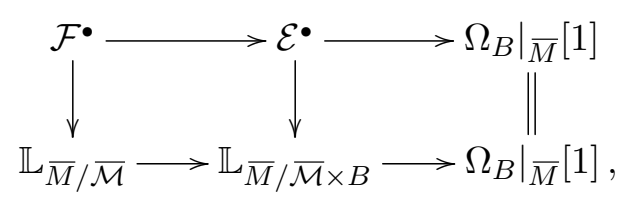

just as in (21), but for $\mathbb{L}_{\bar{M} / \overline{\mathcal{M}}}$ instead of $\mathbb{L}_{\bar{M}}$. But (26) commutes, so the above diagram does too. The top right-hand map is the one we are after (after taking $h^{-1}$ ), and from the last column of (26) we see that it is indeed the one induced by the Kodaira-Spencer map of $\mathcal{S}_{B}$ over $B$.

If we work on $\bar{X}=\mathbb{P}\left(K_{S} \oplus \mathcal{O}_{S}\right)$ instead of $S$ we get a similar semi-regularity map by replacing $K_{S}$ and $\Omega_{S}$ in (14) by $\Omega_{\bar{X}}^{2}$ and $\Omega_{\bar{X}}$, respectively. Thus we remove $H^{1,3}(\bar{X}) \cong H^{0,2}(S)$ from the obstruction sheaf, and by very similar working obtain the following.

Theorem 2.6. Suppose that $\beta \in H^{2}(S, \mathbb{Z})$ is a $(1,1)$ class inducing a surjection $\cup \beta: H^{1}\left(T_{S}\right) \rightarrow$ $H^{2}\left(\mathcal{O}_{S}\right)$. Then there is a perfect obstruction theory $E_{\text {red }}^{\bullet} \rightarrow \mathbb{L}_{\bar{M}}$ for $\bar{M}_{g, n}\left(\bar{X}, \iota_{*} \beta\right)$ with virtual dimension $h^{1,3}(\bar{X})+n=h^{0,2}(S)+n$. 


\section{Curve COUNTING ON SURFACES I: THEORY}

\subsection{Stable pairs}

We sketch how the arguments of the last section are modified to prove the same result for stable pairs. In Appendix 5.2 we use a different method to get a better result using only condition (2) in place of $(3)$.

As before we let $\bar{X}=\mathbb{P}\left(K_{S} \oplus \mathcal{O}_{S}\right)$ be the projective completion of the canonical bundle of $S$. Let $0 \neq \beta \in H_{2}(S, \mathbb{Z})$ be of type $(1,1)$ and denote the inclusion of the zero section by $\iota: S \hookrightarrow \bar{X}$.

Let $P:=P_{n}\left(\bar{X}, \iota_{*} \beta\right)$ be the moduli space of stable pairs on $\bar{X}$ with universal object $\mathbb{I}^{\bullet}=$ $\left\{\mathcal{O}_{\bar{X} \times P} \longrightarrow \mathbb{F}\right\}$ over $\bar{X} \times P$. Using the projections

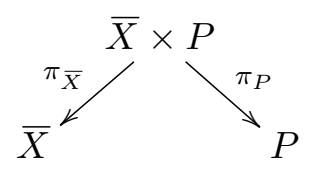

and the relative dualising sheaf $\omega_{\pi_{P}}=\pi_{\bar{X}}^{*} \omega_{\bar{X}}$, the perfect obstruction theory for stable pair theory of $\bar{X}$ is [PT09, Theorem 2.14]

$$
E^{\bullet}:=R \pi_{P *}\left(R \mathscr{H} o m\left(\mathbb{I}^{\bullet}, \mathbb{I}^{\bullet}\right)_{0} \otimes \omega_{\pi_{P}}\right)[2] \longrightarrow \mathbb{L}_{P} .
$$

Here $(\cdot)_{0}$ denotes the trace-free part, and the virtual dimension is $\int_{\iota_{*} \beta} c_{1}(\bar{X})=0$. This gives an obstruction sheaf

$$
\mathrm{Ob}:=h^{1}\left(\left(E^{\bullet}\right)^{\vee}\right)=\mathscr{E} x t_{\pi_{P}}^{2}\left(\mathbb{I}^{\bullet}, \mathbb{I}^{\bullet}\right)_{0},
$$

where $\mathscr{E} x t_{\pi_{P}}^{i}$ denotes the $i$ th cohomology sheaf of $R \pi_{P *} R \mathscr{H} o m$. Cupping with the Atiyah class $A\left(\mathbb{I}^{\bullet}\right) \in \operatorname{Ext}^{1}\left(\mathbb{I}^{\bullet}, \mathbb{I}^{\bullet} \otimes \mathbb{L}_{\bar{X} \times P}\right)$ and taking the trace defines a semi-regularity map $\left(E^{\bullet}\right)^{\vee} \rightarrow$ $H^{1,3}(\bar{X}) \otimes \mathcal{O}_{P}[-1]$ by the composition

$$
\begin{aligned}
\mathscr{E} x t_{\pi_{P}}^{2}\left(\mathbb{I}^{\bullet}, \mathbb{I}^{\bullet}\right)_{0} \subset & \mathscr{E} x t_{\pi_{P}}^{2}\left(\mathbb{I}^{\bullet}, \mathbb{I}^{\bullet}\right) \stackrel{\cup A\left(\mathbb{I}^{\bullet}\right)}{\longrightarrow} \mathscr{E} x t_{\pi_{P}}^{3}\left(\mathbb{I}^{\bullet}, \mathbb{I}^{\bullet} \otimes \mathbb{L}_{\bar{X} \times P}\right) \longrightarrow \\
& \mathscr{E} x t_{\pi_{P}}^{3}\left(\mathbb{I}^{\bullet}, \mathbb{I}^{\bullet} \otimes \pi_{\bar{X}}^{*} \Omega_{\bar{X}}\right) \stackrel{\operatorname{tr}}{\longrightarrow} R^{3} \pi_{P *} \pi_{\bar{X}}^{*} \Omega_{\bar{X}} \cong H^{1,3}(\bar{X}) \otimes \mathcal{O}_{P} .
\end{aligned}
$$

We will see in the proof of Theorem 2.7 that $(28)$ is a surjection when $(S, \beta)$ satisfy condition (3). This also follows the obvious generalisation of [MPT10, Proposition 11] to all surfaces.

Dualising the composition

$$
\left(E^{\bullet}\right)^{\vee} \longrightarrow h^{1}\left(\left(E^{\bullet}\right)^{\vee}\right)[-1] \longrightarrow H^{1,3}(\bar{X}) \otimes \mathcal{O}_{P}[-1]
$$

gives a map

$$
H^{2,0}(\bar{X}) \otimes \mathcal{O}_{P}[1] \longrightarrow E^{\bullet}
$$

let $E_{r e d}^{\bullet}$ be its cone.

Theorem 2.7. Assume that $\cup \beta: H^{1}\left(T_{S}\right) \rightarrow H^{2}\left(\mathcal{O}_{S}\right)$ is surjective. Then there exists a perfect obstruction theory $E_{\text {red }}^{\bullet} \rightarrow \mathbb{L}_{P}$ for $P_{n}\left(\bar{X}, \iota_{*} \beta\right)$ of virtual dimension $h^{1,3}(\bar{X})=h^{0,2}(S)$.

Proof. Associated with the algebraic twistor family $\mathcal{S}_{B} \rightarrow B$ we get its family of projectively completed canonical bundles $\overline{\mathcal{X}}_{B} \rightarrow B$. By Proposition 2.3 the family $P:=P_{n}\left(\overline{\mathcal{X}}_{B} / B, \iota_{*} \beta_{B}\right) \rightarrow B$ 


\section{Kool, R. P. Thomas}

of moduli spaces of stable pairs on the fibres is isomorphic to the space $P_{n}(\bar{X}, \beta)$ of stable pairs on $\bar{X}$.

In [MPT10, Section 3], a relative perfect obstruction theory $E_{r e l}^{\bullet}$ is constructed

$$
E_{\text {rel }}^{\bullet}:=R \pi_{P *}\left(R \mathscr{H} O m\left(\mathbb{I}^{\bullet}, \mathbb{I}^{\bullet}\right)_{0} \otimes \omega_{P \times_{B} \overline{\mathcal{X}}_{B} / P}\right)[2] \longrightarrow \mathbb{L}_{P / B}^{\bullet}
$$

Here $\overline{\mathcal{X}}_{B} \times{ }_{B} P$ carries the universal object $\mathbb{I} \bullet$ and has a projection $\pi_{P}$ to $P$ with relative dualising sheaf $\omega_{\pi_{P}}=\pi_{P}^{*} \omega_{\overline{\mathcal{X}}_{B} / B}$. As before, this can be made into a perfect absolute obstruction theory $F^{\bullet}$ by the diagram

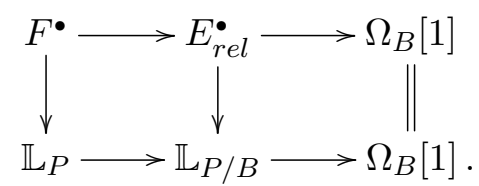

Since by Proposition 2.3 the stable pairs of $P$ all lie scheme-theoretically on the central fibre $\bar{X}$, we see as before that in fact $E_{r e l}^{\bullet}$ is just the usual complex $E^{\bullet}$ of stable pair theory on $\bar{X}$. But $E^{\bullet}$ has virtual dimension 0 , so $F^{\bullet}$ has virtual dimension $h^{2,0}(S)$. Therefore to prove the theorem we are left with showing that the composition

$$
F^{\bullet} \longrightarrow E^{\bullet} \longrightarrow E_{\text {red }}^{\bullet}
$$

is an isomorphism. It is sufficient to show that the composition

$$
\left.H^{0}\left(\Omega_{\bar{X}}^{2}\right) \otimes \mathcal{O}_{P} \longrightarrow E^{\bullet}[-1] \longrightarrow \Omega_{B}\right|_{\bar{M}}
$$

is an isomorphism. By the Nakayama lemma we may do so at a point $(F, s) \in P$. After dualising we get the map $V \rightarrow H^{1,3}(\bar{X})$ given by the composition

$$
\begin{aligned}
V \subset H^{1}\left(T_{S}\right)=H^{1}\left(T_{\bar{X}}\right) & \stackrel{\cup A\left(I^{\bullet}\right)}{\longrightarrow} \operatorname{Ext}^{2}\left(I^{\bullet}, I^{\bullet}\right)_{0} \subset \operatorname{Ext}^{2}\left(I^{\bullet}, I^{\bullet}\right) \\
& \stackrel{\cup A\left(I^{\bullet}\right)}{\longrightarrow} \operatorname{Ext}^{3}\left(I^{\bullet}, I^{\bullet} \otimes \Omega_{\bar{X}}\right) \stackrel{\operatorname{tr}}{\longrightarrow} H^{1,3}(\bar{X}) .
\end{aligned}
$$

This uses the stable pairs analogue of Lemma 2.5 (also proved in [MPT10, Proposition 13]) to deduce that the composition of $E^{\bullet} \rightarrow \mathbb{L}_{P / B}$ and the Kodaira-Spencer map $\mathbb{L}_{P / B} \rightarrow \Omega_{B}[1]$ for $P$ coincides with the cup product of the Atiyah class and the Kodaira-Spencer class for $\bar{X}$.

In the proof of [MPT10, Proposition 11], it is observed that the above composition $H^{1}\left(T_{\bar{X}}\right) \rightarrow$ $H^{1,3}(\bar{X})$ is equal to $\cup\left(-2 \iota_{*} \beta\right)$. Thus on restriction to $V \subset H^{1}\left(T_{S}\right)$ it gives -2 times the isomorphism $\cup \beta$ of (8). 


\section{Curve COUNTING ON SURFACES I: THEORY}

\section{Invariants}

\subsection{Reduced Gromov-Witten invariants}

The reduced obstruction theory of Theorem 2.4 gives, by [BeF97], a virtual fundamental class which we call the reduced class:

$$
\left[\bar{M}_{g, n}(S, \beta)\right]^{r e d} \in H_{2 v}\left(\bar{M}_{g, n}(S, \beta)\right), \quad v=g-1+\int_{\beta} c_{1}(S)+n+h^{0,2}(S) .
$$

Integrating insertion cohomology classes over this gives the reduced Gromov-Witten invariants of $S$. Namely, if $\sigma_{i} \in H^{*}(S, \mathbb{Z})$ are cohomology classes, then

$$
R_{g, \beta}\left(S, \sigma_{1} \ldots \sigma_{n}\right):=\int_{\left[\bar{M}_{g, n}(S, \beta)\right]^{r e d}} \prod_{i=1}^{n} \operatorname{ev}_{i}^{*}\left(\sigma_{i}\right) \in \mathbb{Q} .
$$

Here $\mathrm{ev}_{i}$ is the evaluation map from the $i$ th marked point of the universal curve to $S$. So for a surface in the Noether-Lefschetz locus for $\beta$, the invariants give a virtual count of the curves in homology class $\beta$ which intersect $P D\left(\sigma_{i}\right)$. Notice that the $\sigma_{i}$ can be repeated, so for instance $R_{g, \beta}\left(S, \sigma_{1}^{2} \sigma_{2}\right)$ denotes $R_{g, \beta}\left(S, \sigma_{1} \sigma_{1} \sigma_{2}\right)$.

Remark 3.1. Deformation invariance. By standard theory (Section 7 of [BeF97]), the $R_{g, \beta}\left(\sigma_{1} \ldots \sigma_{n}\right)$ are invariant under deformations of $S$ within the Noether-Lefschetz locus. The usual arguments apply: given a smooth curve $Z$ mapping to the Noether-Lefschetz locus for $\beta$, we can make all of the constructions of the previous sections relative to $Z$ in the family over $Z$. (We do not even need to change notation; we can work with affine $Z$ and just let our ground ring be $\mathcal{O}_{Z}$ instead of $\mathbb{C}$.) The resulting obstruction theory is relative to $Z$, and restricts to the absolute obstruction theory of the previous section over any point of $Z$. As a result the relative virtual cycle on the relative moduli space over $Z$ pulls back, via the usual Gysin maps, to the virtual cycle on any fibre [BeF97, Proposition 7.2]. The cohomology classes $\operatorname{ev}_{i}^{*}\left(\sigma^{i}\right)$ are defined on the relative moduli space, so by conservation of number [Ful98, Theorem 10.2], their integrals over a fibre of the virtual cycle is independent of the fibre. The same applies to the other invariants we define below.

In the usual way we can also define the same invariants (30) without using marked points. Instead we use the universal map $f: \mathcal{C} \rightarrow S$ from the universal curve $\pi: \mathcal{C} \rightarrow \bar{M}_{g}(S, \beta):=$ $\bar{M}_{g, 0}(S, \beta)$. Then we claim that

$$
R_{g, \beta}\left(S, \sigma_{1} \ldots \sigma_{n}\right)=\int_{\left.\left[\bar{M}_{g}(S, \beta)\right]\right]^{r e d}} \prod_{i=1}^{n} \pi_{*} f^{*}\left(\sigma_{i}\right) .
$$


In fact, we can remove one marked point at a time using the diagram

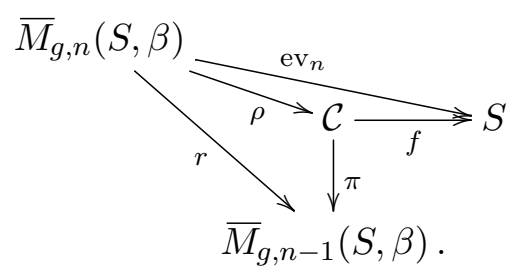

The map $r$ forgets the $n$th marked point and stabilises the resulting curve and map, while $\rho$ maps the $n$th point to its image in the contracted curve. Since $\rho$ is birational, we find that

$$
r_{*} \operatorname{ev}_{n}^{*}(\sigma)=r_{*} \rho^{*} f^{*}(\sigma)=\pi_{*} f^{*}(\sigma) .
$$

Iterating we can push all the way down from $\bar{M}_{g, n}$ to $\bar{M}_{g}$. The compatibility of the ordinary obstruction theories of $\bar{M}_{g, n}$ and $\bar{M}_{g, n-1}$ is [Beh97, Axiom IV]. For the reduced theories the same argument applies because they are defined by the semi-regularity map (15) which is compatible with $r$ : its construction (14) does not even see the marked points. The equality of (30) and (31) follows.

Since $\bar{M}_{g, n}\left(X, \iota_{*} \beta\right) \subset \bar{M}_{g, n}\left(\bar{X}, \iota_{*} \beta\right)$ and $P_{n}\left(X, \iota_{*} \beta\right) \subset P_{n}\left(\bar{X}, \iota_{*} \beta\right)$ are open immersions, they inherit the reduced obstruction theories of Theorems 2.6 and 2.7 by restriction. But they are noncompact, so to define invariants we have to use residues and the virtual localisation formula. The group $T:=\mathbb{C}^{*}$ acts with weight one on the fibres of $X=K_{S}$ with fixed locus $S$. Therefore it acts on the moduli spaces $\bar{M}_{g, n}\left(X, \iota_{*} \beta\right)$ and $P_{n}\left(X, \iota_{*} \beta\right)$. Its fixed loci are related to the curves in the zero-section $S$. For stable maps we get precisely $\bar{M}_{g, n}(S, \beta)$.

Proposition 3.2. The inclusion $\bar{M}_{S} \hookrightarrow \bar{M}_{X}^{T}$ is an isomorphism of stacks. Moreover $E_{X, r e d}^{\bullet}$ is naturally $T$-equivariant and its restriction to $\bar{M}_{S}$ has fixed and moving parts

$$
\begin{aligned}
&\left(\left.E_{X, r e d}^{\bullet}\right|_{\bar{M}_{X}^{T}}\right)^{f i x} \cong E_{S, \text { red }}^{\bullet}, \\
&\left(\left.E_{X, \text { red }}^{\bullet}\right|_{\bar{M}_{X}^{T}}\right)^{\text {mov }} \cong\left(R \pi_{*} f^{*} K_{S} \otimes \mathfrak{t}\right)^{\vee},
\end{aligned}
$$

where $\mathfrak{t}$ is the irreducible representation of weight one.

Proof. The isomorphism

$$
\left.T_{X}\right|_{S} \cong T_{S} \oplus K_{S}
$$

induces an isomorphism on $\bar{M}_{S}$,

$$
\left.E_{X}^{\bullet}\right|_{\bar{M}_{S}} \cong E_{S}^{\bullet} \oplus\left(R \pi_{*}\left(f^{*} K_{S}\right)\right)^{\vee} .
$$

The first summand carries the trivial $T$-action, the second carries the weight $(-1)$ action induced from the action on the fibres of $K_{S}$.

We want to show that the inclusion $\bar{M}_{S} \hookrightarrow \bar{M}_{X}^{T}$ is an isomorphism of stacks. It is sufficient to show that it induces an isomorphism on maps from Spec $A_{n}$ to the moduli space, where $A_{n}$ 


\section{Curve COUnting on Surfaces I: THEORY}

is any Artinian $\mathbb{C}$-algebra of length $n$. The $n=0$ case is the obvious fact that $\bar{M}_{S} \hookrightarrow \bar{M}_{X}^{T}$ is a bijection of sets.

Inductively we fix a surjection $A_{n+1} \rightarrow A_{n}$ with ideal $I$, and a map

$$
a: \operatorname{Spec} A_{n} \rightarrow \bar{M}_{S} \hookrightarrow \bar{M}_{X}^{T} \text {. }
$$

We show that any lift to a map Spec $A_{n+1} \rightarrow \bar{M}_{X}^{T}$ factors through $\bar{M}_{S}$. By [BeF97, Theorem 4.5] such a lift exists if and only if the obstruction class in $\operatorname{Ext}^{1}\left(a^{*}\left(\left.E_{X}^{\bullet}\right|_{M_{X}^{T}}\right)^{f i x}, I\right)$ vanishes. (Here we have used the fact that the $T$-fixed part

$$
\left(\left.E_{X}^{\bullet}\right|_{\bar{M}_{X}^{T}}\right)^{f i x}
$$

of $\left.E_{X}^{\bullet}\right|_{M_{X}^{T}}$ provides an obstruction theory for $\bar{M}_{X}^{T}$ [GP99].)

By the isomorphism (33) this is the same as the obstruction in $\operatorname{Ext}^{1}\left(a^{*} E_{S}^{\bullet}, I\right)$ to finding a lift to $\bar{M}_{S}$. So if a lift to $\bar{M}_{X}^{T}$ exists, so does one to $\bar{M}_{S}$. By [BeF97, Theorem 4.5] and (33) the choices in such a lift are also the same

$$
\operatorname{Hom}\left(a^{*}\left(\left.E_{X}^{\bullet}\right|_{M_{X}^{T}}\right)^{f i x}, I\right) \cong \operatorname{Hom}\left(a^{*} E_{S}^{\bullet}, I\right) .
$$

It follows that the lifts that factor through $\bar{M}_{S}$ map isomorphically to the lifts to $\bar{M}_{X}^{T}$, as required.

Finally, by their very constructions, the semi-regularity maps of $\bar{X}, S$ intertwine the isomorphism (33):

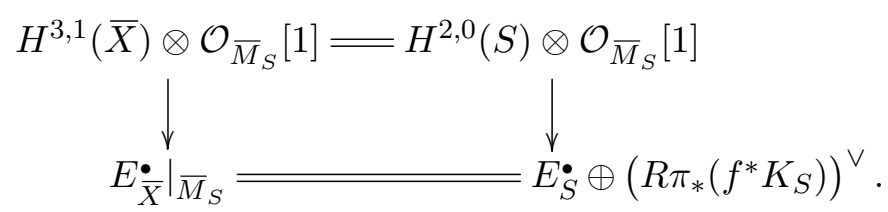

Taking cones gives the isomorphisms

$$
\left.E_{X, \text { red }}^{\bullet}\right|_{\bar{M}_{S}} \cong E_{S, \text { red }}^{\bullet} \oplus\left(R \pi_{*}\left(f^{*} K_{S}\right)\right)^{\vee}
$$

over $\bar{M}_{S} \cong \bar{M}_{X}^{T}$

Therefore we can define reduced Gromov-Witten residue invariants of $X$ using GraberPandharipande's virtual localisation formula [GP99]. That is, via $\left(\left.E_{X, r e d}\right|_{\bar{M}_{X}^{T}}\right)^{f i x}$ we get a perfect obstruction theory for $\bar{M}_{X}^{T}$ and so a virtual cycle $\left[\bar{M}_{X}^{T}\right]^{r e d}$. Then, given equivariant cohomology classes $A_{i} \in H_{T}^{*}\left(\bar{M}_{X}\right)$, we define

$$
\int_{\left[\bar{M}_{X}\right]^{r e d}} \prod_{i} A_{i}:=\int_{\left[\bar{M}_{X}^{T}\right]^{r e d}} \frac{1}{e\left(N^{\text {vir }}\right)} \prod_{i} A_{i} \in \mathbb{Q}(t) .
$$

Here $t=c_{1}(\mathfrak{t})$ is the equivariant parameter - the generator of $H^{*}(B T)=\mathbb{Q}[t]-$ and the virtual normal bundle is defined to be $\left(\left.E_{X, r e d}^{\bullet}\right|_{\bar{M}_{X}^{T}}\right)^{\vee m o v}$. We may express this as a two-term complex 
$E_{0} \rightarrow E_{1}$ of equivariant bundles all of whose weights are nonzero so that the $c_{t o p}\left(E_{i}\right)$ are invertible in the localised equivariant cohomology ring. Here $c_{t o p}$ is the $T$-equivariant top Chern class. Then the virtual equivariant Euler class of $\left(\left.E_{X, r e d}^{\bullet}\right|_{\bar{M}_{X}^{T}} ^{T}\right)^{\vee \text { mov }}$ is defined to be

$$
e\left(N^{v i r}\right):=c_{t o p}\left(E_{0}\right) / c_{t o p}\left(E_{1}\right) \in H_{T}^{*}\left(\bar{M}_{X}^{T}\right) \otimes_{\mathbb{Q}[t]} \mathbb{Q}(t),
$$

and is independent of the choice of resolution. By Proposition 3.2, this gives

$$
\int_{\left[\bar{M}_{S}\right]^{\text {red }}} \frac{1}{e\left(R \pi_{*} f^{*} K_{S} \otimes \mathfrak{t}\right)} \prod_{i} A_{i}
$$

In particular we can define

$$
\mathscr{R}_{g, \beta}\left(X, \sigma_{1} \ldots \sigma_{n}\right):=\int_{\left[\bar{M}_{g, n}(S, \beta)\right]^{r e d}} \frac{1}{e\left(R \pi_{*} f^{*} K_{S} \otimes \mathfrak{t}\right)} \prod_{i=1}^{n} \operatorname{ev}_{i}^{*}\left(\sigma_{i}\right)
$$

in $\mathbb{Q}(t)$; compare $(30)$. (Throughout we use curly letters to emphasise residue invariants in $\mathbb{Z}(t)$ or $\mathbb{Q}(t)$; straight letters denote numerical invariants in $\mathbb{Z}$ or $\mathbb{Q}$.)

Setting $r:=-\operatorname{rank}\left(R \pi_{*} f^{*} K_{S}\right)=-\chi\left(f^{*} K_{S}\right)=g-1+\int_{\beta} c_{1}(S)$, we have $1 / e\left(R \pi_{*} f^{*} K_{S} \otimes t\right)=$ $t^{r}+O\left(t^{r-1}\right)$. In particular, we have the following result.

Lemma 3.3. The leading coefficient of the reduced Gromov-Witten invariants of $X$ (34) reproduces the reduced Gromov-Witten invariants of $S$ (30):

$$
\mathscr{R}_{g, \beta}\left(X, \sigma_{1} \ldots \sigma_{n}\right)=R_{g, \beta}\left(S, \sigma_{1} \ldots \sigma_{n}\right) t^{r}+O\left(t^{r-1}\right),
$$

where $r=g-1+\int_{\beta} c_{1}(S)$.

Note our controversial use of the term "leading coefficient": it is possible that this be zero but that the whole polynomial $\mathscr{R}_{g, \beta} \neq 0$.

\subsection{Reduced stable pair invariants}

The reduced obstruction theory of Theorem 2.7 restricts from $P_{n}\left(\bar{X}, \iota_{*} \beta\right)$ to endow the open set $P_{n}\left(X, \iota_{*} \beta\right)$ with a perfect obstruction theory $E_{X, r e d}^{\bullet}$. The action of $T=\mathbb{C}^{*}$ on the fibres of $X=K_{S}$ defines a $T$-action on $P_{n}\left(X, \iota_{*} \beta\right)$ with respect to which $E_{X, r e d}^{\bullet}$ is $T$-equivariant. We will define stable pair invariants of $X$ using residues and the virtual localisation formula.

As usual let $\mathbb{I}^{\bullet}:=\{\mathcal{O} \rightarrow \mathbb{F}\}$ denote the universal complex over $X \times P_{n}\left(X, \iota_{*} \beta\right)$. The universal curve (the scheme-theoretic support of $\mathbb{F}$ ) represents $c h_{2}(\mathbb{F})$. Using the usual projections $(27)$, we define the following cohomology class for each $\sigma_{i} \in H^{*}(X, \mathbb{Z})$

$$
\tau\left(\sigma_{i}\right):=\pi_{P *}\left(\operatorname{ch}_{2}(\mathbb{F}) \cdot \pi_{X}^{*}\left(\sigma_{i}\right)\right) \in H^{*}\left(P_{n}\left(X, \iota_{*} \beta\right), \mathbb{Z}\right) .
$$

For nonzero $\beta \in H_{2}(X, \mathbb{Z})$, we would like to define the stable pair invariant with insertions by

$$
\mathscr{P}_{n, \beta}^{r e d}\left(X, \sigma_{1} \ldots \sigma_{m}\right):=\int_{\left[P_{n}(X, \beta)\right]^{r e d}}\left(\prod_{i=1}^{m} \tau\left(\sigma_{i}\right)\right) .
$$




\section{Curve COUnting on SuRfaces I: TheOrY}

We make sense of this as a residue by the virtual localisation formula [GP99]:

$$
\mathscr{P}_{n, \beta}^{r e d}\left(X, \sigma_{1} \ldots \sigma_{m}\right):=\int_{\left[P_{n}(X, \beta)^{T}\right]^{r e d}} \frac{1}{e\left(N^{v i r}\right)}\left(\prod_{i=1}^{m} \tau\left(\sigma_{i}\right)\right) \in \mathbb{Z}(t) .
$$

In contrast to the Gromov-Witten case, the fixed point locus can contain pairs that are supported set-theoretically but not scheme-theoretically on $S$. However, we next check that $P_{S}=P_{n}(S, \beta)$ does provide one connected component of the fixed locus. Therefore the invariants (35) have a contribution coming entirely from $S$.

Over $P_{S} \subset P_{X}$ we slightly modify our usual notation and let $\iota_{*} \mathbb{F}$ denote the universal sheaf, where $\iota: S \hookrightarrow X$ is the inclusion of the zero-section. Then we have two universal complexes,

$$
\mathbb{I}_{S}^{\bullet}:=\left\{\mathcal{O}_{S \times P_{S}} \rightarrow \mathbb{F}\right\} \quad \text { on } S \times P_{S},
$$

and

$$
\mathbb{I}_{X}^{\bullet}:=\left\{\mathcal{O}_{X \times P_{S}} \rightarrow \iota_{*} \mathbb{F}\right\} \quad \text { on } X \times P_{S} .
$$

Proposition 3.4. The subscheme $P_{n}(S, \beta) \subset P_{n}(X, \beta)^{T}$ is both open and closed in the fixed locus. On this component, the obstruction theory $E_{X}^{\bullet}$ has fixed part

$$
\left(\left.E_{X}^{\bullet}\right|_{P_{S}}\right)^{f i x} \cong\left(R \pi_{P *} R \mathscr{H} \text { om }\left(\mathbb{I}_{S}^{\bullet}, \mathbb{F}\right)\right)^{\vee},
$$

and moving part its shifted dual

$$
\left(\left.E_{X}^{\bullet}\right|_{P_{S}}\right)^{\text {mov }} \cong R \pi_{P *} R \mathscr{H} o m\left(\mathbb{I}_{S}^{\bullet}, \mathbb{F}\right)[1] \otimes \mathfrak{t}^{*}
$$

twisted by the irreducible representation $\mathfrak{t}^{*}$ of weight -1 .

Moreover, the reduced obstruction theory $E_{X, r e d}^{\bullet} \mid P_{S}$ has the same moving part $\left(\left.E_{X}^{\bullet}\right|_{P_{S}}\right)^{\text {mov }}$ and

$$
\left(\left.E_{X, r e d}^{\bullet}\right|_{P_{S}}\right)^{\vee f i x} \cong \operatorname{Cone}\left(R \pi_{P *} R \mathscr{H} \text { om }\left(\mathbb{I}_{S}^{\bullet}, \mathbb{F}\right) \longrightarrow H^{0,2}(S) \otimes \mathcal{O}_{P_{S}}[-1]\right),
$$

where the map is obtained as the composition

$$
R \pi_{P *} R \mathscr{H} o m\left(\mathbb{I}_{S}^{\bullet}, \mathbb{F}\right) \longrightarrow R \pi_{P *} R \mathscr{H} o m(\mathbb{F}, \mathbb{F})[1] \stackrel{\operatorname{tr}}{\longrightarrow} R \pi_{P *} \mathcal{O}[1] \stackrel{\tau^{\geqslant 1}}{\longrightarrow} R^{2} \pi_{P *} \mathcal{O}[-1] .
$$

Proof. The triangle $\mathbb{I}_{X}^{\bullet} \rightarrow \mathcal{O}_{X \times P_{S}} \rightarrow \iota_{*} \mathbb{F}$ gives the following commutative diagram of exact triangles over $P_{S}$ :

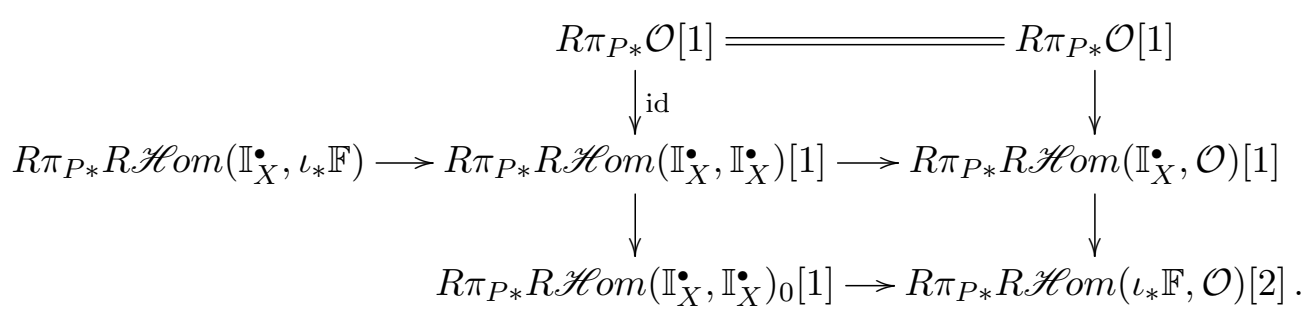

By Serre duality down $\pi_{P}: X \times P_{S} \rightarrow P_{S}$, the last term is

$$
\left(R \pi_{P *}\left(\iota_{*} \mathbb{F} \otimes \omega_{\pi_{P}}\right)\right)^{\vee}[-1] .
$$


But $\iota_{*} \mathbb{F}$ is fixed by $T$, while $\omega_{\pi_{P}}$ is just the pullback of $K_{X}$, which is trivial but with $T$-weight -1 . Therefore taking fixed parts removes this term and gives the isomorphism

$$
\left(R \pi_{P *} R \mathscr{H} o m\left(\mathbb{I}_{X}^{\bullet}, \iota_{*} \mathbb{F}\right)\right)^{f i x} \cong\left(R \pi_{P *} R \mathscr{H} o m\left(\mathbb{I}_{X}^{\bullet}, \mathbb{I}_{X}^{\bullet}\right)_{0}\right)^{\text {fix }}[1]
$$

over $P_{S} \subset P_{X}^{T}$.

Remark 3.5. The complex $\left(R \pi_{P *} R \mathscr{H} \text { om }\left(\mathbb{I}_{X}^{\bullet}, \iota_{*} \mathbb{F}\right)\right)^{\vee}$ provides the natural obstruction theory for the moduli space of stable pairs $(F, s)$. This is essentially proved in [Ill71] once one combines it with [BeF97, Theorem 4.5]: see [JS11, Sections 12.3-12.5] for a full account. However it is not perfect in general, and to define stable pair invariants one uses instead $\left(R \pi_{P *} R \mathscr{H} o m\left(\mathbb{I}_{X}^{\bullet}, \mathbb{I}_{X}^{\bullet}\right)_{0}[1]\right)^{\vee}$ [PT09]. The two theories give the same tangents, but different obstructions. Here we see that they become the same on $S \subset K_{S}$ once we pass to fixed parts.

Following [PT10, Appendix C], we next consider the exact triangle

$$
\mathbb{F} \otimes N_{S / X}^{*} \longrightarrow L \iota^{*} \mathbb{I}_{X}^{\bullet} \longrightarrow \mathbb{I}_{S}^{\bullet},
$$

where now $\iota: S \times P_{S} \hookrightarrow X \times P_{S}$. Applying $R \pi_{P *} R \mathscr{H}$ om $(\cdot, \mathbb{F})$ gives the exact triangle

$$
R \pi_{P *} R \mathscr{H} o m\left(\mathbb{I}_{S}^{\bullet}, \mathbb{F}\right) \longrightarrow R \pi_{P *} R \mathscr{H} o m\left(\mathbb{I}_{X}^{\bullet}, \iota_{*} \mathbb{F}\right) \longrightarrow R \pi_{P *} R \mathscr{H} o m\left(\mathbb{F}, \mathbb{F} \otimes K_{S}\right) .
$$

The first term has $T$-weight 0 ; the last has $T$-weight 1 . Taking fixed parts,

$$
\left(R \pi_{P *} R \mathscr{H} o m\left(\mathbb{I}_{X}^{\bullet}, \iota_{*} \mathbb{F}\right)\right)^{\text {fix }} \cong R \pi_{P *} R \mathscr{H} o m\left(\mathbb{I}_{S}^{\bullet}, \mathbb{F}\right) .
$$

Combined with (38) this gives (36).

By [GP99], the left-hand side of (36) defines a perfect obstruction theory for $P_{X}^{T}$, while the right-hand side defines one ${ }^{5}$ for $P_{S}$. Since they are isomorphic over $P_{S} \subset P_{X}^{T}$, the proof of Proposition 3.2 now shows that $P_{S} \hookrightarrow P_{X}^{T}$ is a local isomorphism, as claimed.

To derive (37) we use the Serre duality

$$
R \pi_{P *} R \mathscr{H} o m\left(\mathbb{I}_{X}^{\bullet}, \mathbb{I}_{X}^{\bullet}\right)_{0}[1] \cong\left(R \pi_{P *} R \mathscr{H} o m\left(\mathbb{I}_{X}^{\bullet}, \mathbb{I}_{X}^{\bullet} \otimes \omega_{\pi_{P}}\right)_{0}\right)^{\vee}[-2] .
$$

But $\omega_{\pi_{P}} \cong \mathcal{O} \otimes \mathfrak{t}^{*}$, so this says that

$$
\left(E_{X}^{\bullet}\right)^{\vee} \cong E_{X}^{\bullet}[-1] \otimes \mathfrak{t}
$$

on restriction to $P_{S} \subset P_{X}$. We have already seen above that $\left.E_{X}^{\bullet}\right|_{P_{S}}$ has $T$-weights only 0 and -1 , so tensoring with $\mathfrak{t}$ makes moving parts fixed and vice-versa. Taking fixed parts of (40) therefore gives (37).

Finally we have to identify the semi-regularity map on the obstruction theory. Since it is $T$-equivariant, it is only nonzero on the fixed part. Recall its definition (28) by cupping with the Atiyah class $A\left(\mathbb{I}^{\bullet}\right)$ of $\mathbb{I}^{\bullet}$ and taking the trace. From now on we work on the compactification $\bar{X}$,

\footnotetext{
${ }^{5}$ This obstruction theory - the surface analogue of that in Remark 3.5 - is perfect. This is shown in J. Otterson's Ph.D. thesis at Imperial College London using the fact that $\mathbb{F}$ has relative dimension 1 support over $P_{S}$, so $R^{\geqslant 2} \pi_{P *} \mathbb{F}=0$, which combines with the exact triangle $\mathbb{F}[-1] \rightarrow \mathbb{I}_{S}^{\bullet} \rightarrow \mathcal{O}$ to force $\mathscr{E} x t_{\pi_{P}}^{\geqslant 2}\left(\mathbb{I}_{S}^{\bullet}, \mathbb{F}\right)$ to vanish. The results of [GP99] together with (36) give a different proof of this fact.
} 


\section{CuRve COUnTing ON SURFACES I: THEORY}

so $\mathbb{I}^{\bullet}$ now denotes the complex $\left\{\mathcal{O}_{\bar{X} \times P_{S}} \rightarrow \iota_{*} \mathbb{F}\right\}$. By the naturality of the Atiyah class [BuF03, Proposition 3.11], the four left-hand squares in the following diagram commute. The right-hand square commutes because $\operatorname{tr}(a \circ b)=\operatorname{tr}(b \circ a)$. The unmarked arrows are all induced by the connecting homomorphism $\iota_{*} \mathbb{F}[-1] \rightarrow \mathbb{I}^{\bullet}$.

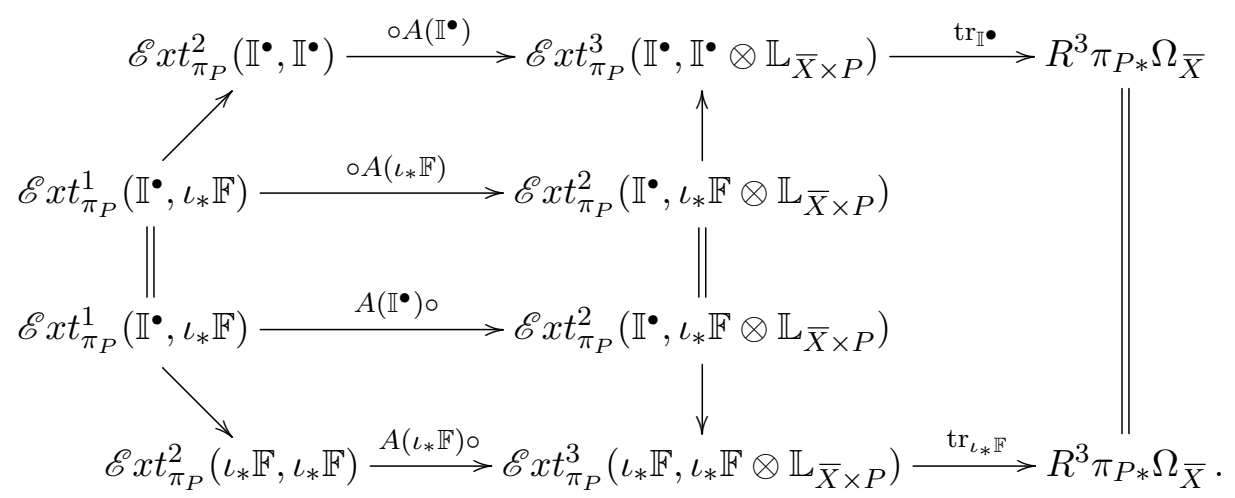

Our semi-regularity map starts with the fixed part of $\mathscr{E} x t_{\pi_{P}}^{1}\left(\mathbb{I} \bullet, \iota_{*} \mathbb{F}\right)$ on the left, takes it clockwise round the diagram to $R^{3} \pi_{P *} \Omega_{\bar{X}}$. Therefore this is the same as going anticlockwise, via $\mathscr{E} x t_{\pi_{P}}^{2}\left(\iota_{*} \mathbb{F}, \iota_{*} \mathbb{F}\right)$. By adjunction and the isomorphism $L \iota^{*} \iota_{*} \cong \mathrm{id} \oplus\left(\mathrm{id} \otimes K_{S}\right)[1]$ this is

$$
\mathscr{E} x t_{\pi_{P}}^{2}\left(\iota_{*} \mathbb{F}, \iota_{*} \mathbb{F}\right) \cong \mathscr{E} x t_{\pi_{P}}^{2}(\mathbb{F}, \mathbb{F}) \oplus \mathscr{E} x t_{\pi_{P}}^{1}\left(\mathbb{F}, \mathbb{F} \otimes K_{S}\right)
$$

We are only interested in the $T$-fixed part, that is, the first summand above.

Now, $\iota_{*} \mathbb{F} \cong q^{*} \mathbb{F} \otimes \iota_{*} \mathcal{O}_{S}$, where $q: \bar{X} \rightarrow S$ is the projection and we have omitted the pullback maps along $P$. Therefore

$$
A\left(\iota_{*} \mathbb{F}\right)=q^{*} A(\mathbb{F}) \otimes 1_{\iota_{*} \mathcal{O}_{S}}+1_{\mathbb{F}} \otimes A\left(\iota_{*} \mathcal{O}_{S}\right) .
$$

The first summand acts trivially on the first summand of (41) (since $\mathscr{E} x t_{\pi_{P}}^{3}\left(\mathbb{F},\left.\mathbb{F} \otimes \Omega_{\bar{X}}\right|_{S}\right)=0$ ). For the second summand, $A\left(\iota_{*} \mathcal{O}_{S}\right)$ lies in

$$
H^{1}\left(R \mathscr{H} o m\left(\iota_{*} \mathcal{O}_{S}, \iota_{*} \mathcal{O}_{S}\right) \otimes \Omega_{\bar{X}}\right)=H^{1}\left(\iota_{*} \mathcal{O}_{S} \otimes \Omega_{\bar{X}}\right) \oplus H^{0}\left(\iota_{*} \mathcal{O}_{S}(S) \otimes \Omega_{\bar{X}}\right)
$$

and is the canonical element of the second summand: the section $\tau$ of $T_{\bar{X}}^{*} \otimes \mathcal{O}_{S}(S)$ that projects tangent vectors to $\bar{X}$ to the normal bundle of $S \subset \bar{X}$.

So applying the second summand of (42) to the first of (41) and then taking the trace gives the upper composition in the commutative diagram

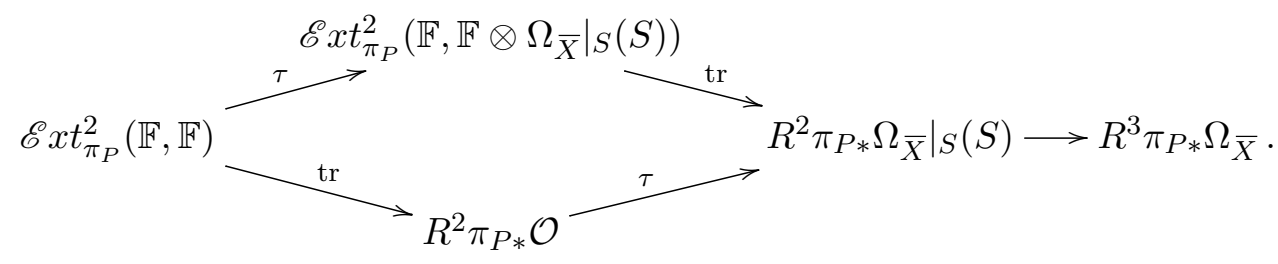

The right-hand map is most easily defined by duality: it is Serre dual to the composition 
$R^{0} \pi_{P *} \Omega_{\bar{X}}^{2} \rightarrow R^{0} \pi_{P *}\left(\left.\left.\Omega_{\bar{X}}\right|_{S} \otimes \Omega_{\bar{X}}\right|_{S}\right) \rightarrow R^{0} \pi_{P *}\left(\left.\Omega_{\bar{X}}\right|_{S}(-S)\right)$. Thus the composition $R^{2} \pi_{P *} \mathcal{O} \rightarrow$ $R^{3} \pi_{P *} \Omega_{\bar{X}}$ is an isomorphism: the pullback to $P$ of the isomorphism $\iota_{*}: H^{0,2}(S) \rightarrow H^{1,3}(\bar{X})$. Therefore the commutativity of this diagram proves the last claim of the proposition.

In particular, $P_{S}=P_{n}(S, \beta)$ carries a reduced perfect obstruction theory $\left(\left.E_{X, r e d}^{\bullet}\right|_{P_{S}}\right)^{f i x}$ and a corresponding reduced virtual cycle

$$
\left[P_{S}\right]^{r e d} \in H_{2 v}\left(P_{S}\right), \quad v:=2 h-2+n+\int_{\beta} c_{1}(S)+h^{0,2}(S),
$$

of virtual dimension $v$. Here

$$
v-h^{0,2}(S)=\operatorname{rank}\left(R \pi_{P *} R \mathscr{H} o m\left(\mathbb{I}_{S}^{\bullet}, \mathbb{F}\right)\right)=2 h-2+n+\int_{\beta} c_{1}(S) .
$$

Thus we can define the reduced invariants of $S$ to be

$$
P_{n, \beta}^{r e d}\left(S, \sigma_{1} \ldots \sigma_{m}\right):=\int_{\left[P_{n}(S, \beta)\right]^{r e d}}\left(\prod_{i=1}^{m} \tau\left(\sigma_{i}\right)\right) \in \mathbb{Z} .
$$

Secondly, we can use the virtual localisation formula to define the reduced residue invariants of $S$ to be the contribution of the component $P_{S} \subset P_{X}^{T}$ to the stable pair invariants of $X$ (35). This is

$$
\mathscr{P}_{n, \beta}^{r e d}\left(S, \sigma_{1} \ldots \sigma_{m}\right):=\int_{\left[P_{n}(S, \beta)\right]^{\text {red }}} \frac{1}{e\left(N^{v i r}\right)}\left(\prod_{i=1}^{m} \tau\left(\sigma_{i}\right)\right) \in \mathbb{Z}(t),
$$

where $N^{\text {vir }}=\left(R \pi_{P *} R \mathscr{H} \text { om }\left(\mathbb{I}_{S}^{\bullet}, \mathbb{F}\right)\right)^{\vee}[-1] \otimes \mathfrak{t}$. Thus

$$
1 / e\left(N^{v i r}\right)=t^{r}+O\left(t^{r-1}\right)
$$

where $r:=\operatorname{rank}\left(R \pi_{P *} R \mathscr{H}\right.$ om $\left.\left(\mathbb{I}_{S}^{\bullet}, \mathbb{F}\right)\right)=v-h^{0,2}(S)$, and

$$
\mathscr{P}_{n, \beta}^{r e d}\left(S, \sigma_{1} \ldots \sigma_{m}\right)=\left(P_{n, \beta}^{r e d}\left(S, \sigma_{1} \ldots \sigma_{m}\right)\right) t^{r}+O\left(t^{r-1}\right) .
$$

That is, the residue invariants contain as their leading coefficient the reduced stable pair invariants of $S$; compare Lemma 3.3. Of course it is often the case (for degree reasons, for instance) that the latter vanishes while the former does not. We will see an example of this in Section 5.

Under certain circumstances, $P_{S}$ is all of $P_{X}^{T}$, so that $\mathscr{P}_{n, \beta}^{r e d}(S)=\mathscr{P}_{n, \beta}^{r e d}(X)$. The following proposition, proved in the sequel [KT14], gives examples of this. We will not use this result in the current paper. Recall [BS91] that a line bundle $L$ on $S$ is said to be $k$-very ample if $H^{0}(L) \rightarrow H^{0}\left(\left.L\right|_{Z}\right)$ is surjective for every length $k+1$ subscheme $Z$ of $S$. We say that $\beta$ is $k$-very ample if there exists a line bundle in $\operatorname{Pic}_{\beta}(S)$ which is $k$-very ample.

Proposition 3.6. [KT14, Proposition 5.1] In the following two cases there is an isomorphism $P_{n}\left(X, \iota_{*} \beta\right)^{T} \cong P_{n}(S, \beta)$ :

$-\beta$ is irreducible, or

$-K_{S}^{-1}$ is nef, $\beta$ is $(2 \delta+1)$-very ample and $n \leqslant 1-h+\delta$. 


\section{Curve COUNTing on SURFACES I: THEORY}

Here $h$ is the arithmetic genus of curves in class $\beta$, determined by $2 h-2=\beta^{2}-c_{1}(S) \cdot \beta$. The inequality on $n$ means the stable pairs have at most $\delta$ free points.

The previous proposition is false for arbitrary surfaces. For instance, if $K_{S}=\mathcal{O}_{S}\left(C_{0}\right)$ is effective, then consider $\beta=n C_{0}$ and let $C$ be the $n$-fold thickening of $C_{0}$ along the fibres of $K_{S}$. This is $T$-fixed with $\chi=1-h$, but not scheme-theoretically supported on $S$. However one can often make it true again by restricting to small linear subsystems in the space of curves. We will do this in Section 5.

\section{Insertions and linear systems}

\subsection{Det and div}

Let $\operatorname{Hilb}_{\beta}(S)$ denote the Hilbert scheme of curves in $S$ in class $\beta$. These are subschemes $Z \subset S$ of Hilbert polynomial $\chi\left(\mathcal{O}_{Z}(n)\right)=\frac{1}{2} \int_{\beta} c_{1}(S)-\beta^{2} / 2+n \int_{\beta} c_{1}(\mathcal{O}(1))$ for every ample line bundle $\mathcal{O}(1)$ on $S$. In contrast to the threefold case, these are all pure curves, that is, subschemes of pure dimension one, with no free or embedded points. Such a curve $C$ is a divisor with an associated line bundle $\mathcal{O}(C)$, defining an Abel-Jacobi map $A J: \operatorname{Hilb}_{\beta}(S) \rightarrow \operatorname{Pic}_{\beta}(S)$. Both of these spaces receive maps from both of our moduli spaces of curves in $S$ :

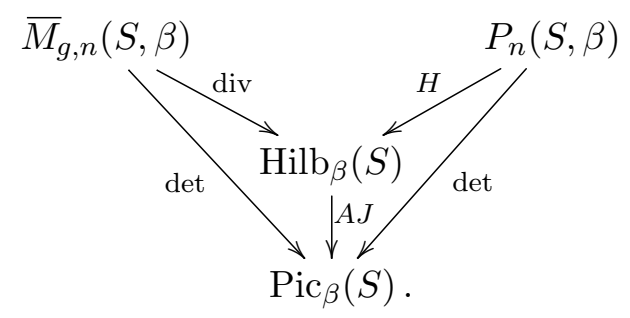

The map div takes a stable map to its divisor class, which is its image with multiplicities. That is, if the irreducible components $C_{i}$ of its image are multiply covered $k_{i}$ times then the image is the divisor $\sum_{i} k_{i} C_{i}$ defined by the ideal sheaf $\bigotimes_{i} \mathscr{I}_{C_{i}}^{k_{i}}$. This set theoretic map can be made into a morphism by taking a stable map $f: C \rightarrow S$ to the line bundle $\operatorname{det}\left(f_{*} \mathcal{O}_{C}\right) \in \operatorname{Pic}_{\beta}(S)$ and its canonical section [KM76].

The map $H$ takes a pair $(F, s)$ to the scheme theoretic support of $F$. In fact, it is proved in [PT10, Proposition B.8] that $P_{n}(S, \beta)$ is a relative Hilbert scheme of points on the fibres of the universal curve over $\operatorname{Hilb}_{\beta}(S)$.

The fibre of $A J$ over the line bundle $L$ is the full linear system $\mathbb{P}\left(H^{0}(L)\right)$. If we wish to derive invariants from just one such linear system, we can do so using insertions as in [BL99]. We reprove their results in a slightly simpler way.

Picking a base point in $\mathrm{Pic}:=\operatorname{Pic}_{\beta}(S)$ gives a canonical isomorphism

$$
\mathrm{Pic} \cong H^{1}(S, \mathbb{R}) / H^{1}(S, \mathbb{Z}) \text {. }
$$

Therefore $H^{1}(\mathrm{Pic}, \mathbb{R})$ is canonically isomorphic to the space $H^{1}(S, \mathbb{R})^{*}$ of constant one-forms 
on Pic. Via the isomorphism $H_{1}(S, \mathbb{R}) \cong H^{1}(S, \mathbb{R})^{*}$, a cycle $\gamma \in H_{1}(S)$ gets taken to the constant one-form $\tilde{\gamma}$ whose pairing with a constant tangent vector $v \in H^{1}(S, \mathbb{R})$ to Pic is

$$
\langle\tilde{\gamma}, v\rangle_{\operatorname{Pic}}=\int_{\gamma} v
$$

where the right-hand integral takes place on $S$.

Pick a Poincaré line bundle $\mathcal{P}$ on $S \times$ Pic, and let $\pi_{S}, \pi_{\text {Pic }}$ denote the obvious projections.

Lemma 4.1. We have $\tilde{\gamma}=\pi_{\operatorname{Pic} *}\left(\pi_{S}^{*}([\gamma]) \cup c_{1}(\mathcal{P})\right)$, where $[\gamma]:=\operatorname{PD}(\gamma) \in H^{3}(S)$ is the Poincaré dual of $\gamma$.

Proof. Consider the Künneth component of $c_{1}(\mathcal{P}) \in H^{2}(S \times$ Pic $)$ in

$$
H^{1}(S) \otimes H^{1}(\mathrm{Pic}) \cong \operatorname{Hom}\left(H_{1}(S), H^{1}(\mathrm{Pic})\right) .
$$

Identifying the right-hand side of (47) with

$$
\operatorname{Hom}\left(H_{1}(S), H_{1}(S)\right),
$$

its class is the identity. Thus, considered as an element of $\operatorname{Hom}\left(H_{1}(S), H^{1}(\mathrm{Pic})\right)$, it takes $\gamma \in$ $H_{1}(S)$ to $\tilde{\gamma} \in H^{1}(\mathrm{Pic})$, since this is what maps back to $\gamma$ under $H^{1}(\mathrm{Pic}) \cong H_{1}(S)$.

However, via the isomorphism $(47), c_{1}(\mathcal{P})$ takes $\gamma$ to

$$
\pi_{\mathrm{Pic} *}\left(\left.c_{1}(\mathcal{P})\right|_{\gamma \times \operatorname{Pic}}\right)=\pi_{\mathrm{Pic} *}\left(c_{1}(\mathcal{P}) \cup \operatorname{PD}(\gamma \times \mathrm{Pic})\right),
$$

which is $\pi_{\mathrm{Pic} *}\left(c_{1}(\mathcal{P}) \cup \pi_{S}^{*}[\gamma]\right)$, as required.

The following is a result of Bryan and Leung [BL99, Theorem 2.1].

Proposition 4.2. The cohomology class $\pi_{*} f^{*}[\gamma]$ is $\operatorname{det}^{*} \tilde{\gamma}$.

Proof. We work with the diagram

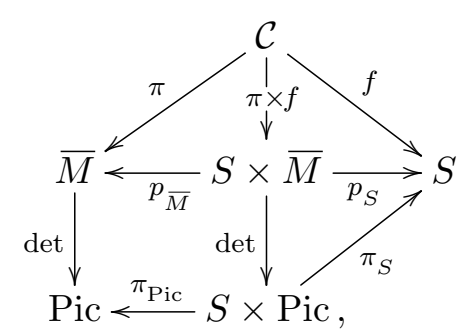

where $\mathcal{C}$ is the universal curve. On $S \times \bar{M}$, the divisor class $\operatorname{div} \mathcal{C}$ defines a line bundle $\mathcal{O}_{S \times \bar{M}}(\operatorname{div} \mathcal{C})$ which, on restriction to any $S$-fibre, is isomorphic to the restriction of the pullback det* $\mathcal{P}$ of the Poincaré line bundle. The two therefore differ only by a line bundle pulled back from $\bar{M}$ (that 


\section{Curve COUnting on SURFACES I: THEORY}

is, with no $S$-component). Thus for degree reasons we have

$$
\begin{aligned}
\operatorname{det}^{*} \tilde{\gamma} & =\operatorname{det}^{*} \pi_{\operatorname{Pic} *}\left(\pi_{S}^{*}[\gamma] \cup c_{1}(\mathcal{P})\right) \\
& =p_{\bar{M} *} \operatorname{det}^{*}\left(\pi_{S}^{*}[\gamma] \cup c_{1}(\mathcal{P})\right) \\
& =p_{\bar{M} *}\left(p_{S}^{*}[\gamma] \cup[\operatorname{div} \mathcal{C}]\right) \\
& =p_{\bar{M} *}\left(\left.\left(p_{S}^{*}[\gamma]\right)\right|_{\operatorname{div} \mathcal{C})}\right. \\
& =\pi_{*}(\pi \times f)^{*}\left(p_{S}^{*}[\gamma]\right) \\
& =\pi_{*} f^{*}[\gamma]
\end{aligned}
$$

where in the penultimate line we have used the fact that the fundamental class of $\operatorname{div} \mathcal{C}$ is the same as that of $\mathcal{C}$ pushed forward by $\pi \times f$.

Following Bryan and Leung [BL99], let $\gamma_{i}, i=1, \ldots, b_{1}=b_{1}(S)$ be an integral basis of $H_{1}(S, \mathbb{Z}) /$ torsion, oriented so that

$$
\int_{\text {Pic }} \tilde{\gamma}_{1} \wedge \ldots \wedge \tilde{\gamma}_{b_{1}}=1
$$

Thus $\tilde{\gamma}_{1} \wedge \ldots \wedge \tilde{\gamma}_{b_{1}}=[p t]$ is Poincaré dual to a point of Pic, so by Proposition 4.2 and $b_{1}$ applications of (32),

$$
\operatorname{det}^{*}([p t])=r_{*}\left(\operatorname{ev}_{1}^{*}\left[\gamma_{1}\right] \wedge \ldots \wedge \operatorname{ev}_{b_{1}}^{*}\left[\gamma_{b_{1}}\right]\right)
$$

where $r: \bar{M}_{g, n+b_{1}} \rightarrow \bar{M}_{g, n}$ is the map that forgets the last $b_{1}$ points and stabilises.

Since $r$ intertwines $\mathrm{ev}_{1}, \ldots, \mathrm{ev}_{n}$ on $\bar{M}_{g, n+b_{1}}(S, \beta)$ and $\bar{M}_{g, n}(S, \beta)$, when we apply this to the reduced Gromov-Witten invariants (30) we get

$$
\begin{aligned}
R_{g, \beta}\left(S, \sigma_{1}\right. & \left.\ldots \sigma_{n}\left[\gamma_{1}\right] \ldots\left[\gamma_{b_{1}}\right]\right) \\
& =\int_{\left[\bar{M}_{g, n}(S, \beta)\right]^{r e d}}\left(\operatorname{ev}_{1}^{*} \sigma_{1} \wedge \ldots \wedge \operatorname{ev}_{n}^{*} \sigma_{n}\right) \wedge \operatorname{det}^{*}([p t]) \\
& =\int_{j^{!}\left[\bar{M}_{g, n}(S, \beta)\right]^{r e d}} \operatorname{ev}_{1}^{*} \sigma_{1} \wedge \ldots \wedge \operatorname{ev}_{n}^{*} \sigma_{n} .
\end{aligned}
$$

Here $j !$ is the refined Gysin map [Ful98, Section 6.2] for the inclusion of a point $j:\{L\} \hookrightarrow \operatorname{Pic}_{\beta}(S)$.

It would be nice to write $j^{!}\left[\bar{M}_{g, n}(S, \beta)\right]^{\text {red }}$ in the form $\left[\bar{M}_{g, n}(S,|L|)\right]^{\text {red }}$. That is, we would like to see it as a reduced virtual cycle on the moduli space of stable maps whose associated divisor lies in the linear system $|L|$, defined by the Cartesian diagram

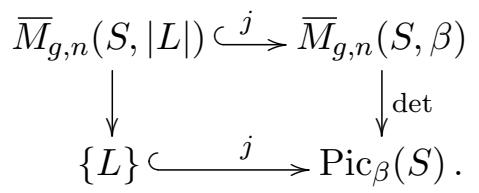


Now we have the diagram

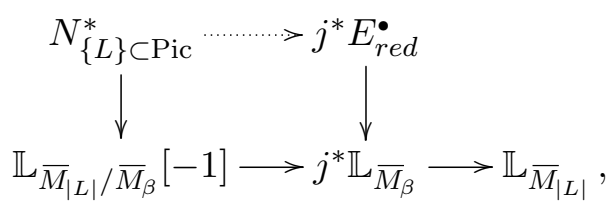

with $\left.N_{\{L\} \subset \text { Pic }}^{*} \cong \Omega_{\text {Pic }}\right|_{\{L\}} \cong H^{1}\left(K_{S}\right)$ the conormal bundle to the point $L$ in Pic. If we can fill in the dotted arrow, its cone is easily seen to give the required reduced perfect obstruction theory for $\bar{M}_{|L|}=\bar{M}_{g, n}(S,|L|)$.

The arrow is produced by simply repeating Section 2.2 for $H^{1}\left(K_{S}\right)$ in place of $H^{0}\left(K_{S}\right)$, giving

$$
H^{1}\left(K_{S}\right) \otimes \mathcal{O}_{\bar{M}_{|L|}} \longrightarrow j^{*} E_{r e d}^{\bullet}
$$

analogously to the map $H^{0}\left(K_{S}\right) \otimes \mathcal{O}_{\bar{M}_{\beta}} \longrightarrow E^{\bullet}[-1]$ of (15). However checking that the resulting diagram (52) commutes appears technically difficult. We hope to return to this issue in the future.

\subsection{Point insertions and linear subsystems}

Having cut down to stable maps with image in a single linear system using insertions (50), we next show how point insertions correspond (at the level of virtual cycles) to cutting down to linear subsystems.

We work with the commutative diagram

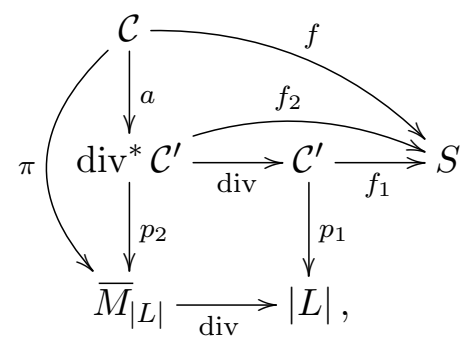

where $\mathcal{C}^{\prime} \rightarrow|L|$ is the universal curve over the linear system $|L|$ and $\pi: \mathcal{C} \rightarrow \bar{M}_{|L|}$ is the universal curve over the space of stable maps. Over $\bar{M}_{|L|}$ the latter maps to the former, contracting some components and replacing multiple covers by scheme-theoretic multiplicities, carrying the fundamental class of one to the other. In particular, $p_{2}^{*}=a_{*} \pi^{*}$ on homology.

Concentrating on the bottom right-hand corner, we show first that

$$
p_{1 *} f_{1}^{*}([p t])=h,
$$

where $[p t] \in H^{4}(S)$ is the Poincaré dual of a point of $S$, and $h \in H^{2}(|L|)$ is the hyperplane class. 


\section{Curve COUNTING ON SURFACES I: THEORY}

This follows from the computation

$$
\begin{aligned}
\int_{|L|} p_{1 *} f_{1}^{*}([p t]) h^{\operatorname{dim}|L|-1} & =\int_{\mathbb{P} 1} p_{1 *} f_{1}^{*}([p t])=\int_{\mathcal{C}_{\mathbb{P} 1}^{\prime}} f_{1}^{*}([p t]) \\
& =\operatorname{deg}\left(\left.f_{1}\right|_{\mathcal{C}_{\mathbb{P}}^{\prime}}: \mathcal{C}_{\mathbb{P} 1}^{\prime} \rightarrow S\right)=1 .
\end{aligned}
$$

Here $\mathbb{P}^{1} \subset|L|$ is any pencil, with universal curve $\mathcal{C}_{\mathbb{P}^{1}}^{\prime}$ over it. Since any pencil of curves sweeps out $S$, the map $\mathcal{C}_{\mathbb{P}^{1}}^{\prime} \rightarrow S$ is birational and thus has degree 1 , as claimed.

From (53) and (54) it follows that

$$
p_{2 *} f_{2}^{*}([p t])=p_{2 *} \operatorname{div}^{*} f_{1}^{*}([p t])=\operatorname{div}^{*} p_{1 *} f_{1}^{*}([p t])=\operatorname{div}^{*}(h) .
$$

Now $p_{2}^{*}=a_{*} \pi^{*}$ on homology implies that $p_{2 *}=\pi_{*} a^{*}$ on cohomology. Therefore

$$
\operatorname{div}^{*}(h)=\pi_{*} a^{*} f_{2}^{*}([p t])=\pi_{*} f^{*}([p t]) .
$$

Thus we get the point insertion $\pi_{*} f^{*}([p t])$. Repeating $m$ times gives the $m$-point insertions as in (32). In particular we get from (50) that

$$
R_{g, \beta}\left(S, \sigma_{1} \ldots \sigma_{n}\left[\gamma_{1}\right] \ldots\left[\gamma_{b_{1}}\right][p t]^{m}\right)=\int_{i^{!} j^{!}\left[\bar{M}_{g, n}(S, \beta)\right]^{r e d}} \operatorname{ev}_{1}^{*} \sigma_{1} \wedge \ldots \wedge \mathrm{ev}_{n}^{*} \sigma_{n} .
$$

As before $j^{!}$is the generalised Gysin map for the inclusion $j:\{L\} \hookrightarrow \operatorname{Pic}_{\beta}$, and we let $i$ be the inclusion of a codimension $m$ linear subsystem of $|L|$, where $m$ is the number of point insertions. So the above is an integral over the space of stable maps mapping to this linear subsystem.

\subsection{Extension to pairs and the threefold $X$}

We have concentrated on the moduli space of stable maps $\bar{M}_{g, n}(S, \beta)$, but the above results about insertions apply equally to $P_{n}(S, \beta)$. The proofs are the same (slightly easier even, since the universal curve, which in the stable pairs case is $c_{2}(\mathbb{F})$, embeds into $S \times P$ in the diagram analogous to (48), and the map $a$ is an isomorphism in (53)). The upshot is the following analogue of (56):

$$
P_{n, \beta}^{r e d}\left(S, \sigma_{1}, \ldots \sigma_{m}\left[\gamma_{1}\right] \ldots\left[\gamma_{b_{1}}\right][p t]^{k}\right)=\int_{i^{\prime} j^{!}\left[P_{n}(S, \beta)\right]^{r e d}} \prod_{i=1}^{m} \tau\left(\sigma_{i}\right),
$$

with $i$ and $j$ as before. The reduced obstruction theory of Appendix 5.2 is easily done relative to $\operatorname{Pic}_{\beta}(S)$ so the analogue of the commutative diagram (52) is automatic. Therefore (though we will not need or use it) the right-hand side of (57) can equally be written as an integral over a reduced virtual cycle for $P_{n}(S, \mathbb{P}):=P_{n}(S,|L|) \times_{|L|} \mathbb{P}$, where $i: \mathbb{P} \hookrightarrow|L|$ is the linear subsystem.

With the obvious modifications the results also apply to $X=K_{S}$, and to the corresponding residue invariants. By pushing down curves in $X$ to $S$ before applying div (46) we get maps from $P_{n}\left(X, \iota_{*} \beta\right)$ and $\bar{M}_{g, n}\left(X, \iota_{*} \beta\right)$ to $\operatorname{Hilb}_{\beta}(S)$ and $\operatorname{Pic}_{\beta}(S)$. Proposition 4.2 then holds with $[\gamma]$ replaced by its pullback to $X$, and the same therefore applies to formula (50). 
Similarly, formula (55) also holds on $X$ when $[p t]$ is replaced by its pullback to $X$, that is, by the Poincaré dual of a fibre of $K_{S}$. The same then applies to formula (56).

\section{Counting nodal curves}

\subsection{Severi degrees as reduced Gromov-Witten invariants}

In this section we show that the Severi degrees (as studied by Göttche [Got98], for instance) counting nodal curves in very ample linear systems can be seen as a special case of the reduced Gromov-Witten invariants (30). In particular we give these classical invariants a more modern treatment using virtual cycles, allowing us to extend them to virtual counts outside of the very ample regime.

Fix a line bundle $L$ with $H^{1}(L)=0$ (which almost certainly follows from the ampleness assumptions below) and $c_{1}(L)=\beta$ satisfying condition (3); in particular then $H^{2}(L)=0$ also. Given a curve $C$, we let $g(C)$ denote its arithmetic genus, defined by $1-g(C):=\chi\left(\mathcal{O}_{C}\right)$ even when it is not reduced or connected. When $C$ is reduced its geometric genus $\bar{g}(C)$ is defined to be $g(\bar{C})$, the genus of its normalisation. Finally, let $h$ denote the arithmetic genus of curves in $|L|$, so that $2 h-2=\beta^{2}-c_{1}(S) \cdot \beta$.

Proposition 5.1. If $L$ is a $(2 \delta+1)$-very ample line bundle on $S$ then the general $\delta$-dimensional linear system $\mathbb{P}^{\delta} \subset|L|$ contains a finite number of irreducible $\delta$-nodal curves appearing with multiplicity one, and all other curves are reduced and irreducible with geometric genus $\bar{g}>h-\delta$.

Proof. This result without the irreducibility requirement is proved in [KST11, Proposition 2.1] under the weaker assumption of $\delta$-very ampleness.

So to finish we assume for a contradiction that there exists a reducible curve in $\mathbb{P}^{\delta} \subset|L|$. Since it must be reduced, we can write it as $A+B$, with $A$ and $B$ nonzero and having no common irreducible components.

By the Hodge index theorem, $A^{2} \leqslant(L \cdot A)^{2} / L^{2}$ for any positive $L \in H^{1,1}(S)$ and arbitrary $A \in H^{1,1}(S)$. (Proof: $A-(L \cdot A) L / L^{2}$ is orthogonal to $L$ so has square $\leqslant 0$.) Applied to our situation we get

$$
A \cdot B=A \cdot(L-A) \geqslant A \cdot L-\frac{(A \cdot L)^{2}}{L^{2}}=\frac{(A \cdot L)(B \cdot L)}{L^{2}} .
$$

By symmetry we may assume that $A \cdot L \leqslant B \cdot L$. Then

$$
L^{2}=A \cdot L+B \cdot L \leqslant 2 B \cdot L
$$

so that by (58),

$$
A \cdot B \geqslant \frac{A \cdot L}{2} \geqslant \frac{2 \delta+1}{2}
$$

The inequality $A \cdot L \geqslant 2 \delta+1$ follows from the $(2 \delta+1)$-very ampleness of $L$ as follows. It suffices to show the inequality for any irreducible effective divisor $A$. Choose $2 \delta+2$ smooth points on $A$. 


\section{Curve COUNTING ON SURFACES I: THEORY}

By the definition of $(2 \delta+1)$-very ampleness, there is a divisor in $|L|$ which passes through the first $2 \delta+1$ points, but not the last one. Therefore the divisor does not contain $A$, and $L \cdot A \geqslant 2 \delta+1$, as required.

But (59) implies the normalisation of $A+B$ pulls apart more than $\delta$ intersection points, which makes the geometric genus of $A+B$ less than $h-\delta$, a contradiction.

We call these irreducible $\delta$-nodal curves

$$
D_{i}, \quad i=1, \ldots, n_{\delta}(L) .
$$

Here $n_{\delta}(L)$ is the intersection of $\mathbb{P}^{\delta}$ with the Severi variety

$$
\overline{\{C \in|L|: C \text { has } \delta \text { nodes }\}} \subset|L|,
$$

that is, it is the Severi degree (4) studied by Göttsche [Got98].

Since they are irreducible, the normalisation maps $\bar{D}_{i} \rightarrow D_{i}$ define stable maps $f_{i}: \bar{D}_{i} \rightarrow S$ from smooth connected curves. In fact, these are all of the points of $\bar{M}_{h-\delta}\left(S, \mathbb{P}^{\delta}\right):=\operatorname{div}^{-1}\left(\mathbb{P}^{\delta}\right)$.

Proposition 5.2. Let $L$ be a $(2 \delta+1)$-very ample line bundle on $S$. The only points of $\bar{M}_{h-\delta}(S, \beta)$ whose divisor class lies in $\mathbb{P}^{\delta}$ are the normalisations of the $\delta$-nodal curves $D_{i}$. These are smooth points of $\bar{M}_{h-\delta}(S, \beta)$ and smooth isolated points of $\bar{M}_{h-\delta}\left(S, \mathbb{P}^{\delta}\right)$.

Proof. Choose a stable map $f: C \rightarrow S$ whose divisor class lies in $\mathbb{P}^{\delta}$. So $C$ is connected and at worst nodal. By Proposition 5.1 its image $\Sigma:=f_{*} C$ is reduced and irreducible of geometric genus at least $h-\delta$, and $f$ is generically one-to-one except on contracted irreducible components.

Let $C_{1}, \ldots C_{k}$ denote the irreducible components of $C$ which are contracted, and let $C_{k+1}$ denote the one which surjects onto $\Sigma$. Therefore $\bar{\Sigma}=\bar{C}_{k+1}$ and $\bar{C}=\bar{C}_{1} \sqcup \ldots \sqcup \bar{C}_{k+1}$.

We want to show that $g(C) \geqslant h-\delta$ with equality only if $(C, f)$ is the normalisation of one of the $\delta$-nodal curves $D_{i}$. We have

$$
g(C)-1=\sum_{i=1}^{k+1}\left(g\left(\bar{C}_{i}\right)-1\right)+d,
$$

where $d=g(C)-g(\bar{C})$ is the number of nodes of $C$, and

$$
g(\bar{\Sigma})-1=g\left(\bar{C}_{k+1}\right)-1 .
$$

Thus

$$
g(C)-g(\bar{\Sigma})=\sum_{i=1}^{k}\left(g\left(\bar{C}_{i}\right)-1\right)+d .
$$

Contracted components of genus $g\left(\bar{C}_{i}\right) \geqslant 2$ contribute strictly positively to (61). Contracted components of genus one must contain one of the nodes of $C$ by stability (or connectedness), forcing $d>0$ and therefore also contributing strictly positively to (61). Finally, contracted projective lines $\mathbb{P}^{1}$ must contain at least three of the nodes in $C$, by stability. So if there are 
$p>0$ contracted projective lines $\mathbb{P}^{1}$ then there must be at least $3 p$ preimages of nodes upstairs on $\bar{C}$ and so at least $3 p / 2$ nodes downstairs on $C$. In particular, $d \geqslant 3 p / 2>p$, so again (61) is strictly positive.

It follows that

$$
g(C) \geqslant g(\bar{\Sigma}) \geqslant h-\delta
$$

with equality implying that there are no contracted components, no nodes $(d=0)$, and, by Proposition 5.1, $\Sigma$ must be one of the $\delta$-nodal curves $D_{i}$. Thus $C$ is smooth and $f$ is the normalisation of the image $D_{i}$.

Finally, we deal with the deformation theory of a nodal curve $D \subset S$. The "multiplicity one" statement of Proposition 5.1 refers to the scheme structure on the locus of nodal curves defined by locally pulling back the reduced scheme structure from the miniversal deformation space of the singularity [KST11, proof of Proposition 2.1]. What is in fact proved is that the locus of $\delta$-nodal curves is smooth of codimension $\delta$, and then $\mathbb{P}^{\delta}$ is chosen transverse to it. Equivalently, the composition

$$
T_{D} \mathbb{P}^{\delta} \rightarrow H^{0}\left(\mathcal{O}_{D}(D)\right) \rightarrow H^{0}\left(\mathcal{O}_{Z}(D)\right)
$$

is surjective. Here $Z \subset D$ is the singular set of $D$ - the union of its nodes - and $H^{0}\left(\mathcal{O}_{Z}(D)\right)$ is its miniversal deformation space.

We want to relate this to the deformation theory of the resulting stable map $f: \bar{D} \rightarrow S$ given by normalising $D$. Since $f$ is an immersion, $T_{\bar{D}} \rightarrow f^{*} T_{S}$ is an injection; we define $N_{f}$ to be its cokernel. By local calculation,

$$
f_{*} N_{f} \cong \mathscr{I}_{Z}(D) \subset \mathcal{O}_{D}(D),
$$

where $Z \subset D$ is the union of the nodes of $D$. The usual stable map deformation-obstruction theory (12) reduces in this case to

$$
\left(E^{\bullet}\right)^{\vee}=R \Gamma\left(T_{\bar{D}} \rightarrow f^{*} T_{S}\right)=R \Gamma\left(\bar{D}, N_{f}\right)=R \Gamma\left(D, f_{*} N_{f}\right)=R \Gamma\left(D, \mathscr{I}_{Z}(D)\right) .
$$

In particular, the vector space of first order deformations is $H^{0}\left(\mathscr{I}_{Z}(D)\right)$, and those with divisor class in $\mathbb{P}^{\delta}$ are given by the intersection in $H^{0}\left(\mathcal{O}_{D}(D)\right)$ of $T_{D} \mathbb{P}^{\delta}$ and $H^{0}\left(\mathscr{I}_{Z}(D)\right)$. That is, the Zariski tangent space to $\bar{M}_{h-\delta}\left(S, \mathbb{P}^{\delta}\right)$ at $(D, f)$ is the kernel of the composition (62). But that map is surjective between vector spaces of dimension $\delta$, so has kernel 0 . Therefore the $\left(\bar{D}_{i}, f_{i}\right) \in \bar{M}_{h-\delta}\left(S, \mathbb{P}^{\delta}\right)$ are isolated points as claimed.

From this also follows the fact that $\bar{M}_{h-\delta}(S, \beta)$ is smooth at $\left(\bar{D}_{i}, f_{i}\right)$. In fact, let $d$ denote the dimension of its Zariski tangent space at $\left(\bar{D}_{i}, f_{i}\right)$, and let $v$ be its virtual dimension (17) in the reduced obstruction theory. As for any space with perfect obstruction theory, we have

$$
d \geqslant v
$$

with equality only if $\bar{M}_{h-\delta}(S, \beta)$ is smooth at $\left(\bar{D}_{i}, f_{i}\right)$. Cutting down by $h^{0,1}(S)$ equations to $\bar{M}_{h-\delta}(S,|L|)$ and then by a further $\chi(L)-1-\delta$ equations to $\bar{M}_{h-\delta}\left(S, \mathbb{P}^{\delta}\right)$, the Zariski tangent space has complex dimension at least

$$
v-h^{0,1}(S)-\chi(L)+1+\delta=0 .
$$




\section{Curve COUnting on SuRfaces I: TheOrY}

But the $\left(\bar{D}_{i}, f_{i}\right)$ are isolated in $\bar{M}_{h-\delta}\left(S, \mathbb{P}^{\delta}\right)$, so the above inequalities are both equalities, and the $\left(\bar{D}_{i}, f_{i}\right)$ indeed define smooth points of $\bar{M}_{h-\delta}(S, \beta)$.

(Alternatively one can compute by the deformation theory above that the reduced obstruction space vanishes.)

Combining this with (56) we find that the reduced Gromov-Witten invariants with insertions

equal

$$
(\ldots):=\left(S,\left[\gamma_{1}\right] \ldots\left[\gamma_{b_{1}(S)}\right][p t]^{\chi(L)-1-\delta}\right)
$$

$$
R_{h-\delta, \beta}(\ldots)=n_{\delta}(L) .
$$

Göttsche conjectured that the numbers $n_{\delta}(L)$ should be degree $\delta$ polynomials in the four numbers $L^{2}, K_{S} \cdot L, K_{S}^{2}, c_{2}(S)$ when $L$ is at least $(5 \delta-1)$-very ample. This was proved by Tzeng [Tze12], and later in [KST11] for $L$ at least $\delta$-very ample using the stable pair methods of the next section.

By Lemma 3.3 the reduced residue Gromov-Witten invariants of $X$ contain the same numbers. (When working on $X$ we use the insertions

$$
(\ldots):=\left(X, q^{*}\left[\gamma_{1}\right] \ldots q^{*}\left[\gamma_{b_{1}(S)}\right] q^{*}[p t]^{\chi(L)-1-\delta}\right)
$$

pulled back from (63) by the projection $q: X \rightarrow S$.) In fact, for degree reasons the other terms in Lemma 3.3 all vanish. Summarising then, we have the following result.

Theorem 5.3. Let $L$ be a $(2 \delta+1)$-very ample line bundle with $H^{1}(L)=0$ and $c_{1}(L)=\beta$ satisfying condition (3). Then the reduced Gromov-Witten invariants of both $(S, \beta)$ and $(X=$ $\left.K_{S}, \iota_{*} \beta\right)$ include the Severi degrees $n_{\delta}(L)$ of $\delta$-dimensional linear systems in $|L|$ :

$$
\begin{aligned}
& R_{h-\delta, \beta}(\ldots)=n_{\delta}(L), \\
& \mathscr{R}_{h-\delta, \beta}(\ldots)=n_{\delta}(L) \cdot t^{h-\delta-1+\int_{\beta} c_{1}(S)} .
\end{aligned}
$$

Here (...) denotes either of the insertions (63) on $S$ or (64) on $X$, and $n_{\delta}(L)(60)$ is a universal degree $\delta$ polynomial in $\beta^{2}, \int_{\beta} c_{1}(S), c_{1}(S)^{2}$ and $c_{2}(S)$.

Therefore $R_{h-\delta, \beta}\left(S,\left[\gamma_{1}\right] \ldots\left[\gamma_{b_{1}(S)}\right][p t]^{\chi(L)-1-\delta}\right)$ gives us one way of extending Severi degrees to the case when $L$ is not very ample, and has possibly nonvanishing $H^{1}$. It is a virtual count of irreducible $\delta$-nodal curves satisfying the incidence conditions.

\subsection{Severi degrees as reduced stable pair invariants}

One can encode the reduced residue Gromov-Witten invariants $\mathscr{R}_{g, \beta}$ in BPS form. By Proposition 5.1 all curves in $\mathbb{P}^{\delta}$ are reduced and irreducible. In particular, the stable maps in $\bar{M}_{g}\left(S, \mathbb{P}^{\delta}\right)$ all have irreducible image and involve no multiple covers. So the universal formula for the BPS invariants $r_{g, \beta} \in \mathbb{Q}(t)$ reduces to

$$
\sum_{g=0}^{\infty} \mathscr{R}_{g, \beta}(\ldots) u^{2 g-2}=\sum_{g=0}^{\infty} r_{g, \beta}(\ldots)(2 \sin u / 2)^{2 g-2},
$$


where we use the same insertions (...) as above $(63,64)$. Via the Gopakumar-Vafa, MNOP and stable pairs conjectures [GV98, MNOP06, PT09], all extended to the reduced and equivariant cases, the $r_{g, \beta}$ defined by (65) should lie in $\mathbb{Z}(t)$ and can also be calculated via universal formulae in the reduced residue stable pair invariants.

The leading $u^{2 h-2 \delta-2}$-term in (65) states that in genus $h-\delta$, the reduced BPS invariants are just the reduced residue Gromov-Witten invariants, which by Lemma 3.3 are the reduced Gromov-Witten invariants up to a shift in the equivariant parameter:

$$
\begin{aligned}
r_{h-\delta, \beta}(\ldots)=\mathscr{R}_{h-\delta, \beta}(\ldots) & =R_{h-\delta, \beta}(\ldots) \cdot t^{h-\delta-1+\int_{\beta} c_{1}(S)} \\
& =n_{\delta}(L) \cdot t^{h-\delta-1+\int_{\beta} c_{1}(S)} .
\end{aligned}
$$

The MNOP and Gopakumar-Vafa conjectures state that this should equal a linear combination of stable pair invariants by the universal formulae of [PT09, PT10]. Again things simplify in our case to

$$
\sum_{i=0}^{\infty} r_{i, \beta}(\ldots) q^{1-i}(1+q)^{2 i-2}=\sum_{i=1-h}^{\infty} \mathscr{P}_{i, \beta}^{r e d}(\ldots) q^{i} .
$$

These equations can be inverted to define the $r_{i, \beta}$ as linear combinations of the $\mathscr{P}_{i, \beta}^{r e d}$ :

$$
r_{i, \beta}(\ldots)= \begin{cases}0 & i>h, \\
\mathscr{P}_{1-h, \beta}^{r e d}(\ldots) & i=h \\
\mathscr{P}_{1-i, \beta}^{r e d}(\ldots)-\sum_{k=i+1}^{h}\left(\begin{array}{c}
2 k-2 \\
k-i
\end{array}\right) r_{k, \beta}(\ldots) & i<h .\end{cases}
$$

So one way of stating the conjecture is that the $r_{i, \beta}$ defined by (68) agree with the $r_{i, \beta}$ defined by $(65)$.

In this section we will prove this conjecture for the BPS number $r_{h-\delta, \beta}$, thus showing that the Severi degrees are also given by a linear combination of reduced stable pair invariants. This was the motivation behind the paper [KST11].

We recall from [PT10, Proposition B.8] that $P_{i}(S, \beta)$ is the relative Hilbert scheme of $i-1+h$ points on the fibres of the universal curve over $\operatorname{Hilb}_{\beta}(S)$. (We describe this isomorphism in (90) in Appendix 5.2.) Similarly,

$$
P_{i}\left(S, \mathbb{P}^{j}\right)=\operatorname{Hilb}^{i-1+h}\left(\mathcal{C} / \mathbb{P}^{j}\right)
$$

for any linear system $\mathbb{P}^{j} \subset|L|$. And these spaces are smooth for general $\mathbb{P}^{j}[\mathrm{KST} 11$, Section 4].

TheOREM 5.4. Let $L$ be a $(2 \delta+1)$-very ample line bundle with $H^{1}(L)=0$ and $c_{1}(L)=\beta$ satisfying condition (2) (respectively condition (3)).

The reduced stable pair invariants $\mathscr{P}_{n, \beta}^{r e d}(S)$ (respectively $\left.\mathscr{P}_{n, \iota_{*} \beta}^{r e d}(X)\right)$ contain the Severi degrees $n_{\delta}(L)$ of $\delta$-dimensional linear systems in $|L|$. That is, if we define $r_{i, \beta}(\ldots) \in \mathbb{Z}(t)$ by

$$
\sum_{i=0}^{\infty} r_{i, \beta}(\ldots) q^{1-i}(1+q)^{2 i-2}=\sum_{i=1-h}^{\infty} \mathscr{P}_{i, \beta}^{r e d}(\ldots) q^{i}
$$




\section{Curve COUNTING ON SURFACES I: THEORY}

with $(. .$.$) either of the insertions (63,64)$, then

$$
r_{h-\delta, \beta}(\ldots)=n_{\delta}(L) \cdot t^{h-\delta-1+\int_{\beta} c_{1}(S)},
$$

given by a universal degree- $\delta$ polynomial in $\beta^{2}, \int_{\beta} c_{1}(S), c_{1}(S)^{2}$ and $c_{2}(S)$.

Remark 5.5. We use Appendix 5.2 to deal with curve classes satisfying only condition (2). If we work only with classes satisfying (3) then the reduced Gromov-Witten invariants are also defined and Theorem 5.3 then shows that $R_{h-\delta, \beta}(\ldots)$ is equal to the same linear combination of reduced stable pair invariants. Thus the reduced MNOP conjecture is true in this special case.

Remark 5.6. We can use (69) to define virtual Severi degrees $n_{\delta}(L)$ outside of the very ample case. In the sequel [KT14] we show that these virtual numbers are governed by the Göttsche polynomials [Got98] just as in the very ample case [Tze12, KST11]. In fact we prove that reduced stable pair invariants of surfaces can be calculated in terms of topological numbers much more generally.

Proof. The choice of insertions together with Proposition 5.1 ensure the relevant stable pair moduli spaces for $X$ and $S$ are the same. We work in this proof with $S$.

Let $c=\chi(L)-1-\delta$ denote the codimension of $\mathbb{P}^{\delta} \subset|L|$, and let $i$ run between $1-h$ and $1-h+\delta$.

Since $P_{i}\left(S, \mathbb{P}^{\delta}\right) \subset P_{i}:=P_{i}(S, \beta)$ is smooth of the right reduced virtual dimension (43) $v-$ $h^{0,1}(S)-c=i-(1-h)+\delta$, the reduced obstruction space of Proposition 3.4 (restricted to $\left.P_{i}\left(S, \mathbb{P}^{\delta}\right)\right)$ vanishes. Thus the ordinary obstruction sheaf (restricted to $P_{i}\left(S, \mathbb{P}^{\delta}\right)$ ) is the constant bundle with fibre $H^{2}\left(\mathcal{O}_{S}\right)$, and the fixed obstruction theory $(36)$ of $P_{i}$ (restricted to $P_{i}\left(S, \mathbb{P}^{\delta}\right)$ ) has

$$
h^{0}\left(\left(\left.E_{X}^{\bullet}\right|_{P_{S}}\right)^{f i x}\right)=\Omega_{P_{i}}, \quad h^{-1}\left(\left(\left.E_{X}^{\bullet}\right|_{P_{S}}\right)^{f i x}\right)=H^{0}\left(K_{S}\right) .
$$

Therefore by (37) the virtual normal bundle (restricted to $P_{i}\left(S, \mathbb{P}^{\delta}\right)$ ) similarly has

$$
h^{1}\left(N^{v i r}\right)=\Omega_{P_{i}} \otimes \mathfrak{t}, \quad h^{0}\left(N^{v i r}\right)=H^{0}\left(K_{S}\right) \otimes \mathfrak{t},
$$

where $\mathfrak{t}$ is the one-dimensional representation of $T$ of weight one.

Substituting into the virtual localisation formula (45) and using the insertion formula (57) (and smoothness), we find

$$
\begin{aligned}
\mathscr{P}_{i, \beta}^{r e d}\left(S,\left[\gamma_{1}\right] \ldots\left[\gamma_{b_{1}(S)}\right][p t]^{c}\right) & =\int_{P_{i}\left(S, \mathbb{P}^{\delta}\right)} \frac{c_{t o p}\left(\Omega_{P_{i}} \otimes \mathfrak{t}\right)}{c_{t o p}\left(H^{0}\left(K_{S}\right) \otimes \mathfrak{t}\right)} \\
& =\left(\int_{P_{i}\left(S, \mathbb{P}^{\delta}\right)} c_{\operatorname{dim} P_{i}\left(S, \mathbb{P}^{\delta}\right)}\left(\Omega_{P_{i}}\right)\right) t^{\alpha} .
\end{aligned}
$$

Here $\alpha=\left(\operatorname{rank} \Omega_{P_{i}}-\operatorname{dim} P_{i}\left(S, \mathbb{P}^{\delta}\right)\right)-h^{2,0}(S)$, the first term coming from the numerator, the second from the denominator. This is

$$
\alpha=c+h^{0,1}(S)-h^{2,0}(S)=\chi(L)-\chi\left(\mathcal{O}_{S}\right)-\delta=h-1+\int_{\beta} c_{1}(S)-\delta,
$$


as required. So we concentrate on the bracketed integral in (70). Consider the exact sequence $0 \rightarrow N^{*} \rightarrow \Omega_{P_{i}} \rightarrow \Omega_{P_{i}\left(S, \mathbb{P}^{\delta}\right)} \rightarrow 0$ on $P_{i}\left(S, \mathbb{P}^{\delta}\right)$, where $N^{*}$ is the conormal bundle of $\mathbb{P}^{\delta} \subset \operatorname{Hilb}_{\beta}(S)$, which in turn sits inside an exact sequence $\left.0 \rightarrow \Omega_{\operatorname{Pic}_{\beta}(S)}\right|_{\{L\}} \rightarrow N^{*} \rightarrow \mathcal{O}(-H)^{\oplus c} \rightarrow 0$. We obtain

$$
\begin{aligned}
\left.\int_{P_{i}(S, \mathbb{P} \delta}\right) & c_{\bullet}\left(\Omega_{P_{i}\left(S, \mathbb{P}^{\delta}\right)}\right)\left(1-c H+\frac{c(c-1)}{2} H^{2}-\ldots\right) \\
& =(-1)^{i+h-1+\delta} e\left(P_{i}\left(S, \mathbb{P}^{\delta}\right)\right)+\sum_{j=1}^{\delta}(-1)^{j}\left(\begin{array}{c}
c \\
j
\end{array}\right) \int_{P_{i}\left(S, \mathbb{P}^{\delta}-j\right)} c_{\bullet}\left(\Omega_{P_{i}\left(S, \mathbb{P}^{\delta}\right)}\right) .
\end{aligned}
$$

Here the $\mathbb{P}^{\delta-j}$ s are generic linear subspaces of $\mathbb{P}^{\delta} \subset|L|$ chosen to contain only curves of geometric genus at least $h-\delta+j$ (see Proposition 5.1) and so that the $P_{i}\left(S, \mathbb{P}^{\delta-j}\right)$ are still smooth.

Similarly, using the exact sequence $0 \rightarrow \mathcal{O}(-H)^{\oplus j} \rightarrow \Omega_{P_{i}\left(S, \mathbb{P}^{\delta}\right)} \rightarrow \Omega_{P_{i}\left(S, \mathbb{P}^{\delta}-j\right)} \rightarrow 0$ on $P_{i}\left(S, \mathbb{P}^{\delta-j}\right)$, we find inductively that the whole expression can be written in terms of topological Euler characteristics

$$
(-1)^{i+h-1+\delta}\left(e\left(P_{i}\left(S, \mathbb{P}^{\delta}\right)\right)+\sum_{j=1}^{\delta} a_{j} e\left(P_{i}\left(S, \mathbb{P}^{\delta-j}\right)\right)\right)
$$

for some integral coefficients $a_{j}$.

Therefore the $r_{i, \beta} \in \mathbb{Z}(t)$ are defined by setting $\sum_{i=0}^{\infty} r_{i, \beta} q^{1-i}(1+q)^{2 i-2}$ equal to $t^{h-1+\int_{\beta} c_{1}(S)-\delta}$ times

$$
\sum_{i=1-h}^{1-h+\delta}(-1)^{i+h-1+\delta}\left(e\left(P_{i}\left(S, \mathbb{P}^{\delta}\right)\right)+\sum_{j=1}^{\delta} a_{j} e\left(P_{i}\left(S, \mathbb{P}^{\delta-j}\right)\right)\right) q^{i}+O\left(q^{2-h+\delta}\right) .
$$

Evaluating these Euler characteristics fibrewise, we express them as Euler characteristics of the base $\mathbb{P}^{\delta-j} \mathrm{~s}$, weighted by the constructible function whose value at a point is the Euler characteristic of the fibre above it. Then by inverting the above formula as in (68), we also get each $r_{i, \beta}$ as a sum of weighted Euler characteristics of the $\mathbb{P}^{\delta-j} \mathrm{~s}$.

By [PT10, Theorem 5], the weighting function for $r_{i, \beta}$ at a point $C \in \mathbb{P}^{\delta-j}$ is zero unless $i$ lies between the arithmetic and geometric genera of the reduced irreducible curve $C$. In particular, for $i=h-\delta$, the function is identically zero on $\mathbb{P}^{\delta-j}$ for $j>0$, and nonzero on $\mathbb{P}^{\delta}$ only at the $\delta$-nodal curves $D_{i}$. And by [PT10, Proposition 3.23], it takes the value 1 on the $D_{i}$.

So in (71) only the first terms contribute to $r_{h-\delta, \beta}$; the second terms all cancel to give zero. And the first Euler characteristic contributes the number of $\delta$-nodal curves $D_{i}$, yielding

$$
r_{h-\delta, \beta}(\ldots)=n_{\delta}(L) \cdot t^{h-1+\int_{\beta} c_{1}(S)-\delta}
$$

as claimed. 


\section{Curve COUNTING ON SURFACES I: THEORY}

\section{ACKNOWLEDGEMENTS}

This project originated with a suggestion of Daniel Huybrechts, for which we are very grateful. We would like to thank Nicolas Addington, Jim Bryan, Davesh Maulik, Vivek Shende, an excellent referee and particularly Rahul Pandharipande for their assistance. We owe an obvious intellectual debt to the papers [BL00, MPT10].

\section{Appendix A. Reduced obstruction theory for stable pairs revisited written with Dmitri Panov}

\section{A.1 The Hilbert scheme of curves as a zero locus ${ }^{6}$}

Let $H_{\beta}:=\operatorname{Hilb}_{\beta}(S)$ denote the Hilbert scheme of curves in class $\beta$. We describe an embedding of $H_{\beta}$ in a smooth space with a bundle over it and a canonical section whose zero locus is precisely $H_{\beta}$. For now we make an assumption slightly stronger than condition (2):

$$
H^{2}(L)=0 \quad \text { for all line bundles with } c_{1}(L)=\beta .
$$

Fix a curve $A \subset S$ which is sufficiently ample in the sense that

$$
H^{\geqslant 1}(L(A))=0 \quad \text { for all line bundles with } c_{1}(L)=\beta .
$$

Notice that the constructions of this section can also be done in projective families of surfaces by picking a divisor $A$ on the whole family which has the required ampleness on each fibre. Letting $\gamma=[A]+\beta$, we get an embedding $H_{\beta} \hookrightarrow H_{\gamma}$ defined on points by

$$
\begin{aligned}
\operatorname{Hilb}_{\beta}(S) & \hookrightarrow \operatorname{Hilb}_{\gamma}(S), \\
C & \mapsto A+C .
\end{aligned}
$$

At the level of schemes, the map is defined using the usual universal diagram

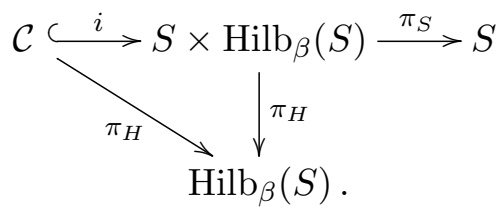

The divisor $\mathcal{C}+\pi_{S}^{*} A \subset S \times H_{\beta}$ is a flat family of divisors in $S$ of class $\gamma$, so has a classifying map from the base $\operatorname{Hilb}_{\beta}(S)$ to $\operatorname{Hilb}_{\gamma}(S)$.

Notice that by (73), $H_{\gamma}$ is smooth; it is a projective bundle over $\operatorname{Pic}_{\gamma}(S)$. Let $\mathcal{D} \subset S \times H_{\gamma}$ be the universal divisor, and again use $\pi_{H}$ to denote the projection to $H_{\gamma}$. Consider $\pi_{H *}$ applied to the exact sequence

$$
\left.0 \longrightarrow \mathcal{O}\left(\mathcal{D}-\pi_{S}^{*} A\right) \longrightarrow \mathcal{O}(\mathcal{D}) \longrightarrow \mathcal{O}(\mathcal{D})\right|_{\pi_{S}^{*} A} \longrightarrow 0
$$

${ }^{6}$ We now realise this construction was discovered many years ago by Dürr, Kabanov and Okonek [DKO07]. In the next Section A.2 we extend it to stable pairs. 
By our assumption (72), $R^{2} \pi_{H *}$ of the first term vanishes, and $R^{\geqslant 1} \pi_{H *}$ of the central term vanishes by (73). Therefore $R^{\geqslant 1} \pi_{H *}$ of the final term vanishes, and

$$
F:=\pi_{H *}\left(\left.\mathcal{O}(\mathcal{D})\right|_{\pi_{S}^{*} A}\right)
$$

is a vector bundle on $H_{\gamma}$.

Proposition A.1. Define the canonical section $\sigma$ of $F \rightarrow \operatorname{Hilb}_{\gamma}(S)$ by pushing down the restriction $\left.s_{\mathcal{D}}\right|_{\pi_{S}^{*} A}$ of the canonical section of $\mathcal{O}(\mathcal{D})$. Then the zero locus of $\sigma$ is the subscheme

$$
A+\operatorname{Hilb}_{\beta}(S) \subset \operatorname{Hilb}_{\gamma}(S) .
$$

Proof. By its very definition, $\sigma(D)=0$ for $D \in \operatorname{Hilb}_{\gamma}(S)$ if and only if $\left.s_{D}\right|_{A}=0$, if and only if $A \subset D$. This gives the result at the level of sets. In fact, scheme-theoretically, it is also clear that $\sigma$ vanishes on $A+\operatorname{Hilb}_{\beta}(S)$. So it is sufficient to produce the inverse morphism $Z(\sigma) \rightarrow A+\operatorname{Hilb}_{\beta}(S)$.

Since $s_{\mathcal{D}}$ vanishes on the pullback of $A$ to $S \times Z(\sigma)$, we can divide by the pullback of its defining equation $s_{A}$ to give a divisor in $S \times Z(\sigma)$ whose classifying map $Z(\sigma) \rightarrow \operatorname{Hilb}_{\beta}(S)$ gives the required inverse.

Letting $I$ denote the ideal of $H_{\beta} \subset H_{\gamma}$, we get the diagram on $Z(\sigma)=H_{\beta}$ :

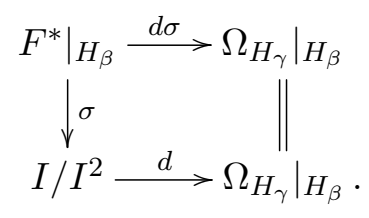

We denote the upper row by the complex $\left\{F^{-1} \rightarrow F^{0}\right\}=: F_{\text {red }}^{\bullet}$ of vector bundles on $H_{\beta}$. The bottom row is the truncated cotangent complex of $H_{\beta}$, so we have

$$
F_{r e d}^{\bullet} \rightarrow \mathbb{L}_{\operatorname{Hilb}_{\beta}(S)} \text {. }
$$

This is an isomorphism on $h^{0}=\Omega_{H_{\beta}}$ and a surjection on $h^{-1}$ because $\left.F^{*}\right|_{H_{\beta}} \rightarrow I / I^{2}$ is onto.

We will see that $F_{r e d}^{\bullet}$ is the reduced obstruction theory. As a warm-up we explain what happens at a point $C \in \operatorname{Hilb}_{\beta}(S)$. The obvious long exact sequence

$$
\begin{aligned}
0 \rightarrow H^{0}\left(\mathcal{O}_{C}(C)\right) \rightarrow & H^{0}\left(\mathcal{O}_{A+C}(A+C)\right) \rightarrow H^{0}\left(\mathcal{O}_{A}(A+C)\right) \rightarrow \\
& H^{1}\left(\mathcal{O}_{C}(C)\right) \rightarrow H^{1}\left(\mathcal{O}_{A+C}(A+C)\right) \rightarrow H^{1}\left(\mathcal{O}_{A}(A+C)\right)
\end{aligned}
$$

is precisely

$$
\left.0 \rightarrow T_{C} H_{\beta} \rightarrow T_{C+A} H_{\gamma} \rightarrow F\right|_{\{C+A\}} \rightarrow H^{1}\left(\mathcal{O}_{C}(C)\right) \rightarrow H^{2}\left(\mathcal{O}_{S}\right) \rightarrow 0,
$$

using the assumptions $(72,73)$ and identifying the penultimate term via the exact sequence

$$
0 \rightarrow \mathcal{O}_{S} \rightarrow \mathcal{O}(A+C) \rightarrow \mathcal{O}_{A+C}(A+C) \rightarrow 0 .
$$

In other words, we identify $H^{1}\left(\mathcal{O}_{A+C}(A+C)\right) \stackrel{\sim}{\longrightarrow} H^{2}\left(\mathcal{O}_{S}\right)$ by the semi-regularity map for $A+C$. But the resulting map $H^{1}\left(\mathcal{O}_{C}(C)\right) \rightarrow H^{2}\left(\mathcal{O}_{S}\right)$ in $(78)$ is the semi-regularity map for $C$ 


\section{Curve COUNTING ON SURFACES I: THEORY}

itself, as can be seen from the comparison maps between the sequence $0 \rightarrow \mathcal{O}_{S} \rightarrow \mathcal{O}(A+C) \rightarrow$ $\mathcal{O}_{A+C}(A+C) \rightarrow 0$ and the sequence of subsheaves $0 \rightarrow \mathcal{O}_{S} \rightarrow \mathcal{O}(C) \rightarrow \mathcal{O}_{C}(C) \rightarrow 0$.

The first few maps in (78) are, respectively, the derivative at $C$ of the inclusion $H_{\beta} \hookrightarrow H_{\gamma}$, and the derivative at $C+A$ of the section $s \in \Gamma(F)$. This latter map is exactly the complex $\left.\left(F_{r e d}^{\bullet}\right)^{\vee}\right|_{C}$ (that is, the dual of the top line of $\left.(77)\right)$, with cokernel

$$
h^{1}\left(\left(F_{r e d}^{\bullet}\right)^{\vee}\right)=\operatorname{ker}\left(H^{1}\left(\mathcal{O}_{C}(C)\right) \rightarrow H^{2}\left(\mathcal{O}_{S}\right)\right) .
$$

Thus the obstruction space of $F_{r e d}^{\bullet}$ at $C$ is the reduced obstruction space: the kernel of the semi-regularity map of (1) from the usual obstruction space $H^{1}\left(\mathcal{O}_{C}(C)\right)$ to $H^{2}\left(\mathcal{O}_{S}\right)$.

We now prove all this for the universal curve $\mathcal{C}$ over the family $\operatorname{Hilb}_{\beta}(S)$. The ordinary obstruction theory $F^{\bullet} \rightarrow \mathbb{L}_{H_{\beta}}$, where $\left(F^{\bullet}\right)^{\vee}:=R \pi_{H *} \mathcal{O}_{\mathcal{C}}(\mathcal{C})$, admits the semi-regularity map

$$
\left(F^{\bullet}\right)^{\vee}=R \pi_{H *} \mathcal{O}_{\mathcal{C}}(\mathcal{C}) \longrightarrow R^{2} \pi_{H *} \mathcal{O}_{S \times H_{\beta}}[-1]
$$

induced by the exact sequence $0 \rightarrow \mathcal{O} \rightarrow \mathcal{O}(\mathcal{C}) \rightarrow \mathcal{O}_{\mathcal{C}}(\mathcal{C}) \rightarrow 0$.

Proposition A.2. The semi-regularity map fits into an exact triangle intertwining the two perfect obstruction theory maps:

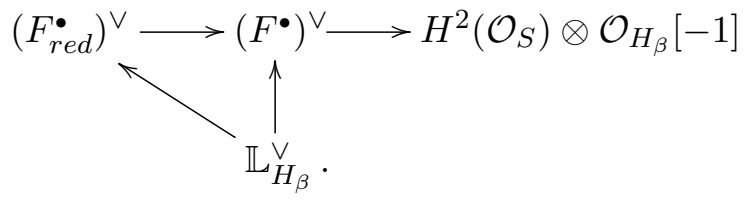

Proof. First we recall the obstruction theory of $H_{\beta}$. Using the diagram (75) we get maps

$$
\mathcal{O}_{\mathcal{C}}(-\mathcal{C})=\mathbb{L}_{\mathcal{C} /\left(S \times H_{\beta}\right)}[-1] \longrightarrow L i^{*} \mathbb{L}_{S \times H_{\beta}}=\pi_{S}^{*} \mathbb{L}_{S} \oplus \pi_{H}^{*} \mathbb{L}_{H_{\beta}} \longrightarrow \pi_{H}^{*} \mathbb{L}_{H_{\beta}}
$$

Their composition gives, by adjunction, the perfect obstruction theory ${ }^{7}$

$$
\mathbb{L}_{H_{\beta}}^{\vee} \longrightarrow R \pi_{H *} \mathcal{O}_{\mathcal{C}}(\mathcal{C})
$$

Similar working using $\mathcal{O}_{\mathcal{D}}(-\mathcal{D})$ on $S \times H_{\gamma}$ gives (recalling that $H_{\gamma}$ is smooth),

$$
T_{H_{\gamma}}=\mathbb{L}_{H_{\gamma}}^{\vee} \longrightarrow R \pi_{H *} \mathcal{O}_{\mathcal{D}}(\mathcal{D}) \text {. }
$$

On restriction to $H_{\beta} \stackrel{+A}{\longrightarrow} H_{\gamma}$, the divisor $\mathcal{D}$ pulls back to $\pi_{S}^{*} A+\mathcal{C}$, so we have the exact sequence

$$
0 \longrightarrow \mathcal{O}_{\mathcal{C}}(\mathcal{C}) \longrightarrow \mathcal{O}_{\mathcal{D}}(\mathcal{D}) \longrightarrow \mathcal{O}_{\pi_{S}^{*} A}(\mathcal{D}) \longrightarrow 0
$$

\footnotetext{
${ }^{7}$ This construction, viewing curves as divisors in $S$, coincides with the obstruction theory obtained by thinking of $\operatorname{Hilb}_{\beta}(S)$ as parameterising pairs $\left(\mathcal{O}_{C}, 1\right)$ of a sheaf and a section. The essential point is that the Atiyah class of $\mathcal{O}_{\mathcal{C}}$ in $\operatorname{Ext}^{1}\left(\mathcal{O}_{\mathcal{C}}, \mathcal{O}_{\mathcal{C}} \otimes \mathbb{L}_{S \times H_{\beta}}\right)$ is the canonical morphism in the summand $\operatorname{Hom}\left(\mathcal{O}_{\mathcal{C}}(-\mathcal{C}), L i^{*} \mathbb{L}_{S \times H_{\beta}}\right)$.
} 
over $H_{\beta}$. Pushing down to $H_{\beta}$ and combining with the maps $(81,82)$ gives the commutative diagram

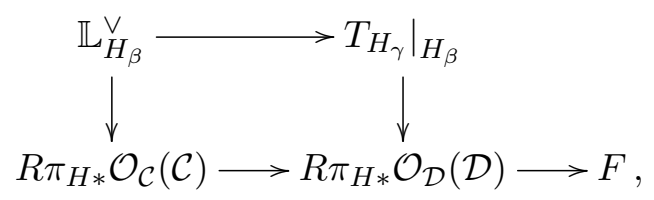

where the bottom row is an exact triangle and the composition $\left.T_{H_{\gamma}}\right|_{H_{\beta}} \rightarrow F$ is $d \sigma$. Thus we get the diagram

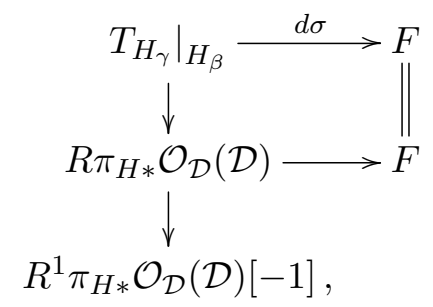

whose columns are exact triangles since $T_{H_{\gamma}} \stackrel{\sim}{\longrightarrow} \pi_{H *} \mathcal{O}_{\mathcal{D}}(\mathcal{D})$. The cone on the first row is $\left(F_{\text {red }}^{\bullet}\right)^{\vee}$ (77), the second is $\left(F^{\bullet}\right)^{\vee}$ by $(83)$, and the third is $H^{2}\left(\mathcal{O}_{S}\right) \otimes \mathcal{O}_{H_{\beta}}[-1]$ by the exact sequence $0 \rightarrow \mathcal{O}_{S \times H_{\beta}} \rightarrow \mathcal{O}_{S \times H_{\beta}}(\mathcal{D}) \rightarrow \mathcal{O}_{\mathcal{D}}(\mathcal{D}) \rightarrow 0$. We get the exact triangle

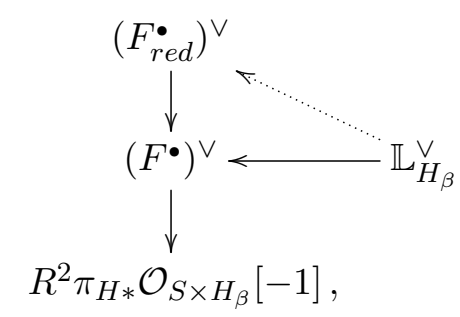

where we have added in the map (81). Though the bottom arrow was constructed from the semi-regularity map for $\mathcal{D}$, it is the semi-regularity map $\left(F^{\bullet}\right)^{\vee} \rightarrow H^{2}\left(\mathcal{O}_{S}\right) \otimes \mathcal{O}_{H_{\beta}}[-1]$ for $\mathcal{C}$ by the commutativity of the diagram of exact triangles

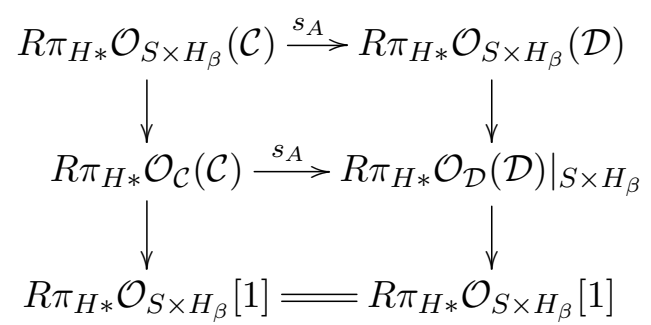

and the functoriality of truncation.

Finally then, we need the dotted arrow in (85). Considering the map $\mathbb{L}_{H_{\beta}}^{\vee} \rightarrow\left(F^{\bullet}\right)^{\vee}$ of (81) as a morphism to the cone on the second row of the diagram (84), the diagram (83) shows that it factors through the complex $\left\{\left.T_{H_{\gamma}}\right|_{H_{\beta}} \rightarrow F\right\}$, which is $\left(F_{r e d}^{\bullet}\right)^{\vee}$, as required. 


\section{CURVE COUNTING ON SURFACES I: THEORY}

\section{A.2 The relative Hilbert scheme of points as a zero locus}

Recall that the moduli space of stable pairs is a relative Hilbert scheme of points over $H_{\beta}$ :

$$
P_{1-h+n}(S, \beta) \cong \operatorname{Hilb}^{n}\left(\mathcal{C} / H_{\beta}\right) .
$$

Just as we described the base $H_{\beta}$ as the zero locus of a section of a bundle, we can do the same for the fibres. We use the embedding

$$
\operatorname{Hilb}^{n}\left(\mathcal{C} / H_{\beta}\right) \subset S^{[n]} \times H_{\beta},
$$

where $S^{[n]}:=\operatorname{Hilb}^{n}(S)$ is smooth because $S$ is a surface. There is a universal subscheme

$$
\mathcal{Z} \subset S \times S^{[n]} \times H_{\beta} \stackrel{\pi}{\longrightarrow} S^{[n]} \times H_{\beta}
$$

pulled back from $S \times S^{[n]}$, and of course a universal curve $\mathcal{C}$ pulled back from $S \times H_{\beta}$ inducing a universal line bundle and section $\left(\mathcal{O}(\mathcal{C}), s_{\mathcal{C}}\right)$. This induces a canonical section $\sigma_{\mathcal{C}}$ of the rank $n$ vector bundle

$$
\mathcal{O}(\mathcal{C})^{[n]}:=\pi_{*}\left(\left.\mathcal{O}(\mathcal{C})\right|_{\mathcal{Z}}\right)
$$

Its zeros are the pairs $(Z, C)$ with $Z \subset C \subset S$; in fact by the argument of Proposition A.1 it is scheme-theoretically $\operatorname{Hilb}^{n}\left(\mathcal{C} / H_{\beta}\right)$ embedded as in (86).

Thus we get a perfect relative obstruction theory in the usual way:

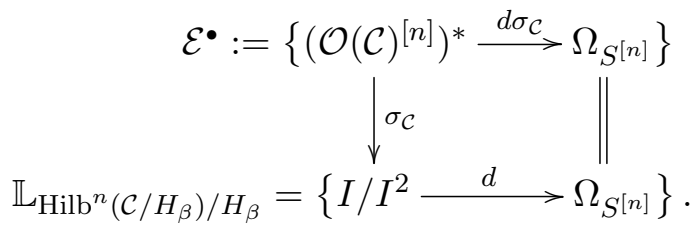

\section{A.3 Identifying the obstruction theories}

Let $P$ denote $P_{1-h+n}(S, \beta) \cong \operatorname{Hilb}^{n}\left(\mathcal{C} / H_{\beta}\right)$. We now have a perfect relative obstruction theory $\mathcal{E}^{\bullet}$ for $P / H_{\beta}$ by (88), and ordinary and reduced perfect obstruction theories $F^{\bullet}, F_{\text {red }}^{\bullet}$ for $H_{\beta}$ (Proposition A.2) when condition (2) holds.

We would like to combine them to give ordinary and perfect absolute obstruction theories for $P$ under condition (2), and to know that they coincide with the ordinary and reduced perfect obstruction theories $E^{\bullet}, E_{r e d}^{\bullet}$ of Proposition 3.4 when condition (3) is also satisfied.

That is, we want commutative diagrams of exact triangles:
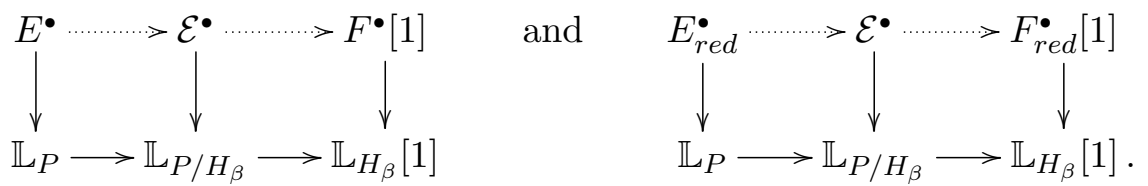

We will first define the upper exact triangles, then prove exactness of the lower rows, and finally turn to commutativity of the resulting squares. 


\section{Kool, R. P. Thomas}

For ease of exposition, in this section we will work at one point $(F, s)$ of $P$ at a time. As in the rest of the paper, all of the arguments extend over the whole family $P$ in the usual way, modulo more cumbersome notation.

Proposition A.3. The obstruction theory $E^{\bullet}=\left(R \operatorname{Hom}\left(I^{\bullet}, F\right)\right)^{\vee}$ of Proposition 3.4 sits in a natural exact triangle with the relative obstruction theory $\mathcal{E}^{\bullet}$ of $P / H_{\beta}$ (88) and the obstruction theory $F^{\bullet}(79)$ for $H_{\beta}$ :

$$
F^{\bullet} \longrightarrow E^{\bullet} \longrightarrow \mathcal{E}^{\bullet}
$$

To prove this we need to recall from [PT10, Appendix B] the construction of the isomorphism between the moduli space of stable pairs and the relative Hilbert scheme of points on the fibres of $\mathcal{C}$,

$$
P_{1-h+n}(S, \beta) \cong \operatorname{Hilb}^{n}\left(\mathcal{C} / H_{\beta}\right) .
$$

Given a pair $(F, s)$ with scheme-theoretic support $C \in H_{\beta}$, we dualise

$$
0 \longrightarrow \mathcal{O}_{C} \longrightarrow F \longrightarrow Q \longrightarrow 0
$$

considered as sheaves and maps of sheaves on $C$. That is, applying $(\cdot)^{*}:=\mathscr{H}_{0} m_{C}\left(\cdot, \mathcal{O}_{C}\right)$ gives

$$
0 \longrightarrow F^{*} \longrightarrow \mathcal{O}_{C} \longrightarrow \mathscr{E} x t_{C}^{1}\left(Q, \mathcal{O}_{C}\right) \longrightarrow 0
$$

with all higher Ext sheaves zero [PT10, Proposition B.5]. Therefore $F^{*}$ is an ideal sheaf on $C$; denoting the corresponding subscheme by $Z \subset C$ we can write the above sequence as

$$
0 \longrightarrow I_{Z \subset C} \longrightarrow \mathcal{O}_{C} \longrightarrow \mathcal{O}_{Z} \longrightarrow 0 \text {. }
$$

Then $Z \in \operatorname{Hilb}^{n} C$ defines our point $(Z, C) \in \operatorname{Hilb}^{n}\left(\mathcal{C} / H_{\beta}\right)$.

Lemma A.4. The derived dual of $i_{*} F$ is $i_{*}\left(I_{Z \subset C}\right)(C)[-1]$. The derived dual of the complex $I^{\bullet}:=\left\{\mathcal{O}_{S} \stackrel{s}{\longrightarrow} i_{*} F\right\}$ is the ideal sheaf of $Z \subset S$ twisted by the line bundle $\mathcal{O}_{S}(C)$ :

$$
\left(I^{\bullet}\right)^{\vee} \cong \mathscr{I}_{Z}(C) \text {. }
$$

Proof. Since $C$ is a Cartier divisor in $S$, it is Gorenstein with canonical bundle $\left.K_{S}(C)\right|_{C}$. Therefore Serre duality for the inclusion $i: C \hookrightarrow S$ gives

$$
\begin{aligned}
\left(i_{*} F\right)^{\vee}=\operatorname{RHom}\left(i_{*} F, \mathcal{O}_{S}\right) \cong i_{*} R \mathscr{H} o m & \left(F, i^{!} \mathcal{O}_{S}\right) \cong i_{*} R \mathscr{H o m}\left(F, \mathcal{O}_{C}(C)[-1]\right) \\
& \cong i_{*}\left(F^{*}\right)(C)[-1] \cong i_{*}\left(I_{Z \subset C}\right)(C)[-1] .
\end{aligned}
$$

Dualising the commutative diagram of exact triangles

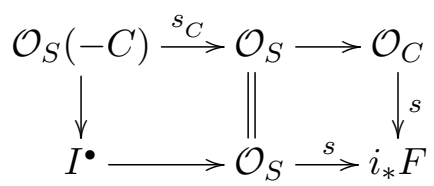


yields

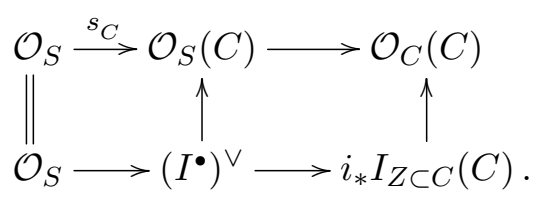

The top row is the obvious exact sequence defined by the canonical extension class in $\operatorname{Ext}^{1}\left(\mathcal{O}_{C}(C), \mathcal{O}_{S}\right)$. Pulling this back via the right-hand arrow $i_{*} I_{Z \subset C}(C) \rightarrow \mathcal{O}_{C}(C)$ gives the extension class in $\operatorname{Ext}^{1}\left(i_{*} I_{Z \subset C}, \mathcal{O}_{S}\right)$ of the bottom row. Therefore $\left(I^{\bullet}\right)^{\vee}$ is the kernel of the induced map from $\mathcal{O}_{S}(C)$ to the cokernel of the right-hand arrow. Since this arrow is the canonical inclusion, it has quotient $\mathcal{O}_{Z}(C)$ and $\left(I^{\bullet}\right)^{\vee}$ is $\mathscr{I}_{Z}(C)$ as claimed.

Proof of Proposition A.3. By Lemma A.4, the ordinary deformation-obstruction complex $\left(E^{\bullet}\right)^{\vee}$ at $(F, s) \in P$ can be written

$$
\left(E^{\bullet}\right)^{\vee}:=R \operatorname{Hom}\left(I^{\bullet}, F\right)=R \operatorname{Hom}\left(F^{\vee},\left(I^{\bullet}\right)^{\vee}\right)=R \operatorname{Hom}_{S}\left(i_{*} I_{Z \subset C}, \mathscr{I}_{Z}\right)[1]
$$

at $(Z \subset C) \in P$.

Via the obvious exact sequences we get a commuting diagram of exact triangles (in which some $i_{*} \mathrm{~s}$ are suppressed, and all $R \mathscr{H}$ oms are taken on $S$ ),

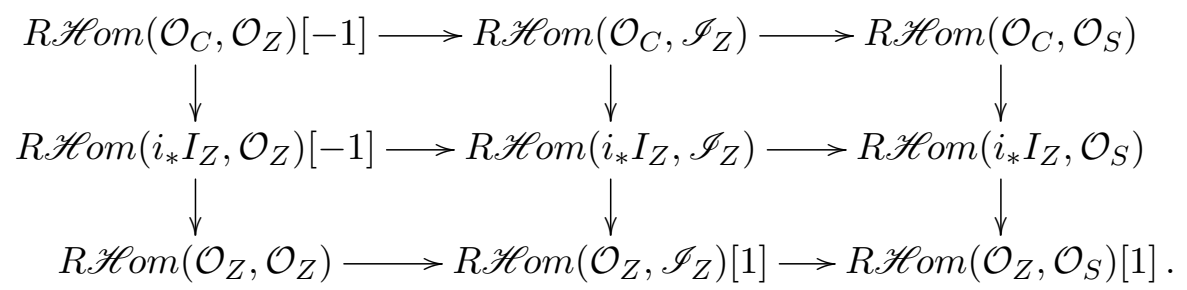

The top-degree cohomologies of the terms in the bottom right and bottom left corners are the same via the induced homomorphism between them:

$$
\mathscr{E} x t^{2}\left(\mathcal{O}_{Z}, \mathcal{O}_{S}\right) \stackrel{\sim}{\longrightarrow} \mathscr{E} x t^{2}\left(\mathcal{O}_{Z}, \mathcal{O}_{Z}\right)
$$

(For instance, this may be seen by using Serre duality and the fact that the obvious map $\mathscr{H} o m\left(\mathcal{O}_{Z}, \mathcal{O}_{Z}\right) \rightarrow \mathscr{H} o m\left(\mathcal{O}_{S}, \mathcal{O}_{Z}\right)$ is an isomorphism.) The same goes for the lowest degree cohomologies of the bottom left and top left terms:

$$
\mathscr{H} \text { om }\left(\mathcal{O}_{Z}, \mathcal{O}_{Z}\right) \stackrel{\sim}{\longrightarrow} \mathscr{H} \text { om }\left(\mathcal{O}_{C}, \mathcal{O}_{Z}\right)
$$

Thus, in the central horizontal exact triangle, we can take the cone on the map to (93), then the cone on the map from (94), to yield a new exact triangle

$$
\left\{\begin{array}{c}
\mathscr{E} x t^{1}\left(\mathcal{O}_{Z}, \mathcal{O}_{Z}\right) \\
\downarrow \\
\mathscr{E} x t^{1}\left(\mathcal{O}_{C}, \mathcal{O}_{Z}\right)
\end{array}\right\} \longrightarrow R \mathscr{H} \circ \mathrm{om}\left(i_{*} I_{Z}, \mathscr{I}_{Z}\right)[1] \longrightarrow R \mathscr{H} o m\left(\mathcal{O}_{C}, \mathcal{O}_{S}\right)[1]
$$


The vertical arrow is constructed from the restriction map $\mathcal{O}_{C} \rightarrow \mathcal{O}_{Z}$. Identifying $\mathscr{E} x t^{1}\left(\mathcal{O}_{C}, \mathcal{O}_{Z}\right)$ with $O_{Z}(C)$ using $s_{C}$, it becomes the derivative of $s_{C}$. Similarly, we can identify the last term $R \mathscr{H}$ om $\left(\mathcal{O}_{C}, \mathcal{O}_{S}\right)$ with $\mathcal{O}_{C}(C)[-1]$. On applying $R \Gamma$ (or, in the family case, pushing down to the moduli space $P$ ) we get the exact triangle

$$
\left\{\begin{array}{c}
T_{Z} S^{[n]} \\
\downarrow \\
\mathcal{O}(C)^{[n]}
\end{array}\right\} \longrightarrow R \operatorname{Hom}\left(i_{*} I_{Z}, \mathscr{I}_{Z}\right)[1] \longrightarrow R \Gamma\left(\mathcal{O}_{C}(C)\right)
$$

with first term $\left(\mathcal{E}^{\bullet}\right)^{\vee}$ of $(88)$. By (91) we have the required exact triangle

$$
\left(\mathcal{E}^{\bullet}\right)^{\vee} \longrightarrow\left(E^{\bullet}\right)^{\vee} \longrightarrow\left(F^{\bullet}\right)^{\vee} \text {. }
$$

Since we have been working with truncated, rather than full, cotangent complexes, we have to be careful about exactness.

Lemma A.5. The canonical maps of truncated cotangent complexes

$$
\mathbb{L}_{H_{\beta}} \longrightarrow \mathbb{L}_{P} \longrightarrow \mathbb{L}_{P / H_{\beta}}
$$

define an exact triangle.

Proof. We need to show that the following triangle of complexes

$$
\left\{I /\left.\left.I^{2}\right|_{P} \rightarrow \Omega_{H_{\gamma}}\right|_{P}\right\} \longrightarrow\left\{\mathscr{I} /\left.\mathscr{I}^{2} \rightarrow\left(\Omega_{S^{[n]}} \oplus \Omega_{H_{\gamma}}\right)\right|_{P}\right\} \longrightarrow\left\{\mathcal{J} /\left.\mathcal{J}^{2} \rightarrow \Omega_{S^{[n]}}\right|_{P}\right\}
$$

is exact. Here the ideal of $H_{\beta} \subset H_{\gamma}$ is denoted by $I$, the ideal of $P \subset S^{[n]} \times H_{\gamma}$ is $\mathscr{I}$, and the ideal of $P \subset S^{[n]} \times H_{\beta}$ is $\mathcal{J}:=\mathscr{I} /(\mathscr{I} \cap I)$. The arrows are the obvious ones, and we have suppressed some (flat) pullbacks. We therefore need to prove the exactness of

$$
0 \rightarrow I /\left(I^{2}+I \cdot \mathscr{I}\right) \rightarrow \mathscr{I} / \mathscr{I}^{2} \rightarrow \mathcal{J} / \mathcal{J}^{2} \rightarrow 0
$$

on the left. That is, since $I^{2}+I \cdot \mathscr{I}=I \cdot \mathscr{I}$, we need to show that

$$
I \cap \mathscr{I}^{2} \subseteq I \cdot \mathscr{I} \text {. }
$$

First work on the open set in $P \cong \operatorname{Hilb}^{n}\left(\mathcal{C} / H_{\beta}\right) \ni(C, Z)$ where the zero-dimensional subscheme $Z$ is disjoint from $A \cap C$. (Recall that $A$ was a fixed ample divisor used to embed $H_{\beta}$ into $H_{\gamma}(74)$.) Then in a neighbourhood in $S^{[n]} \times H_{\gamma}$ of this open set, we have $\mathscr{I}=I+J$, where $J$ is the ideal sheaf of the subscheme

$$
\operatorname{Hilb}^{n}\left(\mathcal{D} / H_{\gamma}\right) \subset S^{[n]} \times H_{\gamma} .
$$

(In other words, the locus of pairs $(Z \subset C)$ is locally isomorphic to the locus of pairs $(Z \subset(A \cup C))$ so long as $Z$ stays away from $A \cap C$.) But the relative Hilbert scheme (96) is flat over $H_{\gamma}$ : as in [AIK77], the fact that each fibre has dimension $n$ follows immediately from the fact that the punctual Hilbert scheme of length $r$ subschemes supported at a single point of a smooth surface has dimension $r-1$ [Iar72], so for a curve in a surface it has dimension at most $(r-1)$. 


\section{Curve COUNTING ON SURFACES I: THEORY}

Then, since each fibre is cut out of the smooth $2 n$-dimensional space $S^{[n]}$ by a section of a rank $n$ vector bundle, this section is regular and the associated Koszul resolution exact. Thus the Hilbert polynomial of each fibre can be calculated in terms of the Chern classes of this bundle on $S$, independently of the fibre. This implies flatness.

Furthermore, $I$ is pulled back from $H_{\gamma}$, so $I \cap J^{2} \subseteq I \cap J=I \cdot J$. Therefore

$$
I \cap \mathscr{I}^{2}=I \cap(I+J)^{2}=I \cap\left(I^{2}+I \cdot J+J^{2}\right)=I^{2}+I \cdot J+I \cap J^{2} \subseteq I^{2}+I \cdot J=I \cdot \mathscr{I},
$$

giving (95).

This gives exactness over an open set of $P$. By changing the divisor $A$ we can cover $P$ with open sets on which we get exactness. Since exactness can be checked locally, we are done.

Combining the map $\mathcal{E}^{\bullet} \rightarrow F^{\bullet}[1]$ of Proposition A.3 with the maps (88) and (81), we get the square

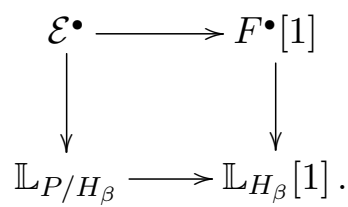

Lemma A.6. The square (97) commutes.

Proof. We use the following general fact. Suppose that a scheme $P / B$ over a (possibly singular) base $B$ is cut out of the product $A \times B$ by a section $\sigma$ of a vector bundle $E$. If we further assume that $A$ is smooth then we get a natural induced relative perfect obstruction theory

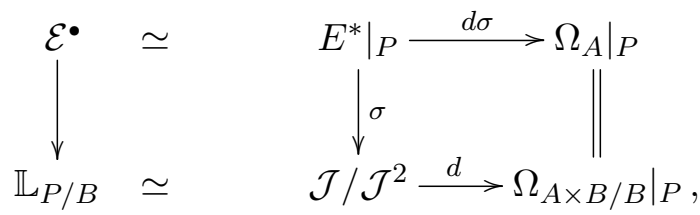

where $\mathcal{J} \subset \mathcal{O}_{A \times B}$ is the ideal sheaf of $P$.

Then the composition of the maps $\mathcal{E}^{\bullet} \rightarrow \mathbb{L}_{P / B} \rightarrow \mathbb{L}_{B}[1]$ factors through the obvious map $\left.\mathcal{E}^{\bullet} \rightarrow E^{*}\right|_{P}[1]$ via the map

$$
\left.E^{*}\right|_{P} \longrightarrow \mathbb{L}_{B}
$$

which is the composition

$$
\left.E^{*}\right|_{P} \stackrel{\sigma}{\longrightarrow} \mathcal{J} / \mathcal{J}^{2}=\left.\mathbb{L}_{P /(A \times B)}[-1] \longrightarrow \mathbb{L}_{A \times B}\right|_{P} \longrightarrow \mathbb{L}_{B}
$$

Here the last map is just projection onto the second factor of $\mathbb{L}_{A} \oplus \mathbb{L}_{B}$, and as usual we have suppressed some pull back maps.

We use this in the setting of Section A.2, with $A=S^{[n]}, B=H_{\beta}, E=\mathcal{O}(\mathcal{C})^{[n]}$ and $\sigma=\sigma_{\mathcal{C}}$. See (87). Then going anticlockwise round (97) gives the composition

$$
\mathcal{E}^{\bullet} \longrightarrow\left(\mathcal{O}(\mathcal{C})^{[n]}\right)^{*}[1] \stackrel{\sigma_{\mathcal{C}}}{\longrightarrow} \mathcal{J} /\left.\mathcal{J}^{2}[1] \longrightarrow\left(\mathbb{L}_{S^{[n]}} \oplus \mathbb{L}_{H_{\beta}}\right)\right|_{P}[1] \longrightarrow \mathbb{L}_{H_{\beta}}[1]
$$


The second arrow is given by the canonical section $\sigma_{\mathcal{C}}$ of $\mathcal{O}(\mathcal{C})^{[n]}(87)$. This is pushed down from the universal zero-dimensional subscheme $\mathcal{Z}$ inside the universal curve $\mathcal{C}$ over $P$ :

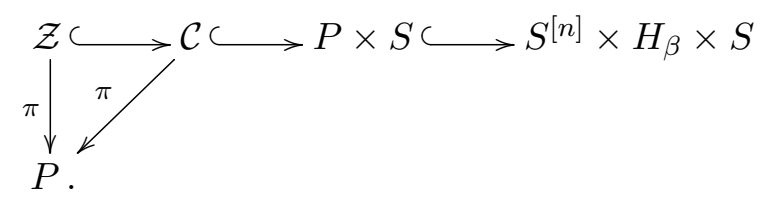

Namely, $\sigma_{\mathcal{C}}=\pi_{*}\left(s_{\mathcal{C}} \mid \mathcal{Z}\right)$, where $s_{\mathcal{C}}$ is the section of $\mathcal{O}(\mathcal{C})$ cutting out $\mathcal{C} \subset S^{[n]} \times H_{\beta} \times S$.

We have the commutative diagram

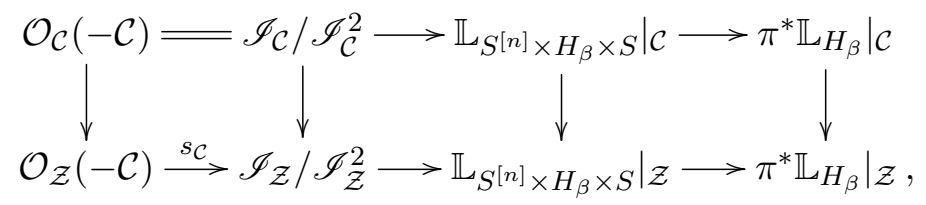

where in the bottom row we have suppressed the pushforward map from $\mathcal{Z}$ to $\mathcal{C}$. Composing horizontally and dualising, by adjunction we get the commutative diagram

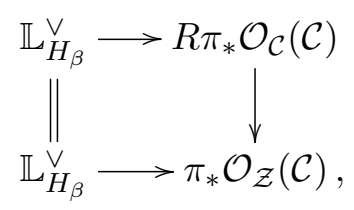

with bottom row induced by $\pi_{*}\left(s_{\mathcal{C}} \mid \mathcal{Z}\right)=\sigma_{\mathcal{C}}$. Dualising this bottom row gives the composition of the second, third and fourth arrows in (98).

Going clockwise round (97) gives

$$
\mathcal{E} \bullet\left(R \pi_{H *} \mathcal{O}_{\mathcal{C}}(\mathcal{C})\right)^{\vee}[1] \longrightarrow \mathbb{L}_{H_{\beta}}[1]
$$

where the second arrow is the dual of (81). But this is the top row of (99), by construction. The first arrow comes from Proposition A.3; the top row of (92) shows it factors through

$$
\mathcal{E}^{\bullet} \longrightarrow\left(\mathcal{O}(\mathcal{C})^{[n]}\right)^{*}[1] \longrightarrow\left(R \pi_{*} \mathcal{O}_{\mathcal{C}}(\mathcal{C})\right)^{\vee}[1]
$$

where the second arrow is the dual of $R \pi_{*} \mathcal{O}_{\mathcal{C}}(\mathcal{C}) \rightarrow R \pi_{*} \mathcal{O}_{\mathcal{Z}}(\mathcal{C})=\mathcal{O}(\mathcal{C})^{[n]}$. But this is the righthand vertical arrow in (99). By the commutativity of (99), then, the two compositions are the same.

It follows that Cone $\left(\mathcal{E} \bullet[-1] \rightarrow F^{\bullet}\right)$ maps to $\mathbb{L}_{P}$ giving a perfect obstruction theory. By Proposition A.3 this perfect obstruction theory is the complex

$$
R \operatorname{Hom}\left(I^{\bullet}, F\right)^{\vee}
$$

at a point $(F, s) \in P$. We have not proven that the map from this complex to $\mathbb{L}_{P}$ is the same as the one given by the perfect obstruction theory of Proposition 3.4. Though they are surely the same, we don't need this to deduce that the resulting virtual cycles are the same since they 


\section{Curve COUnting on SuRfaces I: TheOrY}

depend only on the K-theory class of the two-term complex. What we really want, however, is the same result for the reduced cycles.

The dual semi-regularity map of (79) applied to the exact triangle of Proposition A.3 gives the diagram

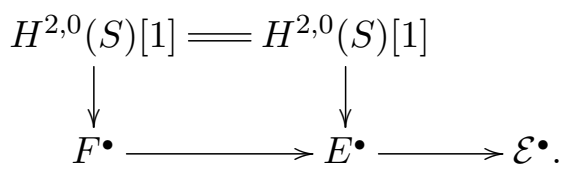

Taking cones gives

$$
F_{\text {red }}^{\bullet} \longrightarrow E_{\text {red }}^{\bullet} \longrightarrow \mathcal{E}^{\bullet}
$$

as desired in (89). Since $F_{r e d}^{\bullet}$ and $\mathcal{E}^{\bullet}$ are perfect two-term complexes, so is $E_{\text {red }}^{\bullet}$.

By Proposition A.2 the map $F^{\bullet} \rightarrow \mathbb{L}_{H_{\beta}}$ (81) descends to $F_{\text {red }}^{\bullet} \rightarrow \mathbb{L}_{H_{\beta}}$ to give the perfect obstruction theory of (77). Therefore by Lemma A.6 the perfect relative obstruction theory $\mathcal{E} \bullet \rightarrow \mathbb{L}_{P / H_{\beta}}$ of (88) fits into a commutative diagram

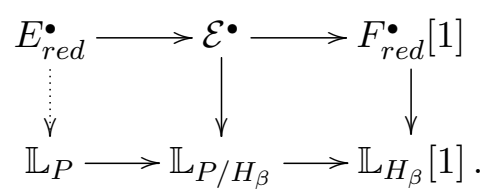

We can fill in the dotted arrow, giving (by the long exact sequence in cohomology) a perfect obstruction theory for $P$. We have not checked that this is the same as the one given by Proposition 3.4, though it surely is. But the complexes $E_{\text {red }}^{\bullet}$ and $\left(\left.E_{X, r e d}^{\bullet}\right|_{P_{S}}\right)^{f i x}$ have the same K-theory classes, since they sit inside exact triangles with the same objects. This is enough to ensure that the reduced virtual cycles are the same.

Theorem A.7. The constructions of this appendix define a reduced virtual cycle

$$
\left[P_{1-h+n}(S, \beta)\right]^{r e d} \in H_{2 v}\left(P_{1-h+n}(S, \beta)\right), \quad v=h-1+n+\int_{\beta} c_{1}(S)+h^{0,2}(S),
$$

whenever condition (2) holds. It coincides with the reduced class (43) when condition (3) also holds. When condition (72) holds, its pushforward to the smooth ambient space $H_{\gamma} \times S^{[n]}$ is

$$
c_{\text {top }}(F) \cdot c_{n}\left(\mathcal{O}\left(\mathcal{D}-\pi^{*} A\right)^{[n]}\right),
$$

where $F$ is the bundle (76).

Proof. Assuming (72), we can summarise the results of this appendix as follows. The perfect obstruction theory $E_{\text {red }}^{\bullet}$ arose from cutting $P$ out of $H_{\gamma} \times S^{[n]}$ firstly by a section $\sigma$ of the vector bundle $F(76)$ (pulled back from $H_{\gamma}$ ) followed by a section $\sigma_{\mathcal{C}}$ of the bundle $\mathcal{O}(\mathcal{C})^{[n]}$ over $H_{\beta} \times S^{[n]}$ (87). The latter bundle extends over $H_{\gamma} \times S^{[n]}$ as the bundle $\mathcal{O}\left(\mathcal{D}-\pi_{S}^{*} A\right)^{[n]}$, even though the section $\sigma_{\mathcal{C}}$ does not. This is enough to give (102). 
The assumption (72) that $H^{2}(L)=0$ for all $L \in \operatorname{Pic}_{\beta}(S)$ was required to ensure that the sheaf $F$ of (76) was a vector bundle on all of $H_{\gamma}$. If instead we impose only condition (2) - that $H^{2}(L)=0$ for all effective $L \in \operatorname{Pic}_{\beta}(S)$ - then $F$ is a sheaf on $H_{\gamma}$ which is locally free on $H_{\beta}$. It is therefore locally free in a neighbourhood of $H_{\beta} \subset H_{\gamma}$.

The global description of $H_{\beta}$ as the zero locus of the section $\sigma$ of $F$ still holds. Since $F$ is a bundle near $H_{\beta}$, this is enough to induce the perfect obstruction theory on $H_{\beta}$. The construction of the reduced class then follows as before.

Since the perfect obstruction theory $E_{\text {red }}^{\bullet}$ has the same K-theory class as $\left(E_{X, r e d}^{\bullet} \mid P_{S}\right)^{f i x}$, when condition (3) also holds we get the same reduced cycle as we do from the perfect obstruction theory of Proposition 3.4.

In [KT14] we use the global description as the zero locus of a section of a bundle to do computations starting from the formula (102). A priori then, these calculations require the stronger hypothesis (72).

\section{REFERENCES}

AIK77 A. Altman, A. Iarrobino and S. Kleiman, Irreducibility of the compactified Jacobian, in Real and complex singularities (Proc. Ninth Nordic Summer School/NAVF Sympos. Math., Oslo, 1976), 1-12, Sijthoff and Noordhoff, Alphen aan den Rijn, 1977.

BeF97 K. Behrend and B. Fantechi, The intrinsic normal cone, Invent. Math. 128 (1997), 45-88. http://dx.doi.org/10.1007/s002220050136

BeF98 K. Behrend and B. Fantechi, Modifying Gromov-Witten invariants for varieties with $h^{2,0} \neq 0$, unfinished manuscript (1998), www.mfo.de/document/9825/Report_24_98.ps

Beh97 K. Behrend, Gromov-Witten invariants in algebraic geometry, Invent. Math. 127 (1997), 601617. http://dx.doi.org/10.1007/s002220050132

BL99 J. Bryan and C. Leung, Generating functions for the number of curves on abelian surfaces, Duke Math. J. 99 (1999), 311-328. http://dx.doi.org/10.1215/S0012-7094-99-09911-8

BL00 J. Bryan and C. Leung, The enumerative geometry of K3 surfaces and modular forms, J. Amer. Math. Soc. 13 (2000), 371-410. http://dx.doi.org/10.1090/ S0894-0347-00-00326-X

Blo72 S. Bloch, Semi-regularity and de Rham cohomology, Invent. Math. 17 (1972), 51-66.

BS91 M. Beltrametti and A. J. Sommese, Zero cycles and $k$ th order embeddings of smooth projective surfaces. With an appendix by Lothar Göttsche, in Problems in the theory of surfaces and their classification (Cortona, 1988), Sympos. Math. 32, 33-48, Academic Press, London, 1991.

BuF03 R.-O. Buchweitz and H. Flenner, A semiregularity map for modules and applications to deformations, Compositio Math. 137 (2003), 135-210. http://dx.doi.org/10.1023/A: 1023999012081

DKO07 M. Dürr, A. Kabanov and C. Okonek, Poincaré invariants, Topology 46 (2007), 225-294. http://dx.doi.org/10.1016/j.top.2007.02.004 


\section{Curve COUNTING ON SURFACES I: THEORY}

Don90 S. K. Donaldson, Yang-Mills invariants of four-manifolds, London Math. Soc. Lecture Note Ser. 150, Cambridge Univ. Press, Cambridge, 1990, 5-40. http://dx.doi.org/10.1017/ CB09780511629334.003

Ful98 W. Fulton, Intersection theory, Second Edition, Ergebnisse der Mathematik und ihrer Grenzgebiete, 3. Folge, Bd. 2, Springer-Verlag, Berlin, 1998. http://dx.doi.org/10.1007/ 978-1-4612-1700-8

Got98 L. Göttsche, A conjectural generating function for numbers of curves on surfaces, Comm. Math. Phys. 196 (1998), 523-533. http://dx.doi.org/10.1007/s002200050434

GP99 T. Graber and R. Pandharipande, Localization of virtual classes, Invent. Math. 135 (1999), 487-518. http://dx.doi.org/10.1007/s002220050293

GV98 R. Gopakumar and C. Vafa, M-theory and topological strings-I, II, arXiv:hep-th/9809187 and arXiv:hep-th/9812127 (1998).

Iar72 A. Iarrobino, Punctual Hilbert schemes, Bull. Amer. Math. Soc. 78 (1972), 819-823.

Ill71 L. Illusie, Complexe cotangent et déformations I, Lecture Notes in Math., vol. 239, SpringerVerlag, Berlin-New York, 1971.

IM13 D. Iacono and M. Manetti, Semiregularity and obstructions of complete intersections, Adv. Math. 235 (2013), 92-125. http://dx.doi.org/10.1016/j.aim.2012.11.011

JS11 D. Joyce and Y. Song, A theory of generalized Donaldson-Thomas invariants, Mem. Amer. Math. Soc. 217 (2012), no. 1020. http://dx.doi.org/10.1090/S0065-9266-2011-00630-1

Kim91 S.-O. Kim, Noether-Lefschetz locus for surfaces, Trans. Amer. Math. Soc. 324 (1991), 369-384. http://dx.doi.org/10.2307/2001513

KL07 Y.-H. Kiem and J. Li, Gromov-Witten invariants of varieties with holomorphic 2-forms, arXiv:0707.2986.

KM76 F. Knudsen and D. Mumford, The projectivity of the moduli space of stable curves I: Preliminaries on "det" and "Div", Math. Scand. 39 (1976), 19-55.

Koo13 M. Kool, Duality and universality for stable pair invariants of surfaces, arXiv:1303.5340.

KS59 K. Kodaira and D. C. Spencer, A theorem of completeness of characteristic systems of complete continuous systems, Amer. J. Math. 81 (1959), 477-500.

KST11 M. Kool, V. Shende and R. P. Thomas, A short proof of the Göttsche conjecture, Geom. Topol. 15 (2011), 397-406. http://dx.doi.org/10.2140/gt.2011.15.397

KT14 M. Kool and R. P. Thomas, Reduced classes and curve counting on surfaces II: calculations, Alg. Geom. 3 (2014), 384-399. http://dx.doi.org/10.14231/AG-2014-018

Lee04 J. Lee, Family Gromov-Witten invariants for Kähler surfaces, Duke Math. J. 123 (2004), 209-233.

Li02 J. Li, A note on enumerating rational curves in a K3 surface, in Geometry and nonlinear partial differential equations (Hangzhou, 2001), 53-62, AMS/IP Studies in Adv. Math. 29, Amer. Math. Soc., Providence, RI, 2002.

Liu00 A.-K. Liu, Family blowup formula, admissible graphs and the enumeration of singular curves, I, J. Differential Geom. 56 (2000), 381-579.

LT98 J. Li and G. Tian, Virtual moduli cycles and Gromov-Witten invariants of algebraic varieties, J. Amer. Math. Soc. 11 (1998), 119-174. 


\section{Kool, R. P. Thomas}

Man07 M. Manetti, Lie description of higher obstructions to deforming submanifolds, Ann. Sc. Norm. Super. Pisa Cl. Sci. 6 (2007), no. 4, 631-659.

MNOP06 D. Maulik, N. Nekrasov, A. Okounkov and R. Pandharipande, Gromov-Witten theory and Donaldson-Thomas theory, I, Compositio Math. 142 (2006), 1263-1285. http://dx.doi.org/ 10.1112/S0010437X06002302

MP13 D. Maulik and R. Pandharipande, Gromov-Witten theory and Noether-Lefschetz theory, in A celebration of algebraic geometry, Clay Math. Proc. 18, Amer. Math. Soc., Providence, RI, 2013, 469-507.

MPT10 D. Maulik, R. Pandharipande and R. P. Thomas, Curves on K3 surfaces and modular forms, J. Topol. 3 (2010), 937-996. http://dx.doi.org/10.1112/jtopol/jtq030

OP10 A. Okounkov and R. Pandharipande, Quantum cohomology of the Hilbert scheme of points in the plane, Invent. Math. 179 (2010), 523-557. http://dx.doi.org/10.1007/ s00222-009-0223-5

Pid91 V. Ya. Pidstrigach, Deformations of instanton surfaces, Izv. Akad. Nauk SSSR Ser. Mat. 55 (1991), 318-338. http://dx.doi.org/10.1070/IM1992v038n02ABEH002201

Pri12 J. P. Pridham, Semiregularity as a consequence of Goodwillie's theorem, arXiv:1208.3111.

PT09 R. Pandharipande and R. P. Thomas, Curve counting via stable pairs in the derived category, Invent. Math. 178 (2009), 407-447. http://dx.doi.org/10.1007/s00222-009-0203-9

PT10 R. Pandharipande and R. P. Thomas, Stable pairs and BPS invariants, J. Amer. Math. Soc. 23 (2010), 267-297. http://dx.doi.org/10.1090/S0894-0347-09-00646-8

PT14 R. Pandharipande and R. P. Thomas, The Katz-Klemm-Vafa conjecture for K3 surfaces, arXiv:1404.6698.

Ran99 Z. Ran, Semiregularity, obstructions and deformations of Hodge classes, Ann. Sc. Norm. Super. Pisa Cl. Sci. 4 (1999), no. 4, 809-820.

Ros12 S. Rose, Counting Hyperelliptic curves on Abelian surfaces with Quasi-modular forms, arXiv:1202.2094.

Sch12 T. Schürg, More Reduced Obstruction Theories, arXiv:1209.4631.

STV13 T. Schürg, B. Toën and G. Vezzosi, Derived algebraic geometry, determinants of perfect complexes, and applications to obstruction theories for maps and complexes, J. Reine Angew. Math., to appear, arXiv:1102.1150 (2011). http://dx.doi.org/10.1515/crelle-2013-0037

Sie04 B. Siebert, Virtual fundamental classes, global normal cones and Fulton's canonical classes, in Frobenius manifolds, eds K. Hertling and M. Marcolli, Aspects Math. 36, Vieweg, Wiesbaden, 2004, 341-358. http://dx.doi.org/10.1007/978-3-322-80236-1_13

Tze12 Y. Tzeng, A proof of the Göttsche-Yau-Zaslow formula, J. Differential Geom. 90 (2012), 439472 .

Voi13 C. Voisin, Hodge loci, in Handbook of moduli: Volume III, eds G. Farkas and I. Morrison, Advanced Lectures in Mathematics 25, Volume III, International Press, 2013, 507-546. 
CuRve COUnTING ON SURFACES I: THEORY

Martijn Kool mkool@math.ubc.ca

Pacific Institute for the Mathematical Sciences, Department of Mathematics, University of British

Columbia, Vancouver, British Columbia, Canada, V6T $1 Z 4$

Richard Thomas richard.thomas@imperial.ac.uk

Department of Mathematics, Imperial College London, London, SW7 2AZ, United Kingdom 